NIST Technical Note 1967

\title{
3.5 GHz Radar Waveform Capture at Fort Story Final Test Report
}

\author{
Paul Hale \\ Jeffrey Jargon \\ Peter Jeavons \\ Michael Souryal \\ Adam Wunderlich \\ Mark Lofquist
}

This publication is available free of charge from:

https://doi.org/10.6028/NIST.TN.1967

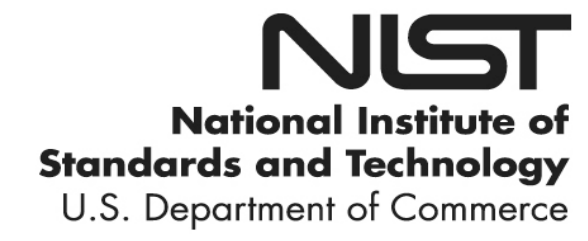




\title{
3.5 GHz Radar Waveform Capture at Fort Story Final Test Report
}

\author{
Paul Hale \\ Jeffrey Jargon \\ Peter Jeavons \\ Michael Souryal \\ Adam Wunderlich \\ Communications Technology Laboratory \\ Mark Lofquist \\ MITRE Corporation \\ Bedford, $M A$
}

This publication is available free of charge from: https://doi.org/10.6028/NIST.TN.1967

October 2017

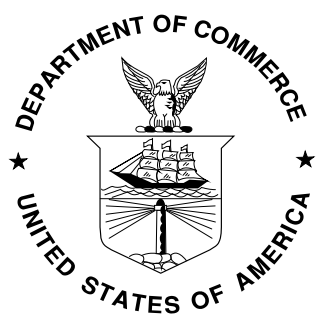

U.S. Department of Commerce Wilbur L. Ross, Jr., Secretary

National Institute of Standards and Technology Walter Copan, NIST Director and Undersecretary of Commerce for Standards and Technology 
Certain commercial entities, equipment, or materials may be identified in this document in order to describe an experimental procedure or concept adequately. Such identification is not intended to imply recommendation or endorsement by the National Institute of Standards and Technology, nor is it intended to imply that the entities, materials, or equipment are necessarily the best available for the purpose.

National Institute of Standards and Technology Technical Note 1967

Natl. Inst. Stand. Technol. Tech. Note 1967, 86 pages (October 2017) CODEN: NTNOEF

This publication is available free of charge from:

https://doi.org/10.6028/NIST.TN.1967

Cleared for Open Publication 24 October 2017 by the Department of Defense Office of Prepublication and Security Review, Reference Number: 17-S-1966 
THIS PAGE INTENTIONALLY LEFT BLANK 
NASCTN Report 3

NIST Technical Note 1967

\title{
3.5 GHz Radar Waveform Capture at Fort Story \\ Final Test Report
}

\author{
Paul Hale, Ph.D., Jeffrey Jargon, Ph.D., Peter Jeavons, \\ Michael Souryal, Ph.D., Adam Wunderlich, Ph.D. \\ Communications Technology Laboratory, NIST \\ Mark Lofquist \\ MITRE Corporation
}

October 30, 2017
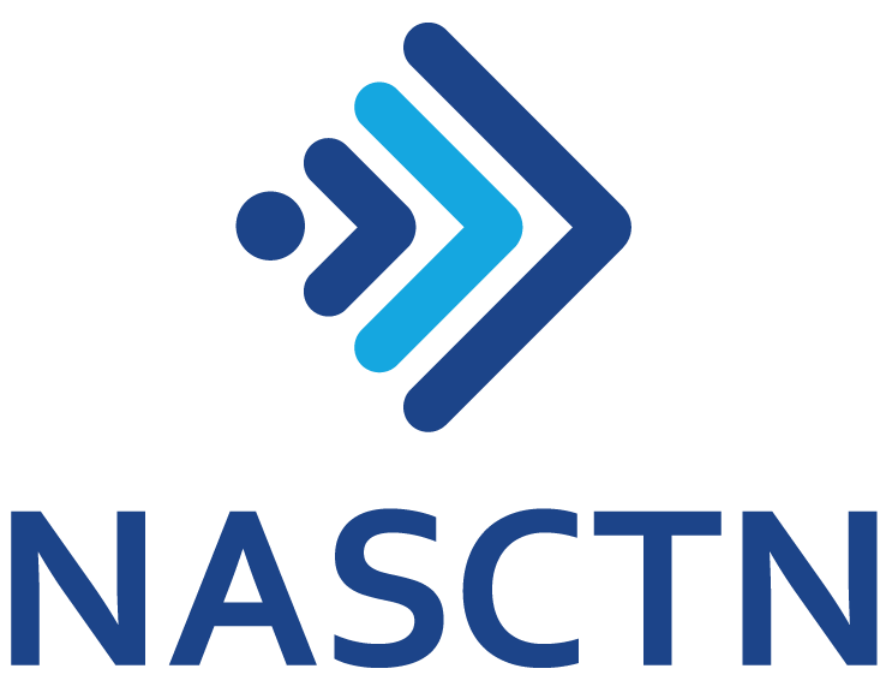

National Advanced Spectrum and Communications Test Network

Cleared for Open Publication 24 October 2017 by the Department of Defense Office of Prepublication and Security Review, Reference Number: 17-S-1966 


\section{National Advanced Spectrum and Communications Test Network (NASCTN)}

The mission of the National Advanced Spectrum and Communications Test Network (NASCTN) is to provide, through its members, robust test processes and validated measurement data necessary to develop, evaluate and deploy spectrum sharing technologies that can increase access to the spectrum by both federal agencies and non-federal spectrum users.

NASCTN was formed to provide a single focal point for engaging industry, academia, and other government agencies on advanced spectrum technologies, including testing, measurement, validation, and conformity assessment. NIST hosts the NASCTN capability at the Department of Commerce Boulder Laboratories in Boulder, Colorado.

NASCTN is a membership organization under a charter agreement. Members

- Make available, in accordance with their organization's rules policies and regulations, engineering capabilities and test facilities, with typical consideration for cost.

- Coordinate their efforts to identify, develop and test spectrum sharing ideas, concepts and technology to support the goal of advancing more efficient and effective spectrum sharing.

- Make available information related to spectrum sharing, considering requirements for the protection of intellectual property, national security, and other organizational controls, and, to the maximum extent possible, allow the publication of NASCTN test results.

- Ensure all spectrum sharing efforts are identified to other interested members.

Current charter members are:

- National Telecommunications and Information Administration (NTIA)

- National Institute of Standards and Technology (NIST)

- Department of Defense Chief Information Officer (DoD CIO) 


\title{
Acknowledgments
}

\begin{abstract}
We thank the Department of Defense and the Shipboard Electronics Systems Evaluation Facility (SESEF) located at Fort Story, Virginia Beach, Virginia, for providing access to the measurement site; Michael Cotton and his colleagues at the National Telecommunications and Information Administration (NTIA), Institute for Telecommunications Sciences (ITS), Boulder, Colorado, for the use of the pre-selector; Frank Sanders at NTIA/ITS for sharing his expertise on radar systems and measurements; John Ladbury at NIST for characterizing the antennas we used in our measurements; and Sheryl Genco, NASCTN program manager, for stakeholder management, project management, and coordination.
\end{abstract}




\section{Executive Summary}

In view of recently adopted Federal Communications Commission (FCC) rules to share the spectrum from $3550 \mathrm{MHz}$ to $3700 \mathrm{MHz}$ between federal and commercial users, NASCTN launched a measurement effort to create a library of high-fidelity waveforms of federal incumbent radar signals currently operating in the $3550 \mathrm{MHz}$ to $3650 \mathrm{MHz}$ ("3.5 GHz") band. The purpose of this waveform library is to facilitate the development and testing of a commercial environmental sensing capability (ESC) that will detect federal incumbent systems and permit spectrum sharing in U.S. coastal exclusion zones, in accordance with FCC rules.

The primary federal incumbent in the $3.5 \mathrm{GHz}$ band is the U.S. Navy shipborne SPN-43 air traffic control radar, as well as other Department of Defense (DoD) ground-based radar systems. Since the focus of this study was on the shipborne radar, measurements were conducted at two coastal locations, Point Loma, near San Diego, California, and Fort Story, near Virginia Beach, Virginia. This report documents the methodology and results of the measurements collected at Fort Story; the Point Loma measurements were documented in a prior report [1].

Measurements were collected at Fort Story over a two-month period. They consist of 60-second power spectral measurements collected every 10 minutes as well as high-resolution in-phase and quadrature (IQ) digital waveforms covering the entire $3.5 \mathrm{GHz}$ band and portions of adjacent bands (3477 MHz to $3677 \mathrm{MHz}$ ). The digital waveforms were retained if energy was detected above a predefined threshold in the band of interest. A directional, cavity-backed spiral antenna was used during the first month of data collection, after which an omnidirectional, slant-polarized antenna was used.

The Fort Story measurement campaign resulted in a total of 617960 -s power spectra and 1473 high-resolution 60 -s waveforms. Over the course of these measurements, the target SPN43 radar system was observed at three different operational frequencies: $3570 \mathrm{MHz}, 3600 \mathrm{MHz}$, and $3630 \mathrm{MHz}$. In addition to observing SPN-43 at these frequencies in "normal" operation, the following phenomena were observed and are documented in this report:

- Tuning of the radar across a contiguous range of frequencies 
- Adjacent-band emissions into the band of interest

- Simultaneous emission from two SPN-43 systems in two different frequency channels

Furthermore, out-of-band emissions from adjacent-band systems were frequently observed to be coincident and co-channel with SPN-43 emissions, indicating that the adjacent-band emissions could confound SPN-43 detection by an ESC.

A subset of the high-resolution IQ waveforms containing no adjacent-band or other in-band emissions was selected for further statistical analysis. This analysis focused on key parameters of the target radar that could aid detection, namely, the antenna rotation period, pulse repetition interval, pulse duration, and center frequency. The subset of approximately 250 "clean" acquisitions of in-band radar consisted of 2700 sweeps of the main beam and 48600 individual pulses ranging in peak power by over $40 \mathrm{~dB}$. The statistical analysis of in-band radar parameters yielded the following observations.

- The median antenna rotation period was $3.86 \mathrm{~s}, 0.14 \mathrm{~s}$ lower than reported in the SPN43 system manual dated 2005 [2]. The difference between the 10th and 90th percentiles (containing $80 \%$ of the observations) was $0.06 \mathrm{~s}$.

- The median pulse repetition interval was very close to the nominal value of $1 \mathrm{~ms}$ (only $13 \mathrm{~ns}$ greater). Furthermore, the difference between the 1st and 99th percentiles (containing $98 \%$ of the values) was only $53 \mathrm{~ns}$.

- The overall median pulse duration was $1.15 \mu \mathrm{s}$ and varied by $0.05 \mu \mathrm{s}$ across the three observed frequencies. The 1st and 99th percentiles of the pulse duration were $1.05 \mu \mathrm{s}$ and $1.17 \mu \mathrm{s}$, respectively. The median pulse duration observed here is $0.20 \mu \mathrm{s}$ longer than that reported in the SPN-43 system manual [2].

- The median frequency offset from the nominal center frequency ranged from $174 \mathrm{kHz}$ to $745 \mathrm{kHz}$ across the three observed frequencies, and their $98 \%$ spreads varied from $275 \mathrm{kHz}$ to $1.07 \mathrm{MHz}$.

Knowledge of these statistics can be used to develop robust ESC detectors. They can also inform the development and selection of waveforms with which these detectors can be tested by a certification body. Furthermore, the waveforms themselves could be "played back" - that is, converted to an analog signal and upconverted to the appropriate radio frequency (RF) - by a vector signal generator to evaluate the performance of an ESC detector. A benefit of playing back the measured waveforms is that the effects of the propagation channel, which can distort the signal, are inherent in these waveforms. Hence, the measured waveforms better reflect the actual signals an ESC will receive in the field.

While the focus of this study was on currently-deployed shipborne radars in the band of interest, an important consideration in future work is the class of more sophisticated radars yet to be 
deployed in this band and which will also need to be detected by commercial users and protected from harmful interference. It is unlikely that the waveforms of these future systems will be shared with commercial users for development of their detectors, hence there is a need to develop and validate surrogate waveforms that adequately represent them.

Another area of interest for future research is the development and validation of $3.5 \mathrm{GHz}$ channel models. These models would be of use to commercial developers of Spectrum Access Systems for $3.5 \mathrm{GHz}$, which must predict and manage aggregate interference to federal and other incumbents. Furthermore, wideband channel impulse response models, that include the effects of a multipath channel on the pulse shape, would have value in the testing of ESC detectors. They could be used in conjunction with surrogate radar waveforms to render them more representative of the signals that ESC detectors will observe in the field. 


\section{Contents}

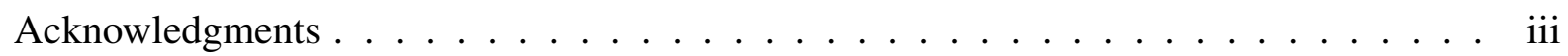

Executive Summary . . . . . . . . . . . . . . . . . . . v

List of Acronyms . . . . . . . . . . . . . . . . . xi

List of Figures . . . . . . . . . . . . . . . . . . xii

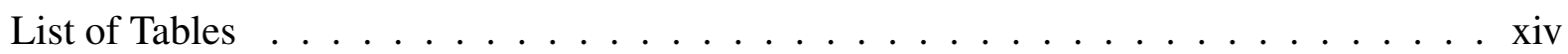

1 Introduction $\quad 1$

2 Test Summary $\quad 5$

2.1 Measurement configuration ...................... 5

2.1 .1 Location . . . . . . . . . . . . . . . . . 5

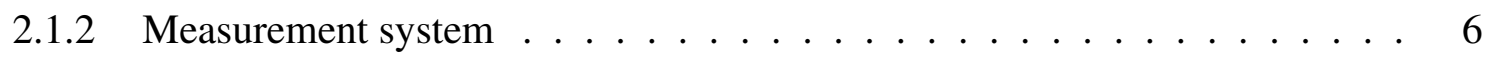

2.2 Statistical considerations . . . . . . . . . . . . . . . . . 10

2.2.1 Blind sampling . . . . . . . . . . . . . . . . . 10

2.2.2 Relevant variables and potential sampling biases . . . . . . . . . . . . 10

2.2.3 Trigger and data retention criteria . . . . . . . . . . . . . 11

2.3 Measurement calibration . . . . . . . . . . . . . . . 13

2.3.1 RF section magnitude response . . . . . . . . . . . . . . . . 14

2.3.2 RF section group delay . . . . . . . . . . . . . . . . . . . . . . . . . . 15

2.3.3 VST response magnitude . . . . . . . . . . . . . . . 15

2.3.4 VST local oscillator and sampling frequencies . . . . . . . . . . . . 16

2.3.5 Antenna characterization . . . . . . . . . . . . . . 17

2.3 .6 System noise floor ... . . . . . . . . . . . . . . . . . . . . . . . . . . . 19

2.3 .7 System nonlinearity . . . . . . . . . . . . . . . . . . . . 19

2.3.8 Summary and discussion of system gain and uncertainty . . . . . . . . 20

3 Qualitative Results $\quad 29$

3.1 Overview of SPN-43 waveforms . . . . . . . . . . . . . . . . . 29

3.2 Spectrograms and their projections onto the time and frequency axes . . . . . . 30

3.3 Qualitative behavior of SPN-43 in normal operation . . . . . . . . . . . . . 33

3.4 Observed SPN-43 anomalous behavior . . . . . . . . . . . . . . . . . . 34

3.5 Adjacent-band emitters . . . . . . . . . . . . . . . . 34 
4 Waveform Parameter Analysis 49

4.1 Summary of collected data and processing . . . . . . . . . . . . . . . . . . . . . . . . 49

4.2 Sweep parameters . . . . . . . . . . . . . . . . . . 50

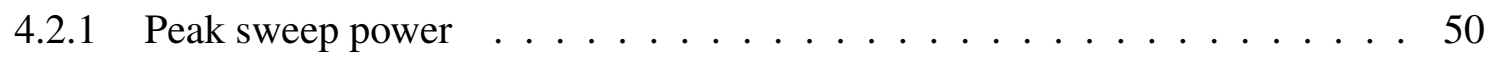

4.2 .2 Sweep interval . . . . . . . . . . . . . . . 51

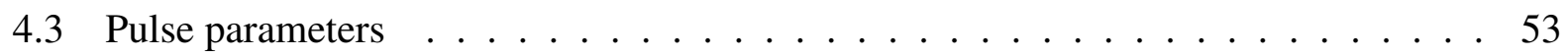

4.3.1 Pulse duration . . . . . . . . . . . . . . . . 53

4.3 .2 Pulse repetition interval . . . . . . . . . . . . . . 56

4.3 .3 Frequency offset . . . . . . . . . . . . . . 58

4.4 Summary statistics . . . . . . . . . . . . . . . . 60

5 Conclusions $\quad 63$

6 Areas for Future Research

$\begin{array}{lr}\text { Bibliography } & 67\end{array}$ 


\section{Acronyms}
ADC analog-to-digital converter
BPF bandpass filter
CBRS Citizens Broadband Radio Service
CBS cavity-back spiral
CDF cumulative distribution function
CCDF complementary cumulative distribution function
DoD Department of Defense
ESC environmental sensing capability
FCC Federal Communications Commission
FFT fast Fourier transform
GbE gigabit ethernet
IQ in-phase and quadrature
ITS Institute for Telecommunication Sciences
LNA low-noise amplifier
LTE long term evolution
NASCTN National Advanced Spectrum and Communications Test Network
NEP noise equivalent power
NIST National Institute of Standards and Technology
NTIA National Telecommunications and Information Administration
OSLT open-short-load-thru
RAID redundant array of independent disks
RF radio frequency
SFDR spur-free dynamic range
SNR signal-to-noise ratio
VNA vector network analyzer
VST vector signal transceiver 


\section{List of Figures}

2.1 View of Fort Story measurement site from $75.3 \mathrm{~km}$ altitude $\ldots \ldots \ldots$

2.2 View of Fort Story measurement site from 626 m altitude $\ldots \ldots \ldots$. . . . . . . . 7

2.3 Detail of rooftop measurement site showing equipment deployment $\ldots \ldots \ldots$

2.4 Schematic diagram of measurement system . . . . . . . . . . . . . . . . . 9

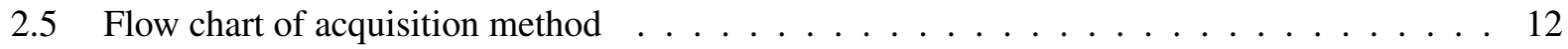

2.6 Transmission measurements of the pre-selector's original $3.5 \mathrm{GHz}$ path . . . . . . . . 21

2.7 Transmission measurements of the pre-selector's original $3.5 \mathrm{GHz}$ path low-frequency

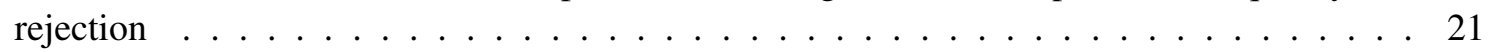

2.8 Transmission measurements of the pre-selector's original $3.5 \mathrm{GHz}$ path high-frequency

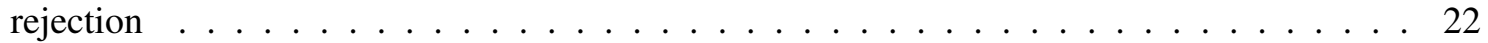

2.9 Transmission measurements of the old and new pre-selector's $3.5 \mathrm{GHz}$ path . . . . . . . . 22

2.10 Transmission measurements of the old and new pre-selector's $3.5 \mathrm{GHz}$ path (zoomed in) . . 23

2.11 Transmission measurements of the long cable . . . . . . . . . . . 23

2.12 Transmission measurement of the short cable . . . . . . . . . . . . . . . . 24

2.13 Normalized response of the VST measured at room temperature . . . . . . . . . . . 25

2.14 Normalized out-of-band rejection of pre-selector plus VST $\ldots \ldots \ldots$

2.15 Normalized out-of-band rejection of new pre-selector plus VST . . . . . . . . . . . . . 27

2.16 CBS antenna pattern at three frequencies . . . . . . . . . . . . . 28

3.1 Magnitude of an IQ capture of a typical $3570 \mathrm{MHz}$ SPN-43 radar . . . . . . . . . . . . 30

3.2 A 1 s duration sub-epoch of the SPN-43 radar capture . . . . . . . . . . . . . . 31

3.3 A 100 ms duration sub-epoch of the SPN-43 radar capture . . . . . . . . . . . . . 31

3.4 A $4 \mu$ s duration sub-epoch of the SPN-43 radar capture . . . . . . . . . . . . . . . . . 32

3.5 Spectrogram (colored surface plot) assembled from spectral metadata . . . . . . . . . . . 36

3.6 Spectrogram of a SPN-43 signal with asymmetric power spectrum . . . . . . . . . . . . 37

3.7 Spectrogram of a SPN-43 signal with temporal fading $\ldots \ldots \ldots \ldots$

3.8 Spectrogram of SPN-43 signals at $3600 \mathrm{MHz}$ and $3630 \mathrm{MHz} \ldots \ldots \ldots$. . . . . . . . . . 39

3.9 Spectrogram of SPN-43 signals at $3570 \mathrm{MHz}$ and $3600 \mathrm{MHz} \ldots \ldots$. . . . . . . . . . . . . 40

3.10 Spectrogram of a SPN-43 signal being tuned to a different frequency . . . . . . . . . . 41

3.11 Apparent capture of SPN-43 turning on and off . . . . . . . . . . . . . . . . . . . 42

3.12 Apparent capture of SPN-43 turning off and then on off $\ldots \ldots \ldots$. . . . . . . . 43

3.13 Broadband emissions of Radar 3 into $3550 \mathrm{MHz}$ to $3650 \mathrm{MHz}$ band . . . . . . . . . . . . . 44

3.14 Spectrogram showing emissions from Radar 3 signal at frequencies below $3520 \mathrm{MHz}$ and broadband pedestal . . . . . . . . . . . . . . . . . 45

3.15 Spectrogram showing emissions from Radar 3 with aliased signal above $3670 \mathrm{MHz}$. . . 46

3.16 Spectrogram showing broadband noise from Radar 3 obscuring SPN-43 signal $\ldots \ldots$. . . 47

4.1 Histogram of peak power per antenna sweep . . . . . . . . . . . . . . 51 
4.2 Empirical CDF and complementary CDF (CCDF) of sweep interval . . . . . . . . . . . 52

4.3 Empirical CDF and CCDF of sweep interval by center frequency $\ldots \ldots \ldots$

4.4 Empirical CDF and CCDF of pulse duration over all data . . . . . . . . . . . . . . . . 54

4.5 Empirical CDF and CCDF of pulse duration by center frequency . . . . . . . . . . . . . . 54

4.6 Empirical CDF and CCDF of pulse duration by antenna type . . . . . . . . . . . . 55

4.7 Empirical CDF and CCDF of pulse duration at $3630 \mathrm{MHz}$ by antenna type $\ldots \ldots 55$

4.8 Empirical CDF and CCDF of pulse repetition interval over all data . . . . . . . . . . . 56

4.9 Empirical CDF and CCDF of pulse repetition interval by antenna type and by frequency . . 57

4.10 Empirical CDF and CCDF of frequency offset over all data $\ldots \ldots \ldots$

4.11 Empirical CDF and CCDF of frequency offset by center frequency . . . . . . . . . . 59

4.12 Histogram of frequency offset by center frequency . . . . . . . . . . . . . . . . 59 


\section{List of Tables}

1.1 Relevant parameters of SPN-43 radar $\ldots \ldots \ldots \ldots \ldots$

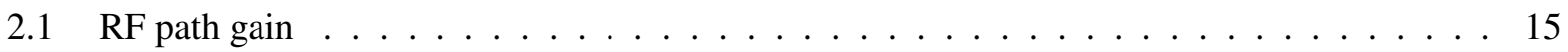

2.2 Estimated gain of omni-directional antenna $\ldots \ldots \ldots \ldots \ldots$

2.3 Boresight gain of CBS antenna at three frequencies . . . . . . . . . . . . . . . . 19

2.4 System noise equivalent power . . . . . . . . . . . . . . . . . . . . . . . . . 19

2.5 Summary of component gains . . . . . . . . . . . . . . . . . 20

4.1 Percentage of processed data by antenna and pulse center frequency . . . . . . . . . . . 50

4.2 Number of days over which data was collected . . . . . . . . . . . . . . . . . 50

4.3 Statistics of sweep and pulse parameters by antenna . . . . . . . . . . . . 61

4.4 Statistics of sweep and pulse parameters by center frequency . . . . . . . . . . . . . . 61 


\section{Chapter 1}

\section{Introduction}

FCC rules issued in April 2015 for the Citizens Band Radio Service (CBRS) permit commercial users to offer wireless broadband services in the $3550 \mathrm{MHz}$ to $3700 \mathrm{MHz}$ band currently occupied by federal and non-federal incumbents [3, 4]. As part of the CBRS architecture, an environmental sensing capability (ESC) will facilitate the coexistence of CBRS users with federal incumbent users through signal sensing and detection of the incumbent signal, permitting CBRS devices to be assigned non-interfering channels in otherwise excluded areas. The federal incumbents in this band include U.S. Navy radar systems operating between $3550 \mathrm{MHz}$ and $3650 \mathrm{MHz}$ (referred to in this report as the "3.5 GHz" band). There is a need for measurements and methodologies with which to test and evaluate the ability of an ESC detector to detect the presence of a signal from a federal system in and adjacent to the $3.5 \mathrm{GHz}$ band. More specifically, there is a need for representative waveforms of federal incumbent signals with which to design and test the ESC detectors.

In this document, we describe the second phase of an effort to create a library of high-fidelity $3.5 \mathrm{GHz}$ federal incumbent radar waveforms that can be used by CBRS ESC providers, federal regulators, and the DoD for ESC design, training, and testing. In the first phase, we measured $3.5 \mathrm{GHz}$ band waveforms at Point Loma, near San Diego, California, and documented the results in [1]. The second phase of measurements, documented in this report, was conducted at Fort Story, near Virginia Beach, Virginia. Together, the library contains measured waveforms that are representative of the waveforms that ESCs will be required to detect, particularly targeting SPN-43 emissions between $3550 \mathrm{MHz}$ and $3650 \mathrm{MHz}$. Recording actual emissions allows for both qualitative and quantitative characterization of Navy system emissions that have only loosely been described in the open literature. In order to quantify variations in the waveforms that will be received by the ESCs due to the differences in the deployed radar equipment and due to the effects of dispersion, multipath fading, and loss in the propagation channel, the library contains measurements of SPN-43 signals emitted from a diversity of littoral sources and power levels. We also collected measurements with two different antennas to increase the diversity of channels measured. 


\begin{tabular}{|lcc|}
\hline Transmitter & 1973 Specification [5, 6] & 2005 Specification [2] \\
\hline Tuning range $(\mathrm{MHz})$ & 3590 to 3700 & 3500 to 3700 \\
Pulse generation method & Magnetron & Magnetron \\
Pulse repetition interval $(\mu \mathrm{s})$ & $889( \pm 25)$ & 1000 \\
Pulse duration $(\mu \mathrm{s})$ & $0.9( \pm 0.15)$ & $0.95( \pm 0.05)$ \\
Peak power output $(\mathrm{kW})$ & $850( \pm 150)$ & 1000 \\
\hline Antenna & & \\
\hline Polarization & Horizontal or left-hand circular, switchable \\
Gain (boresight, $\mathrm{dBi})$ & 32 & $>33.4$ \\
Rotation period $(\mathrm{s})$ & 4 & 4 \\
\hline
\end{tabular}

Table 1.1: Relevant parameters of SPN-43 radar from the most current 2005 specification [2], which differs somewhat from the 1973 specification in [5] and [6], particularly in pulse repetition interval, peak power, and antenna gain.

Operating parameters of the SPN-43 radar system have not been readily available to the public. Some generic specifications of SPN-43 were listed in [5] (which were also repeated in [6]). Updated specifications are given in [2] and are listed in Table 1.1. A description of SPN-43 capabilities and how the system is used operationally is given in [7]. NTIA has had previous projects concerned with measuring various aspects of SPN-43 emissions. High dynamic range measurements of the spectra of SPN-43 emissions at a ground-based site were previously reported in [8]. Spectra, however, do not fully describe the individual radar pulses or beam sweep because they do not include phase information, and so cannot be used to reconstruct the signal waveforms for testing purposes. A small number of IQ waveforms were also recorded for that effort but only characterize the operation of a particular source with distortion due to that particular over-land channel, and the number of recorded waveforms are not adequate to develop a statistical characterization of the radar signals. Reference [6] reports representative time-domain recordings of the SPN-43 antenna rotation, pulse repetition interval, and pulse shape, but the number and diversity of recorded waveforms are again not adequate for a statistical characterization.

In Chapter 2 we describe the measurement equipment and methodology we used to assemble a library of digitally sampled and stored waveforms emitted by SPN-43 and other systems in the $3.5 \mathrm{GHz}$ band. The data collected at Fort Story is comprised of 1473 waveforms, of about $60 \mathrm{~s}$ duration and $200 \mathrm{MHz}$ bandwidth, recorded after down-conversion and sampling of the in-phase and quadrature (IQ) components. These waveforms give detailed information on the radar pulses and can be used to synthesize or "play back" realistic signals for ESC training or testing. The data also includes 6179 metadata files with aggregated, low-resolution, spectrograms and aggregated, low-resolution, time-domain data. In Chapter 3, we use this metadata to describe the qualitative behavior and usage of SPN-43 as well as emissions into the $3.5 \mathrm{GHz}$ band from adjacent-band occupants. Chapter 4 summarizes statistics of the sweep and pulse parameters of a high signal-tonoise ratio (SNR) subset of the IQ waveforms. This subset includes 250 waveforms, 2700 sweeps 
of the radar over our location, and 48600 individual pulses. In Chapter 5 we draw conclusions on the work, and in Chapter 6 we describe future work that could build on this study. 


\section{Chapter 2}

\section{Test Summary}

\subsection{Measurement configuration}

The primary goal of this effort was to collect high fidelity measurements of radar emissions in the $3.5 \mathrm{GHz}$ band at Fort Story. After initial setup and validation, we acquired measurements over a period of two months with the objective of capturing the emissions of a number of SPN-43 systems. To facilitate future testing of ESCs, we recorded waveforms that could be played back in real time. We determined, from industry requirements being developed [9], that we needed to record waveforms with at least $60 \mathrm{~s}$ duration. With strong emissions from Radar 3 in the adjacent $3100 \mathrm{MHz}$ to $3500 \mathrm{MHz}$ band [8, 10], we also wanted to be able to investigate signals in the 3.5 GHz band conditioned on occupancy of the $3100 \mathrm{MHz}$ to $3500 \mathrm{MHz}$ band.

\subsubsection{Location}

The location for data collection met the following criteria: known SPN-43 activity, clear lineof-sight view of the ocean over approximately $180^{\circ}$ azimuth, and data and equipment security. Arranged in coordination with the DoD, the location used for the measurement campaign reported here is at the U.S. Navy Shipboard Electronics Systems Evaluation Facility (SESEF) at Fort Story in Virginia Beach, VA, specifically on the roof of a small building at $36^{\circ} 55^{\prime} 4.88^{\prime \prime} \mathrm{N}, 75^{\circ} 59^{\prime}$ 44.50" W (see Figs. 2.1-2.3). 


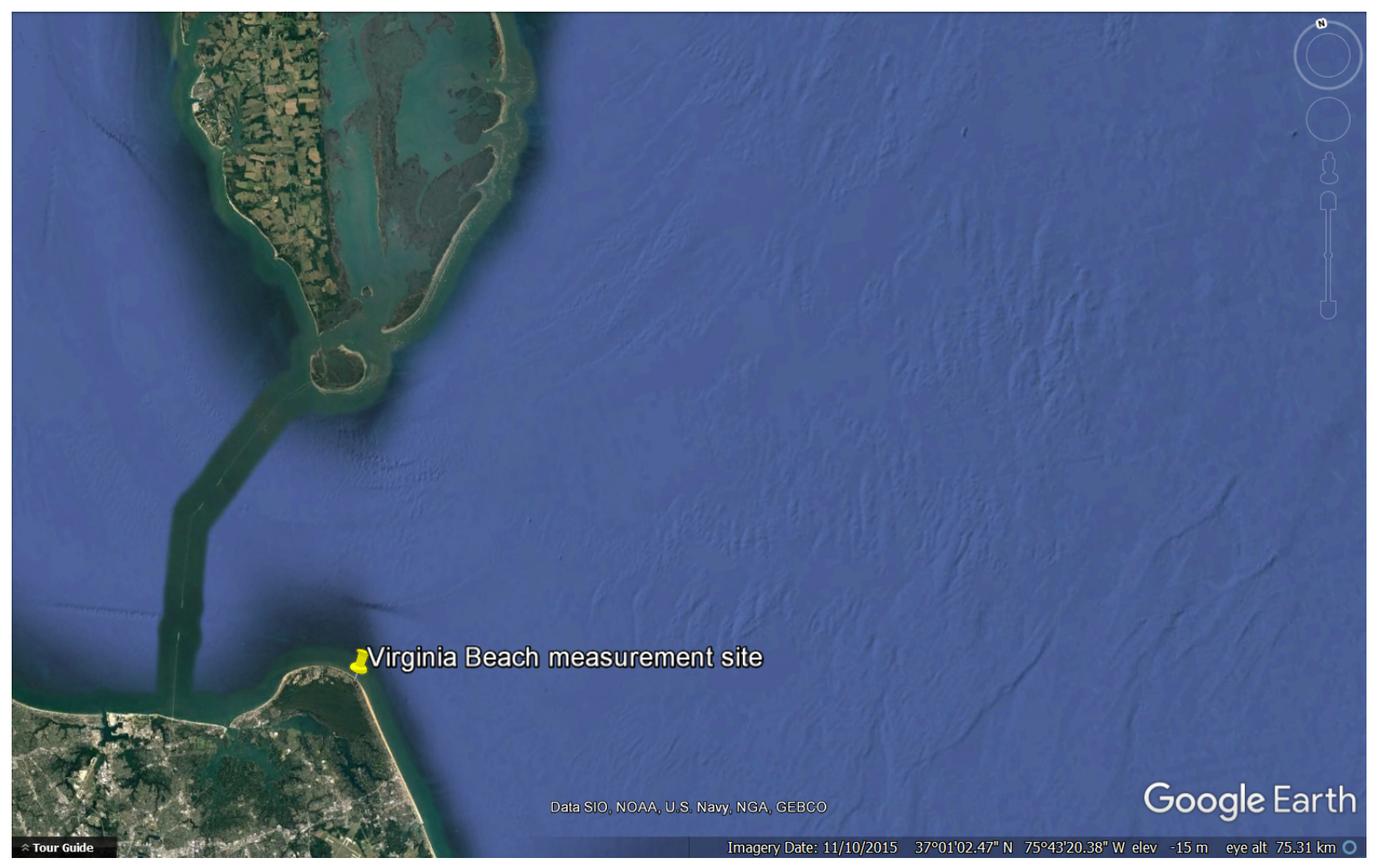

Figure 2.1: View of Fort Story measurement site from $75.3 \mathrm{~km}$ altitude.

\subsubsection{Measurement system}

The equipment used for this measurement campaign is illustrated in Fig. 2.4, and included:

- two antennas (used separately) for capturing the emissions of SPN-43 systems

- a short cable from the output of the antenna to the input of the pre-selector, which consisted of a bandpass filter (BPF) and a low-noise amplifier (LNA) contained in a shielded box, with filtered power supply

- a long cable from the output of the pre-selector to the input of the receiver

- the receiver, which consisted of a Vector Signal Transceiver (VST) in conjunction with a controller

- two pairs of RAID (redundant array of independent disks) drives (one pair for storage, and the other for backup)

- an LTE (long term evolution) router for remote access and control of the system

- an LTE antenna 


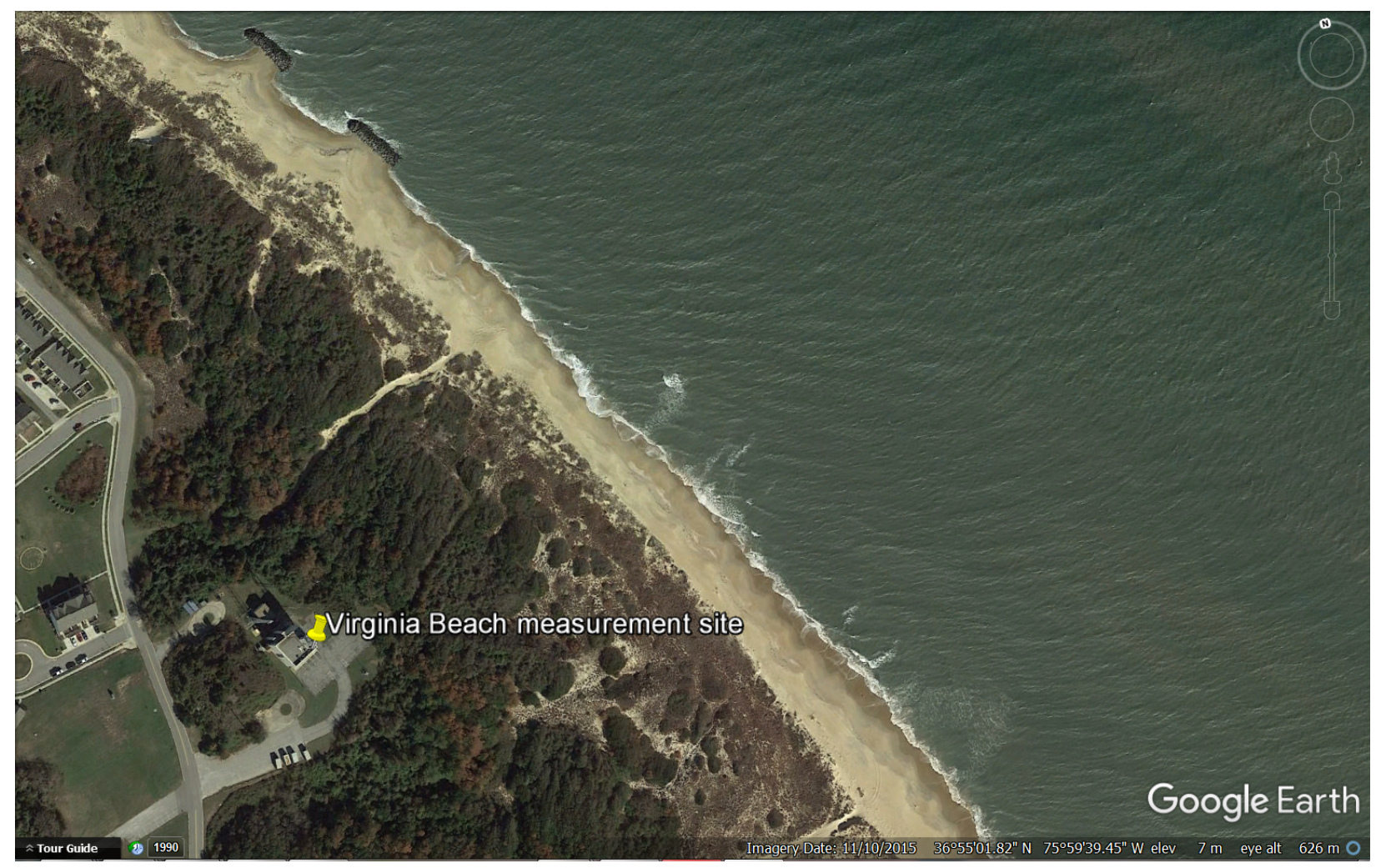

Figure 2.2: View of Fort Story measurement site from $626 \mathrm{~m}$ altitude. The measurement building is indicated with a yellow pin.

We used two different types of antennas for capturing SPN-43 emissions to add to the diversity of propagation channels. A cavity-backed spiral (CBS) antenna was placed in the system for the first half of the measurement campaign, and a slant-polarization, omnidirectional antenna was placed in the system for the second half. The CBS antenna was pointed due east with zero elevation angle. The short cable connecting the output of the antenna to the pre-selector was low-loss (approximately $1 \mathrm{~dB}$ of insertion loss) and included Type- $\mathrm{N}$ connectors.

The pre-selector was constructed by the Institute for Telecommunication Sciences (ITS), the research and engineering arm of the National Telecommunications and Information Administration (NTIA). The pre-selector (Serial Number PS4) was capable of switching between $3.0 \mathrm{GHz}$ and $3.5 \mathrm{GHz}$ bandpass filters; we made use of the $3.5 \mathrm{GHz}$ bandpass filter in series with the LNA for signal selection and amplification purposes. When we switched from the CBS antenna to the omnidirectional antenna, we also replaced the $3.5 \mathrm{GHz}$ bandpass filter with a similar one with a narrower passband to achieve better rejection of adjacent-band emissions. We refer to this filter as the 'new' filter. Since both filters had nearly identical values of insertion loss in the passband, the overall gain of the pre-selector remained constant, and was approximately $29.1 \mathrm{~dB}$. 


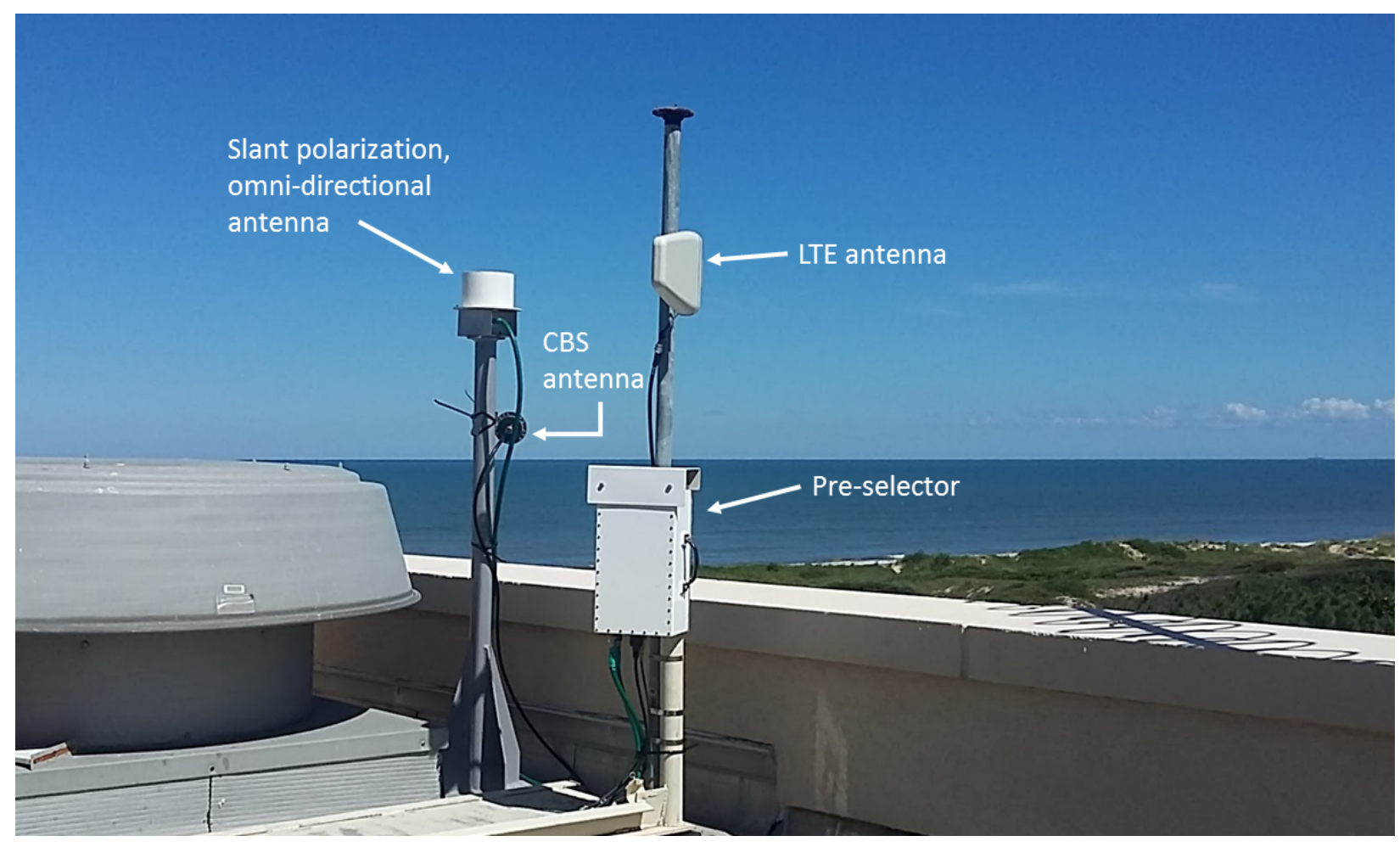

Figure 2.3: Detail of rooftop measurement site showing equipment deployment, including cavitybacked spiral (CBS) antenna and omni-directional antenna. Antenna height above sea level is approximately $20 \mathrm{~m}$.

Since the pre-selector was located on the roof of the building near the antenna, a long cable was required to connect to the input of the VST, which was located inside an enclosed area also located on the roof, along with the RAID drives and the LTE router. This long cable had approximately $5.85 \mathrm{~dB}$ of insertion loss and included Type-N connectors.

The output of the pre-selector was fed into the receiver, a National Instruments (NI) PXIe5646R VST. ${ }^{1}$ The VST uses a direct down conversion technique, with the LO frequency $f_{L O}$ centered in the band of interest. This means that signal frequencies $f_{s}=f_{L O} \pm \Delta$ are down converted to $\pm \Delta$. The output of the down converter is then fed to an IQ demodulator, whose quadrature outputs are sampled (synchronously) with ADCs, each supporting a sample rate of up to $250 \mathrm{MSamples} / \mathrm{s}$ and a sample resolution of 14 bits. The system frequency range is specified to be between $65 \mathrm{MHz}$ and $6.0 \mathrm{GHz}$, with a bandwidth of $200 \mathrm{MHz}\left( \pm 100 \mathrm{MHz}\right.$ around $\left.f_{L O}\right)$. The VST was contained in an NI PXIe-1085 chassis along with an NI PXIe-8880 embedded controller. Our data collection software, written in LabVIEW code, ran on the controller, and included useradjustable settings for the VST reference level, sample rate, file-naming scheme, and threshold

\footnotetext{
${ }^{1}$ Use of trade names in this document is solely for the description of the experimental setup and does not constitute endorsement by NIST or CTL. Other instruments may perform as well or better for this application.
} 


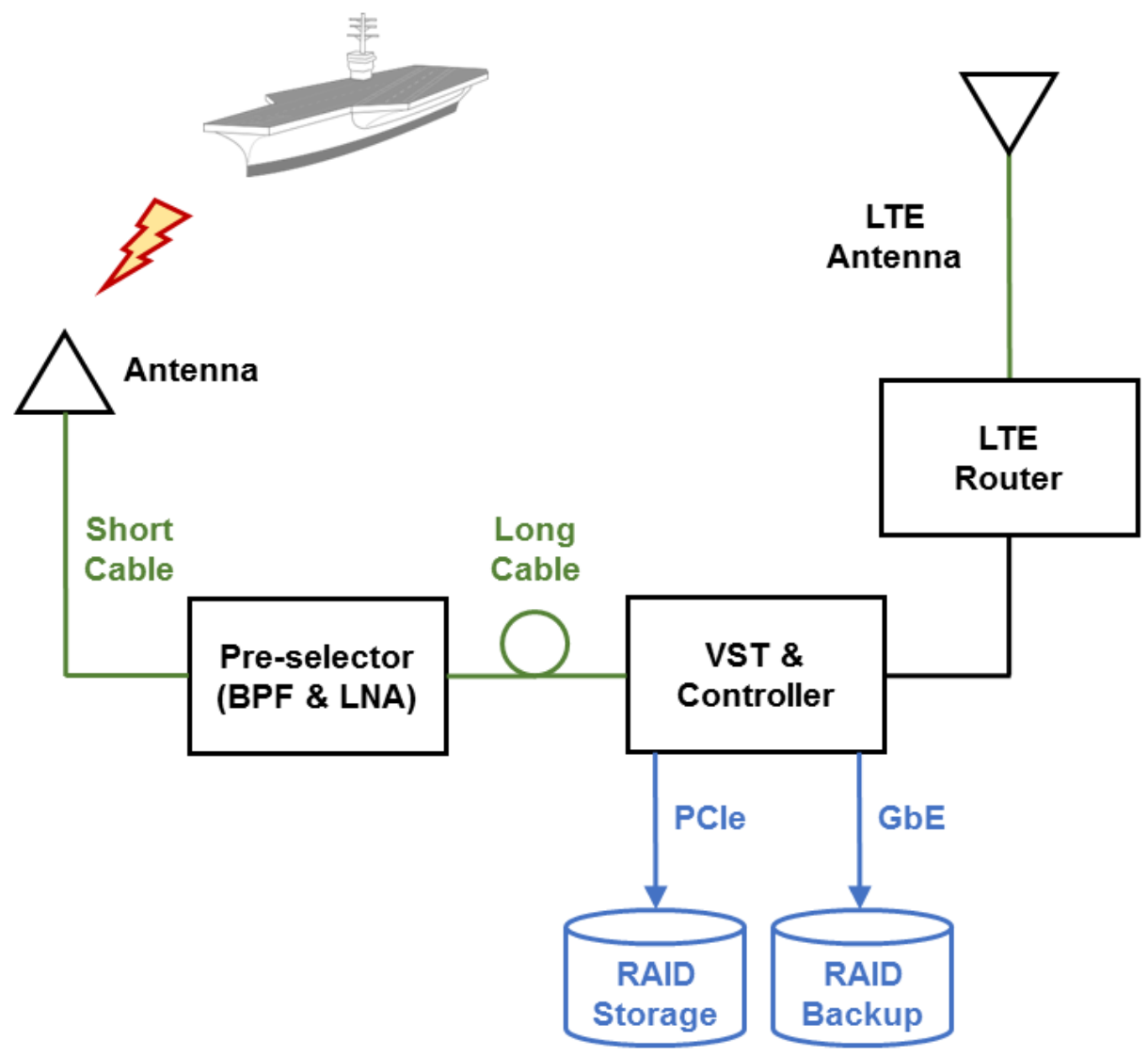

Figure 2.4: Schematic diagram of measurement system

level for determining whether or not to save the captured data.

The data sampled by the VST were stored on a pair of 24-TB RAID drives (referred to later as fast RAIDs) connected to the PXIe chassis via PCIe cables. An additional pair of 24-TB RAID drives (referred to later as slow RAIDs) with gigabit Ethernet (GbE) cables were used to store the data for back-up purposes. The PXIe chassis was also connected to an LTE router along with an LTE antenna to enable remote access. 


\subsection{Statistical considerations}

\subsubsection{Blind sampling}

Our sampling was not coordinated with the Navy; we did not have prior knowledge of the location of the signal source or of the time and frequency of transmission, nor did we obtain ship locations or assigned frequencies during or after the measurements. Thus, our sampling was necessarily blind, and we did not target acquisition of any particular source or time window.

We did not know if emissions observed at different frequencies and/or times were from the same or different shipborne radars. We initially conjectured that, due to the DoD need to coordinate spectrum usage, each frequency observed would correspond to a unique source, and conversely, each source would correspond to a unique frequency. That is, if we acquired SPN-43 signals at two different frequencies, we could be reasonably certain that the signals were emanating from two distinctly different sources. Since our goal was to acquire signals from at least three different sources, we addressed our lack of knowledge by acquiring data until we measured SPN-43 signals at three different frequencies. However, we had no way of knowing if signals observed at a given frequency and time originated from a source whose emission we might also have recorded at a different frequency during our measurement campaign.

Acquisition of signals over uninterrupted $60 \mathrm{~s}$ time intervals allowed us to observe unexpected radar behavior that might have otherwise been missed, as described in Section 3.4.

\subsubsection{Relevant variables and potential sampling biases}

Controlled experimental variables included the local oscillator frequency used for IQ demodulation, the receiver antenna, receiver noise floor (via the VST's reference level), and the triggering and data retention criteria (discussed in the next subsection).

Because the measurements were observational in nature, and collected in an uncontrolled environment, there were several uncontrolled experimental variables, including

- Number, locations, and movements of shipborne SPN-43 radar transmitters

- Operating frequencies of SPN-43 radars

- Presence of non-SPN-43 RF emitters

- Weather conditions, including atmospheric and sea conditions

- Seaborne scatterers, such as watercraft 


\section{- Tropospheric scatter}

- Topographical and structural scatterers, such as nearby hillsides and man-made structures.

Some aspects of the RF and weather conditions can potentially be inferred from our measurements. However, knowledge of the other uncontrolled variables, particularly those concerning the SPN-43 radar and ship movements (i.e., location and orientation), was necessarily limited due to the sensitive nature of naval deployments. Consequently, the set of collected measurements likely suffers from sampling biases that are difficult to quantify without additional information. For example, received power is a function of distance and off-axis angle with the cavity-backed spiral antenna, each of which might cause different distortions to the received pulses. High SNR pulses will result from sources that are close and off axis as well as from emitters that are far away and on axis.

Additionally, there was an unavoidable selection bias in our measurements due to the limited geographical location and experimental timeframe, and as explained below, the triggering and data retention criteria, and the $10 \%$ measurement duty cycle. For these reasons, the sample was not likely representative of the entire population of all SPN-43 radar emissions. Unfortunately, characterization of this selection bias is not possible, since it requires access to a larger, comprehensive sample of SPN-43 emissions, which is not available.

\subsubsection{Trigger and data retention criteria}

A diagram showing our trigger and data retention algorithm is provided in Fig. 2.5. In this section we describe in more detail the trigger criteria and how metadata is generated.

From our VST, we collect in-phase (I) and quadrature (Q) samples at a rate of $225 \mathrm{MSamples} / \mathrm{s}$. We use this sampling rate because it provides an effective $200 \mathrm{MHz}$ bandwith and is also streamable to a fast RAID without dropping samples. In contrast, using a $250 \mathrm{MSamples} / \mathrm{s}$ sampling rate provides a flatter $200 \mathrm{MHz}$ bandwidth, but drops samples while streaming to storage. After a data set is collected to a fast RAID, we have a vector of 16-bit integers that is composed of interleaved $\mathrm{Q}$ and I samples. The structure of this vector can be visualized as: $\left[Q_{1} I_{1} Q_{2} I_{2} Q_{3} I_{3} \cdots Q_{N} I_{N}\right]$, where $N$ represents the number of samples taken. We de-interleave these values and scale them to double precision to obtain the complex vector $\overrightarrow{I Q}$. This complex vector has the form $\overrightarrow{I Q}=$ $\vec{I}+i \vec{Q}$. Specifically, we collect $51 \mathrm{GiB}$ of samples spanning a time period slightly over $60 \mathrm{~s}$. Each complex IQ sample is composed of four bytes before scaling to double precision. To be precise, $\overrightarrow{I Q}$ has a length of 13685760000 samples.

$\overrightarrow{I Q}$ is large enough that it is costly in terms of time and memory to load and analyze. Our wish to examine this data in a time efficient manner dictates that we generate metadata; descriptions 


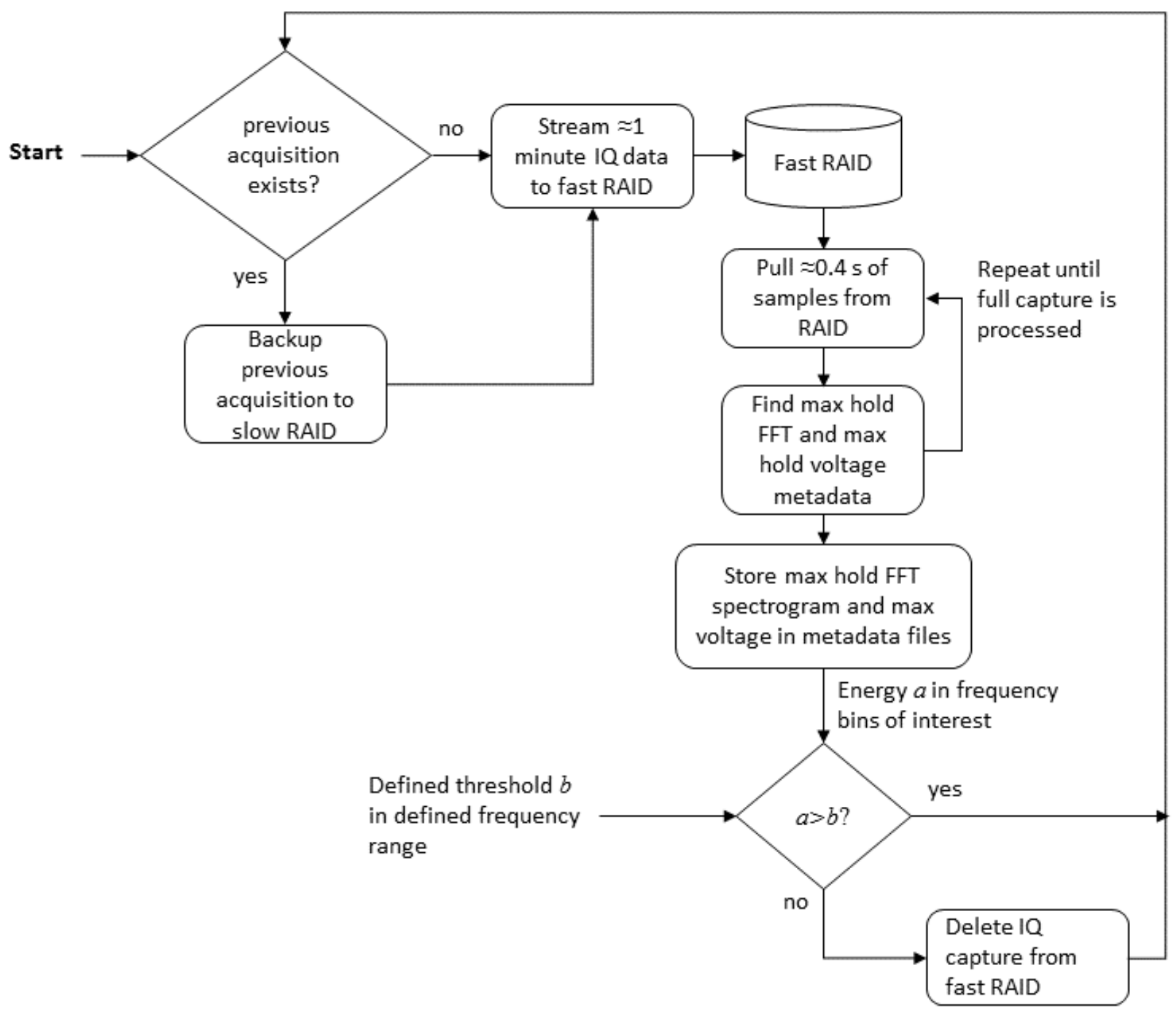

Figure 2.5: Flow chart of acquisition method. After storage of 1 minute of IQ data to the fast RAID, the extraction of metadata requires about 9 minutes of processing, leading to a $10 \%$ measurement duty cycle. 
of this data that are less memory intensive. We specifically use a spectrogram and a max-hold amplitude vector as metadata.

To generate metadata, we load sequential subsets of $\overrightarrow{I Q}$ of length $1024 \times 10^{5}$ samples. Let us represent the $i^{\text {th }}$ subset of $\overrightarrow{I Q}$ as $\overrightarrow{S_{i}}, i \in\{1,2, \ldots, 134\}$. We should note that the last subset, $\overrightarrow{S_{134}}$, is shorter than $1024 \times 10^{5}$ samples due to the total number of samples being a non-integer multiple of $1024 \times 10^{5}$. We also note that $1024 \times 10^{5}$ samples represent about 0.455 seconds of recorded signal. For each $\vec{S}_{i}$ we calculate two pieces of information: a scalar $A_{i}$, the maximum amplitude of $\vec{S}_{i}$, and a vector $\vec{F}_{i}$, the max-hold spectra of $\vec{S}_{i}$. We calculate $\vec{F}_{i}$ using a short-time Fourier transform and retain the maximum amplitude in each frequency bin. With $A_{i}$ and $\vec{F}_{i}$ concatenated over $i$ we obtain a max-hold amplitude vector and a max-hold spectrogram, respectively. The max-hold amplitude information is useful for verifying that the linear range of the VST has not been exceeded. The max-hold spectrogram is useful for visualizing the qualitative behavior of the observed signals, which will be the subject of much of Chapter 3.

We also apply a threshold to the max-hold spectrogram to determine if we wish to retain the raw IQ data or delete it. The threshold compares a subset of the spectrogram with a fixed value. ${ }^{2}$ For example, we typically used a portion of the spectrogram corresponding to $3522 \mathrm{MHz}$ to $3673 \mathrm{MHz}$. If any amplitudes in this portion of the spectrogram exceeded the threshold then we retained the raw data. The reason for deleting data that did not exceed the threshold was to extend the time we could collect data to weeks or months before reaching storage capacity.

The time required to load the data back into the CPU and calculate the metadata is about nine minutes, leading to an acquisition duty cycle of $10 \%$.

\subsection{Measurement calibration}

We characterized the individual system components to verify the system gain and gain flatness, and to ensure that the system would be stable with respect to temperature variations that might be encountered with field deployment. We also characterized the system noise and linearity and the gain of our two antennas. Our characterization procedures are described in Sections 2.3.1 through 2.3.7 and the results are summarized and discussed in Section 2.3.8. Detailed response measurements are plotted at the end of the chapter.

\footnotetext{
${ }^{2}$ We used a threshold that corresponds to a power of approximately $-76.7 \mathrm{dBm}$ in a frequency bin, referenced to the output of the antenna.
} 


\subsubsection{RF section magnitude response}

We estimated the gain and associated uncertainty for the RF path between the output of the antenna and the input of the VST, which included the following three components: a short cable from the output of the antenna to the input of the pre-selector; the $3.5 \mathrm{GHz}$ path of the pre-selector; and a long cable from the output of the pre-selector to the input of the VST (see Fig. 2.4).

The measurements of the pre-selector gain were performed using a metrology-grade vector network analyzer (VNA) corrected with a Type-N open-short-load-thru (OSLT) calibration using the Microwave Uncertainty Framework [11], which allows the physical error mechanisms in the calibration standards to be propagated to the measurements of the components under test, and statistical correlations to be determined between both the scattering parameters at a single frequency and uncertainties at different frequencies. Additionally, the pre-selector was measured in an environmental chamber to take temperature dependence into account. The short and long cables, as well as the pre-selector with the replaced $3.5 \mathrm{GHz}$ bandpass filter, were measured on location using a portable VNA with built-in Type-N OSLT calibration.

The reported measurement values and uncertainties (represented as $95 \%$ confidence intervals) were determined for the frequency range between $3570 \mathrm{MHz}$ to $3630 \mathrm{MHz}$. Fig. 2.6 plots the magnitudes of the transmission coefficients $\left(S_{21}\right)$ for the pre-selector's $3.5 \mathrm{GHz}$ path as functions of frequency and temperature along with uncertainties. The pre-selector was measured at four distinct temperatures: $11^{\circ} \mathrm{C}, 20^{\circ} \mathrm{C}, 29^{\circ} \mathrm{C}$, and $38^{\circ} \mathrm{C}$. For the $3570 \mathrm{MHz}$ to $3630 \mathrm{MHz}$ frequency range, the average gain is approximately $29.15 \mathrm{~dB}$ with an uncertainty of $\pm 0.7 \mathrm{~dB}$, which accounts for repeatability, frequency dependence, and temperature dependence. Figs. 2.7 and 2.8 plot the preselector's out-of-band rejection at four different temperatures, and show that out-of-band rejection is nearly constant with temperature.

After replacing the original $3.5 \mathrm{GHz}$ bandpass filter in the pre-selector with a narrower bandpass filter (see Section 2.1.2), we re-measured the gain of the pre-selector with the portable VNA at Fort Story, and calculated an average gain of $29.03 \mathrm{~dB}$ between $3570 \mathrm{MHz}$ and $3630 \mathrm{MHz}$. Since this value was close to that of the original filter, we chose to use an average gain of $29.1 \mathrm{~dB}$. Figs. 2.9 and 2.10 plot the magnitudes of $S_{21}$ for both the old and new filters.

Fig. 2.11 plots the magnitudes of $S_{21}$ for the long cable measured with the portable VNA. The average loss was $5.85 \mathrm{~dB}$. Since we were not able to perform temperature-dependent measurements on this cable, we chose to apply the cable manufacturer's specified uncertainty of $\pm 3.5 \%$ (or $\pm 0.3 \mathrm{~dB}$ ) for the temperature range between $11^{\circ} \mathrm{C}$ and $38^{\circ} \mathrm{C}$. Fig. 2.12 plots the magnitudes of $S_{21}$ for the short cable, also measured with the portable VNA. Once again, we chose to apply an uncertainty of $\pm 0.3 \mathrm{~dB}$.

Table 2.1 summarizes our average measurements of gain for the three components, along with 


\begin{tabular}{|c|c|}
\hline Component & Gain \pm Uncertainty (dB) \\
\hline Front-End 3.5 GHz Path & $+29.1 \pm 0.7$ \\
Short Cable & $-1.03 \pm 0.3$ \\
Long Cable & $-5.85 \pm 0.3$ \\
\hline Net Gain & $+\mathbf{2 2 . 2} \pm \mathbf{0 . 9}$ \\
\hline
\end{tabular}

Table 2.1: RF path gain from the output of the antenna to the input of the VST averaged from 3570 to $3630 \mathrm{MHz}$ and averaged over all temperatures.

their respective uncertainties. The table also includes the overall net gain and uncertainty. The overall uncertainty was determined by a root-sum-of-squares calculation, rounded up to the nearest tenth of a decibel.

\subsubsection{RF section group delay}

We calculated the effect due to group delay distortion of the pre-selector by Fourier transforming typical pulses measured at Virginia Beach, multiplying by $S_{21}$ of the pre-selector and inverse Fourier transforming. The pulses were observed to change in duration by less than the sampling resolution of our instrumentation, i.e., 4.4 ns.

\subsubsection{VST response magnitude}

A typical measurement of the VST response magnitude at room temperature is shown in Fig. 2.13. The manufacturer specifies the typical flatness of the VST magnitude response as $\pm 0.5 \mathrm{~dB}$ and the absolute accuracy $\pm 0.8 \mathrm{~dB}$ (95\% confidence interval) over a temperature range of $\pm 5^{\circ} \mathrm{C}$ after running the internal calibration routine. Due to a programming error, we did not run the internal calibration routine when the system was deployed. It was therefore necessary to characterize the VST response as a function of temperature without running the internal calibration routine. For this measurement, we used a VNA with an additional power calibration to deliver a $+5 \mathrm{dBm}$ sine-wave to the VST input, which was set to the $+5 \mathrm{dBm}$ reference level. After settling at the temperatures (in order) $25^{\circ} \mathrm{C}, 30^{\circ} \mathrm{C}$, and $20^{\circ} \mathrm{C}$, the VST measured the sine wave without running the internal calibration routine. This allowed us to test the absolute accuracy of the VST when the temperature had changed by $5^{\circ} \mathrm{C}$ or $10^{\circ} \mathrm{C}$. Also, the response of the VST is not flat with frequency and ramps up from the local oscillator (LO) frequency $f_{L O}$ at a rate of about $0.01 \mathrm{~dB} / \mathrm{MHz}$. The VST was generally found to attenuate the signal by $1.7 \mathrm{~dB} \pm 0.6 \mathrm{~dB}$, where the $0.6 \mathrm{~dB}$ standard uncertainty [12] includes effects of gain drift over a $10^{\circ} \mathrm{C}$ temperature range $(0.23 \mathrm{~dB})$, repeatability of the measurements $(0.23 \mathrm{~dB})$, and the flatness of the response with frequency $(0.5 \mathrm{~dB})$. 
As mentioned above, the sample rate of the ADCs in the VST is $250 \mathrm{MSamples} / \mathrm{s}$. We believe that this rate is fixed and lower sample rates are achieved by interpolation and sub-sampling. Fig. 2.13 shows the out-of-band rejection of the VST anti-aliasing filter. ${ }^{3}$ For input signal frequencies less than $f_{N 1}=f_{L O}-f_{S} / 2=(3577-112.5) \mathrm{MHz}=3464.5 \mathrm{MHz}$, spurious tones occur near $f_{N 1}$ and near $f_{L O}+112.5=3689.5 \mathrm{MHz}$. For input frequencies between $f_{N 2}$ and $f_{N 1}$ the later of these tones appear between $3675 \mathrm{MHz}$ and $3689.5 \mathrm{MHz}$ and these are the tones used to calculate the out-of-band rejection. Due to the interpolation and sub-sampling method used in the VST, the exact frequency of all these spurs is a complicated function of both $f_{N 1}$ and $f_{N 2}=f_{L O}-f_{S, \max } / 2-\left(f_{S, \max }-f_{S}\right) / 2=(3577-125-12.5) \mathrm{MHz}=3439.5 \mathrm{MHz}$ and will not be discussed further here.

The net out-of-band rejection of the original pre-selector's filter plus the VST's anti-aliasing filter is shown in Fig. 2.14, with approximately $12 \mathrm{~dB}$ rejection at $f_{N 1}$ and between $47 \mathrm{~dB}$ and $67 \mathrm{~dB}$ rejection at $f_{N 2}$.

The net out-of-band rejection of the new pre-selector's filter plus the VST's anti-aliasing filter is shown in Fig. 2.15, with approximately $45 \mathrm{~dB}$ rejection at $f_{N 1}$ and between $121 \mathrm{~dB}$ and $136 \mathrm{~dB}$ rejection at $f_{N 2}$. This places tones at $3440 \mathrm{MHz}$, that are aliased into the measured band, well below the VST noise floor (see Sections 2.3.6 and 2.3.7).

\subsubsection{VST local oscillator and sampling frequencies}

The pulse parameters of Chapter 4 depend critically on the frequency accuracy of the VST. While the manufacturer specifies the internal frequency reference accuracy of the VST as \pm Aging \pm Temperature stability $= \pm 2 \times 10^{-6}$ per year, maximum, it is not clear how the LO frequency and sampling rate are affected by the frequency reference accuracy. Therefore, it is important that the LO frequency and sampling rate accuracy be verified. To do this we used a VNA whose $10 \mathrm{MHz}$ reference was tied to one of the NIST Hydrogen masers as a signal source [13]. The VST LO frequency was set to $3565 \mathrm{MHz}^{4}$ and we measured records of $1 \mathrm{~ms}$ or longer with input signal frequencies greater than and less than $3565 \mathrm{MHz}$. For measured data at each frequency, we performed an FFT on the time record and made note of the calculated peak signal frequency. We also used an adaptive algorithm to estimate the signal frequency with sub FFT-bin resolution. We expect that measured frequency errors would have the same sign if the LO frequency were in error, while the measured frequency grid would be stretched or contracted if the sampling rate were in error. A combination of these effects would result in an error verses offset from the LO frequency having

\footnotetext{
${ }^{3}$ We focus here on frequencies below the observed frequency range because we did not expect or observe strong signals at frequencies higher than $3650 \mathrm{MHz}$.

${ }^{4}$ Errors in LO frequency and sampling rate were characterized for measurements at Point Loma [1], but the results should be independent of the specific LO frequency.
} 
some mean offset and trend. The measurements showed a general offset but no significant trend, indicating that the $\mathrm{LO}$ frequency error dominates. The mean LO frequency was found to be in error by $+3.06 \pm 0.17 \mathrm{kHz}$ (from the mean and standard deviation of eight measurements symmetrically distributed around the LO frequency), or slightly less than the manufacturer's specified 1 part in $10^{6}$ accuracy.

\subsubsection{Antenna characterization}

We attempted a spot characterization of each of our antennas: a $45^{\circ}$ (linear) slant polarization omni-directional antenna and a cavity-backed spiral antenna. However, it should be noted that we cannot compensate for the antenna gain or antenna pattern in our measurements because we do not know the position of the emitters or the path that the signals take between the emitter and our measurement system. The analysis of subsequent chapters is referenced to a calibration plane at the coaxial output of the antenna. The antenna characterization performed here is used to fully specify the measurement system.

The antennas were characterized in a $10.4 \mathrm{~m} \times 5.6 \mathrm{~m} \times 4.1 \mathrm{~m}$ anechoic chamber (from cone tip to cone tip) by use of a conical spiral reference antenna positioned $3 \mathrm{~m}$ from antenna under test. The gain of the reference antenna was measured by the manufacturer and we estimate that it was characterized with uncertainty $U_{\text {ref }}=1 \mathrm{~dB}$.

The mean gain of the omni-directional antenna, variation in gain with azimuth, gain standard uncertainty, and gain expanded uncertainty at the $68 \%$ confidence level are given in Table 2.2.

To measure the horizontal gain and azimuthal dependence of the omni-directional antenna, it and the reference antenna were mounted $\approx 2.3 \mathrm{~m}$ from the floor of the anechoic chamber. Measurements were taken at the cardinal directions of $0^{\circ}, 90^{\circ}, 180^{\circ}, 270^{\circ}$, and $360^{\circ}$, where measurements at $0^{\circ}$ and $360^{\circ}$ can be used to characterize the positioning repeatability of the measurements.

We estimate the positioning repeatability uncertainty $U_{r}$ with one degree of freedom as

$$
U_{r}=\sqrt{\left(\overline{G\left(0^{\circ}\right)}-G\left(0^{\circ}\right)\right)^{2}+\left(\overline{G\left(0^{\circ}\right)}-G\left(360^{\circ}\right)\right)^{2}}
$$

where,

$$
\overline{G\left(0^{\circ}\right)}=\frac{1}{2}\left(G\left(0^{\circ}\right)+G\left(360^{\circ}\right)\right) .
$$

We use the mean gain $\bar{G}$ and the standard deviation $S_{G}$ of the gain $G\left(\phi_{i}\right)$ over all azimuthal angles $\phi=\left(0^{\circ}, 90^{\circ}, 180^{\circ}, 270^{\circ}\right)$, where $G\left(0^{\circ}\right)=\overline{G\left(0^{\circ}\right)}$, to quantify the gain and gain uniformity of the antenna. $S_{G}$ is found with three degrees of freedom. 


\begin{tabular}{|cccccc|}
\hline Frequency $(\mathbf{M H z})$ & $\bar{G}(\mathrm{dBi})$ & $U_{r}(\mathrm{dBi})$ & $S_{G}(\mathrm{dBi})$ & $U_{G}(\mathrm{dBi})$ & $U_{G} k_{0.68}(\mathrm{dBi})$ \\
\hline 3570 & 0.7 & 0.4 & 0.9 & 1.4 & 1.6 \\
3600 & 0.9 & 0.8 & 0.7 & 1.4 & 1.6 \\
3630 & 1.6 & 0.5 & 0.4 & 1.2 & 1.4 \\
\hline
\end{tabular}

Table 2.2: Estimated gain of omni-directional antenna in the horizontal plane, averaged over cardinal directions, with uncertainty at three frequencies. Variables are defined in the text.

The standard uncertainty of the gain, which quantifies the uncertainty in the gain experienced when measuring any particular signal, is found by adding the uncertainties in quadrature:

$$
U_{G}=\sqrt{U_{r}^{2}+S_{G}^{2}+U_{\text {ref }}^{2}}
$$

Since we have a limited number of measurements, we next find the expanded uncertainty for a $p=0.68$ confidence interval. First we find the effective number of degrees of freedom $\nu_{\text {eff }}$ by use of the Welch-Satterthwaite equation [12]

$$
\nu_{\mathrm{eff}}=\frac{U_{G}^{4}}{S_{G}^{4} / 3+U_{r}^{4}},
$$

and then find $k_{p}=t_{p}\left(\nu_{\text {eff }}\right)$, where $t_{p}\left(\nu_{\text {eff }}\right)$ is the $t$-distribution, for degrees of freedom $\nu_{\text {eff }}$, that defines an interval $-t_{p}\left(\nu_{\text {eff }}\right)$ to $+t_{p}\left(\nu_{\text {eff }}\right)$ that encompasses the fraction $p$ of the distribution. Here we have assumed that the degrees of freedom of the reference antenna gain is a large number. Note that $\nu_{\text {eff }}$ is a function of the relative contributions of the different uncertainty components and is therefore not fixed with frequency, varying here between 10 and 23.

Our observed gain agrees well with the manufacturer's characterization of the antenna. However, the manufacturer indicates maximum gain roughly at $60^{\circ}$ above the horizon and roughly $5 \mathrm{~dB}$ to $7 \mathrm{~dB}$ higher than at the horizon.

The omni-directional antenna is specified as a "slant polarization" antenna by the manufacturer, and has maximum input coupling when the incident signal is linearly polarized at $45^{\circ}$ to horizontal. Horizontally polarized and circularly polarized signals will experience $3 \mathrm{~dB}$ loss.

The CBS antenna is right-hand circular polarized and we expect $3 \mathrm{~dB}$ coupling loss into the antenna when the SPN-43 is linearly polarized and tens of $\mathrm{dB}$ coupling loss when the SPN-43 is left-hand circularly polarized.

To measure the CBS antenna gain, the antenna was mounted on a polyvinyl chloride (PVC) pipe, which in turn was mounted on a kinematic mount capable of rotation in $15^{\circ}$ increments. The CBS antenna pattern, measured in $15^{\circ}$ increments, is shown in Fig. 2.16 and boresight gain is 


\begin{tabular}{|ccc|}
\hline Frequency (MHz) & Boresight gain (dBi) & Uncertainty (dBi) \\
\hline 3570 & 6.3 & 1 \\
3600 & 6.9 & 1 \\
3630 & 7.2 & 1 \\
\hline
\end{tabular}

Table 2.3: Boresight gain of CBS antenna at three frequencies with estimated uncertainty. Note that uncertainties at off axis angles may be greater

\begin{tabular}{|cc|}
\hline Reference level $(\mathbf{d B m})$ & Noise equivalent power $\mathbf{( d B m} / \mathbf{H z})$ \\
\hline 5 & -158.1 \\
0 & -161.5 \\
-10 & -165.7 \\
-20 & -166.9 \\
-30 & -167.3 \\
\hline
\end{tabular}

Table 2.4: System noise equivalent power, referenced to the antenna output for various VST reference level settings. The measurements analyzed, qualitatively or quantitatively, in this document were conducted at a reference level of $5 \mathrm{dBm}$ unless otherwise noted.

given in Table 2.3. Here the dominant contributions to the uncertainty in the boresight gain are the reference antenna gain and the orientation error.

\subsubsection{System noise floor}

The system noise floor was measured in the field by connecting a $50 \Omega$ coaxial termination at the end of the short cable where the antenna would normally be located, acquiring a $60 \mathrm{~s}$ temporal record, and then calculating the spectrogram metadata. The system noise floor, referenced to the short cable input, was then calculated based on spectrograms, the RF section magnitude response, and the VST response magnitude, (see Sections 3.2, 2.3.1, and 2.3.3, respectively, for more information) and verified with the full IQ waveform files. The measured noise equivalent power, referenced to the antenna output, is given in Table 2.4.

\subsubsection{System nonlinearity}

System linearity was a major consideration in both the design of the system and analysis of the measurement data. The pre-selector uses an LNA whose $-1 \mathrm{~dB}$ compression point is $27.9 \mathrm{dBm}$. The VST was typically run with the reference level at $5 \mathrm{dBm}$ or lower, meaning that the VST would saturate at much lower power than the LNA. 


\begin{tabular}{|c|c|}
\hline Component & Gain \pm Uncertainty (dB) \\
\hline RF section & $+22.2 \pm 0.9$ \\
VST & $-1.7 \pm 0.6$ \\
\hline Omni-directional antenna & (0.7 to 1.6$) \pm(1.6$ to 1.5$)$ \\
CBS antenna (on boresight) & $(6.3$ to 7.2$) \pm 1$ \\
\hline
\end{tabular}

Table 2.5: Summary of component gains. See discussion of uncertainties due to angle of arrival in Section 2.3.8.

The VST uses ADCs with 14-bit resolution. The spur-free dynamic range (SFDR) of the VST was calculated, when characterizing its gain, and was shown to be typically between $65 \mathrm{~dB}$ and $70 \mathrm{~dB}$ with a sinusoidal input signal and power equal to the reference level. At a reference level of $+5 \mathrm{dBm}$ the VST did not saturate until $\mathrm{CW}$ input power exceeded $+10 \mathrm{dBm}$.

The max-hold metadata $A_{i}$ (in the time domain) and $\vec{F}_{i}$ (in the frequency domain) were used for checking for VST saturation post facto. Peak voltages $A_{i}$ were confirmed to be lower than the reference level plus margin, e.g., see Fig. 4.1. Files containing spurs that might have been caused by receiver nonlinearity were rejected from analysis, as described in [1, Appendix A].

\subsubsection{Summary and discussion of system gain and uncertainty}

The component gain measurements described in Sections 2.3.1 through 2.3.7 are summarized in Table 2.5. While we believe that we can characterize the RF section and VST gain, we cannot say the same with the antennas because of the unknown paths of propagation from the source to the measurement antenna and the angle of arrival in both azimuth and elevation. The paths involved include direct line-of-sight propagation, as well as reflections from nearby objects and atmospheric scattering. For the omni-directional antenna, elevation angle could cause as much as $5 \mathrm{~dB}$ to $7 \mathrm{~dB}$ variation, while azimuthal variation could cause roughly $2 \mathrm{~dB}$ variation (see Table 2.2 ). For the CBS antenna, given the approximately $180^{\circ}$ field of view at the measurement site, variation in azimuthal direction of arrival could cause as much as $19 \mathrm{~dB}$ variation.

In conclusion, antenna pattern effects potentially dominate our measurement uncertainties and could severely distort incoming signals, particularly in the case of multiple signal paths with significantly different angles of incidence. However, if we assume that the antennas used in an ESC system are similar to the ones used in our measurements, signals measured by ESCs would be similarly distorted. Therefore, we believe that our measured signals are still representative of those that ESCs would need to detect. 


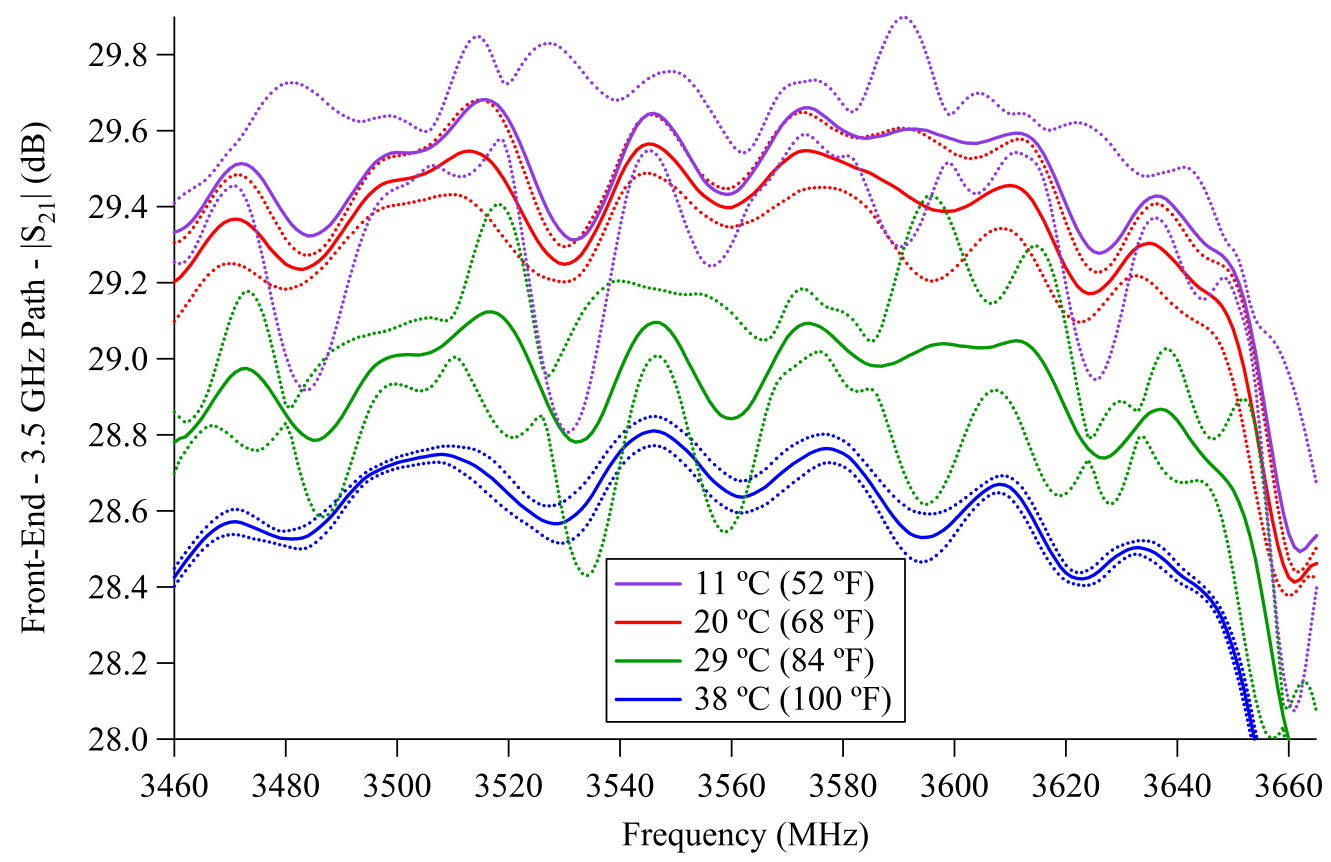

Figure 2.6: Transmission measurements of the pre-selector's original $3.5 \mathrm{GHz}$ path as functions of frequency and temperature along with uncertainties.

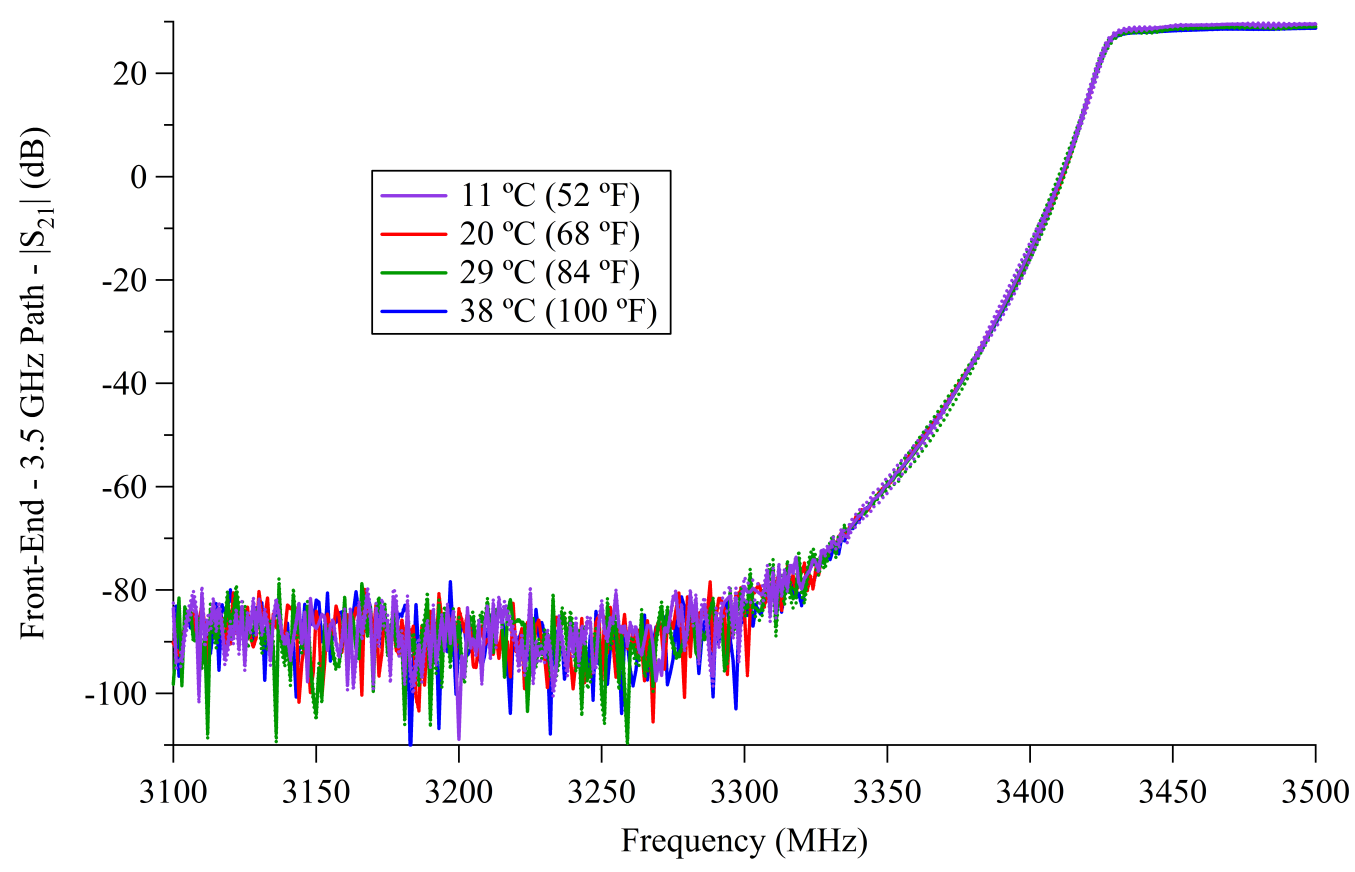

Figure 2.7: Transmission measurements of the pre-selector's original $3.5 \mathrm{GHz}$ path lowfrequency rejection as functions of frequency and temperature. 


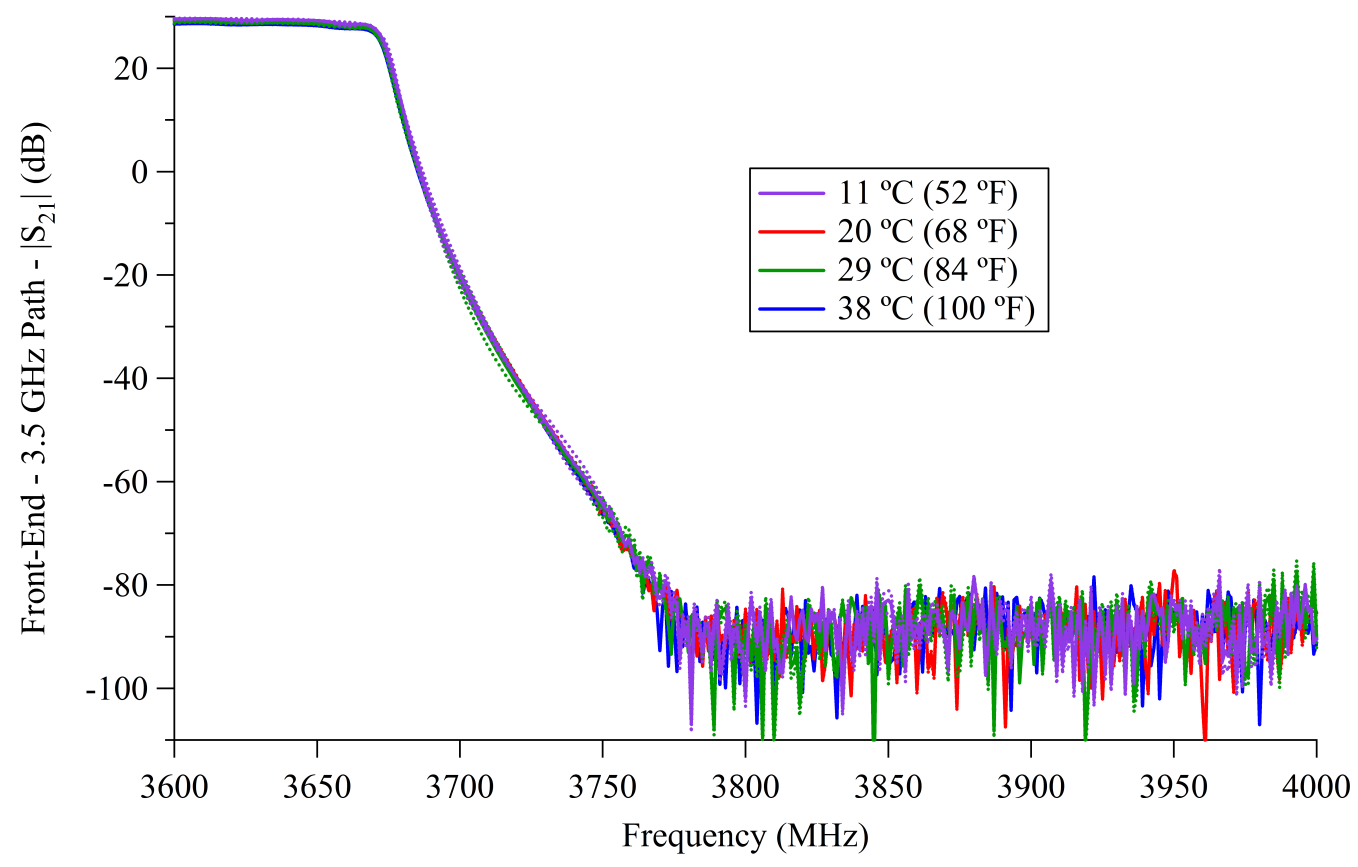

Figure 2.8: Transmission measurements of the pre-selector's original $3.5 \mathrm{GHz}$ path highfrequency rejection as functions of frequency and temperature.

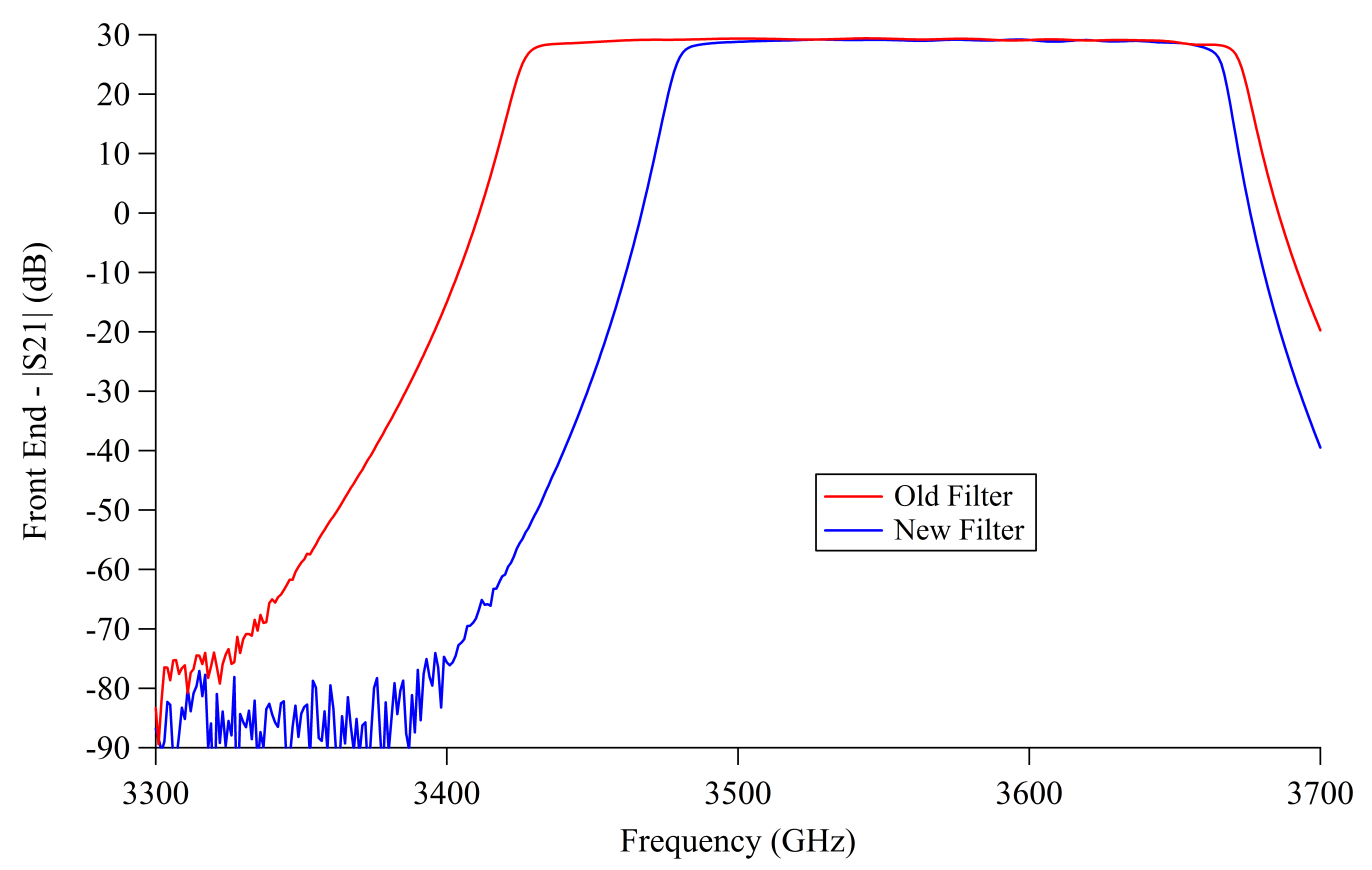

Figure 2.9: Transmission measurements of the old and new pre-selector's $3.5 \mathrm{GHz}$ path as a function of frequency. 


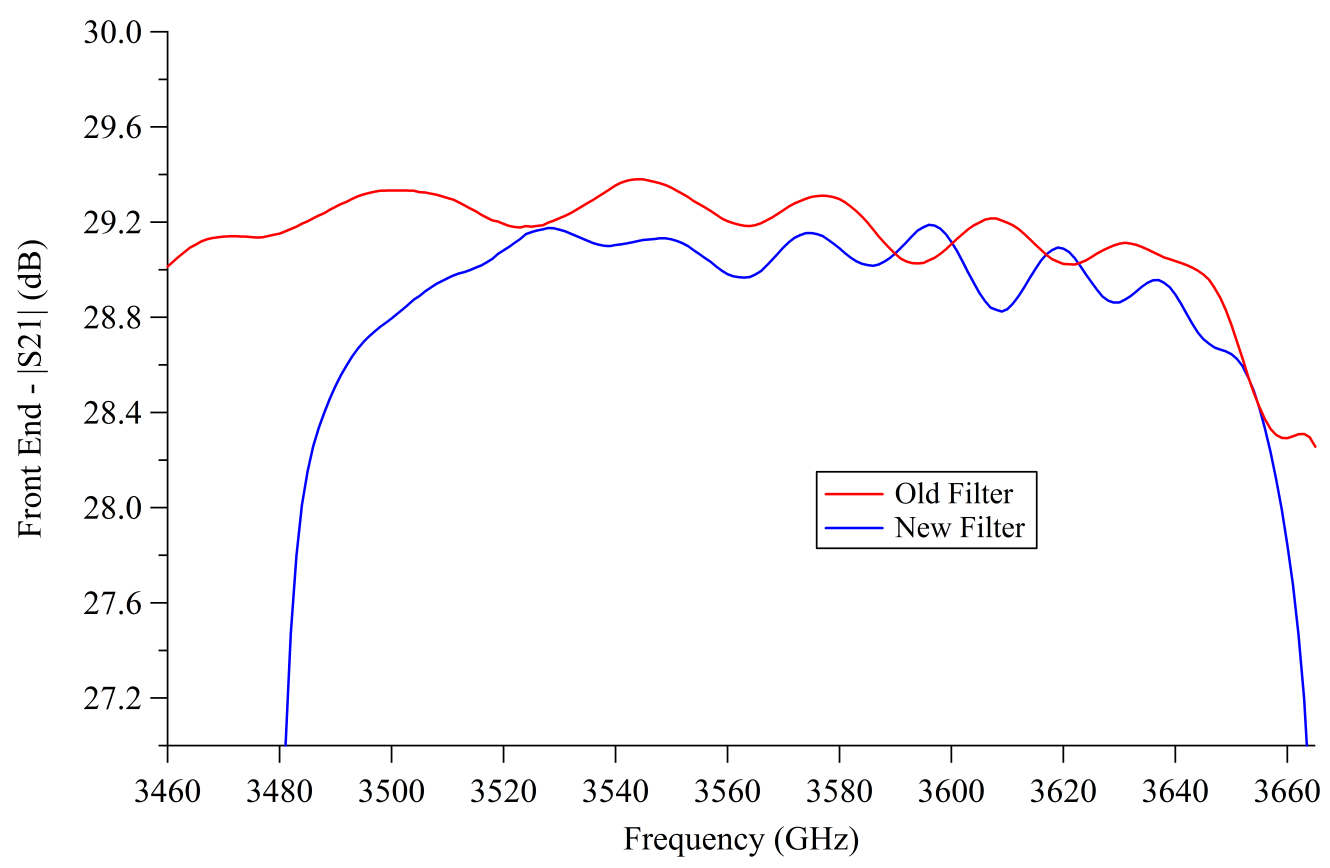

Figure 2.10: Transmission measurements of the old and new pre-selector's $3.5 \mathrm{GHz}$ path as a function of frequency (zoomed in).

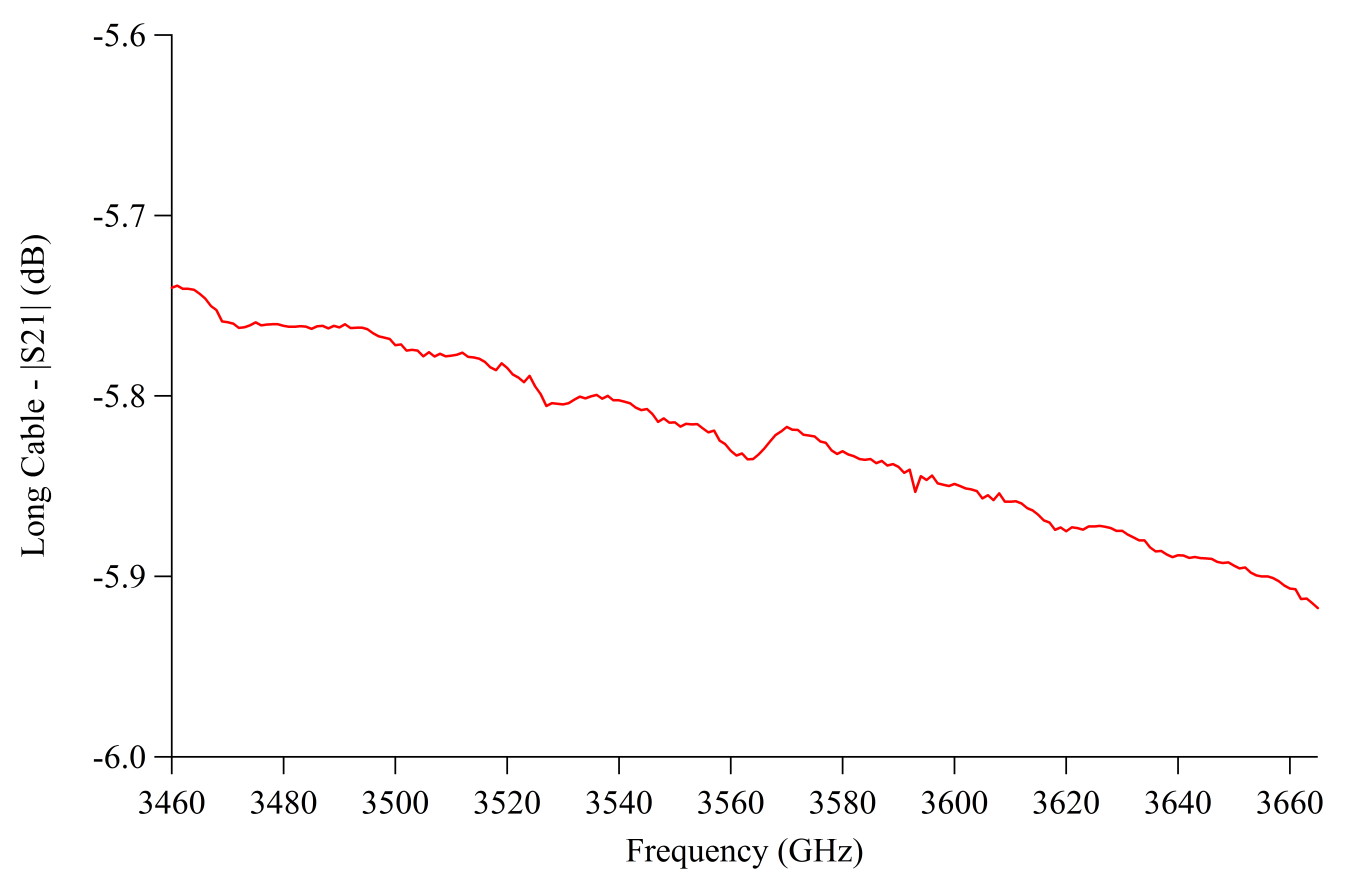

Figure 2.11: Transmission measurements of the long cable as a function of frequency. 


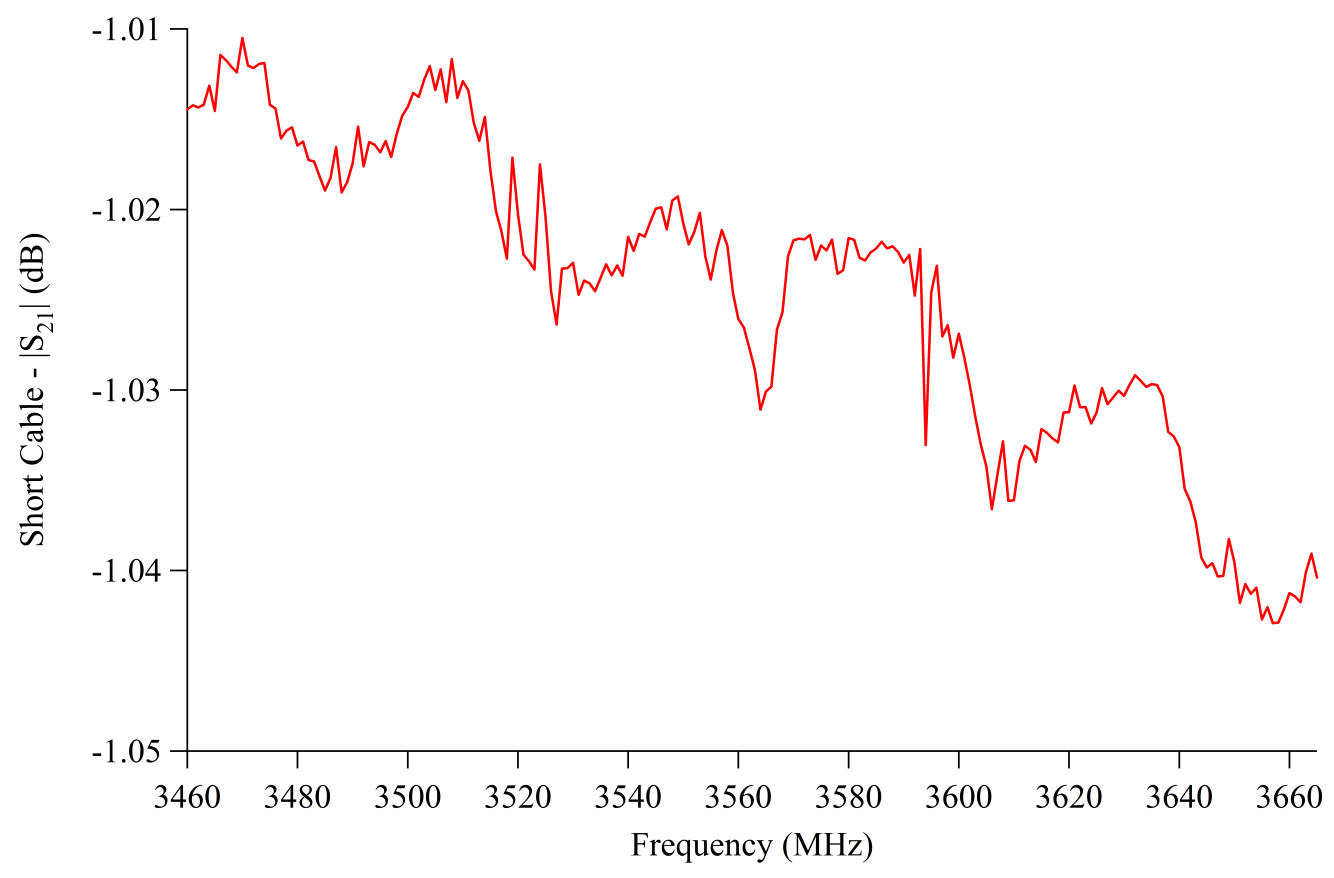

Figure 2.12: Transmission measurements of the short cable as a function of frequency. 


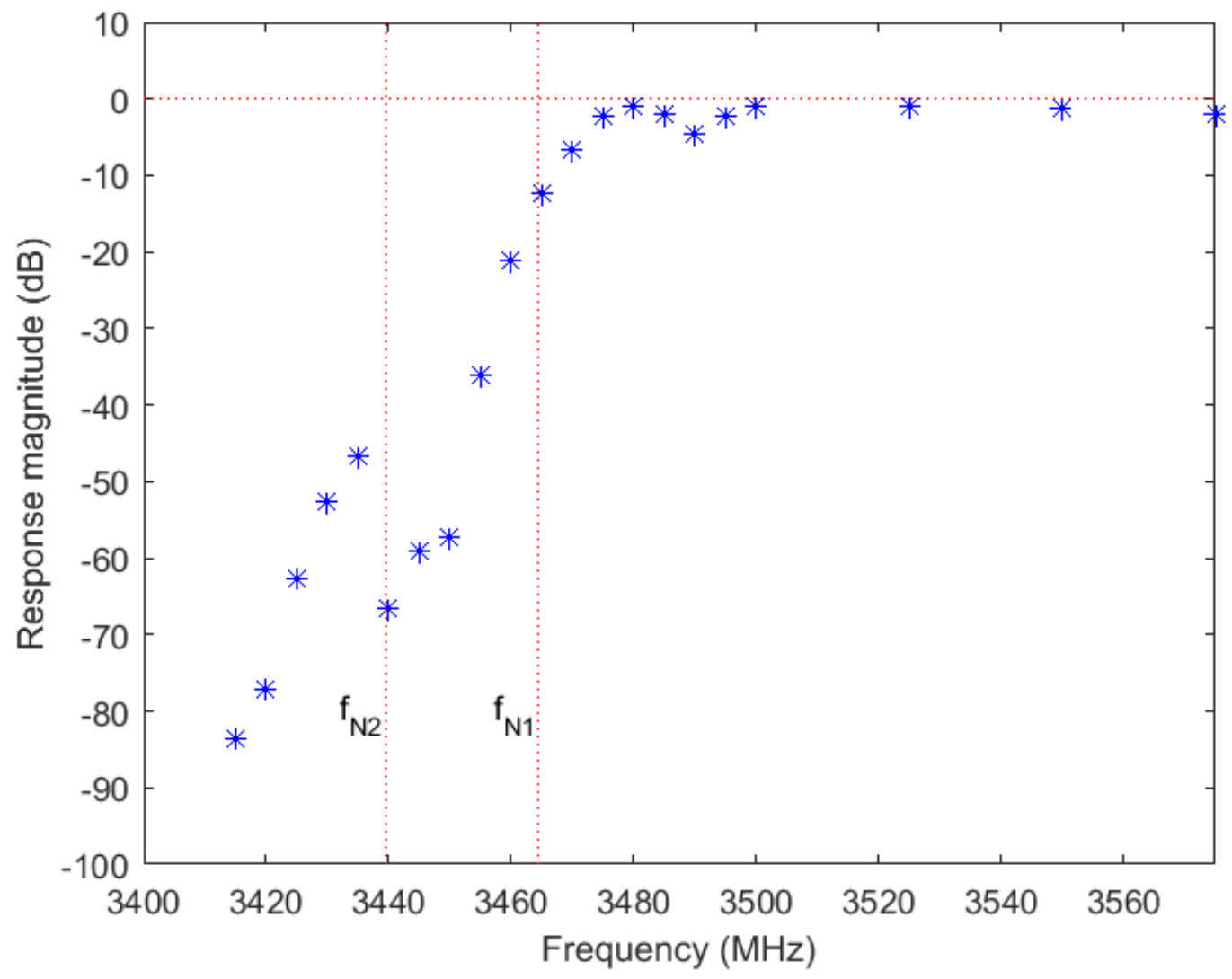

Figure 2.13: Normalized response of the VST measured at room temperature and at select input frequencies below the LO frequency $f_{L O}=3577 \mathrm{MHz}$. The VST reference level and the input power were $+5 \mathrm{dBm}$. Frequencies $f_{N 2}$ and $f_{N 1}$ refer to the Nyquist frequency due to the VST $250 \mathrm{MSample/s} \mathrm{sample} \mathrm{rate} \mathrm{and} \mathrm{the} \mathrm{re-sampling} \mathrm{rate} \mathrm{of} 225 \mathrm{MSample} / \mathrm{s}$, respectively. For input frequencies below $f_{N 1}$ the response is measured at the frequency of the spur appearing above $f_{L O}$. 


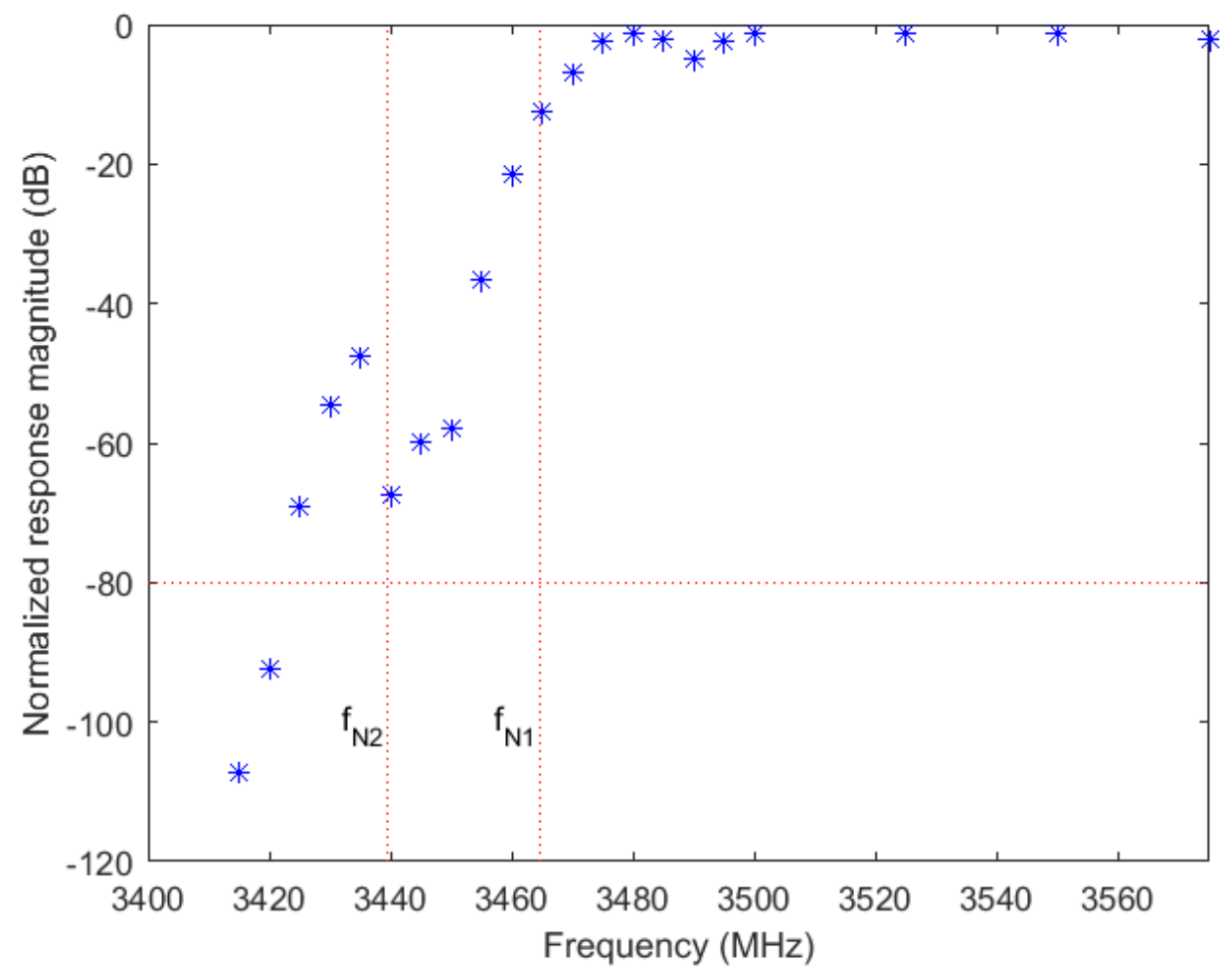

Figure 2.14: Normalized out-of-band gain of pre-selector plus VST measured at room temperature and at select input frequencies below the LO frequency $f_{L O}=3577 \mathrm{MHz}$. Rejection is calculated by summing the room temperature (interpolated) curves in Figs. 2.7 and 2.13, then normalizing to the maximum in-band transmission gain. Horizontal line at $-80 \mathrm{~dB}$ denotes noise floor of VST. 


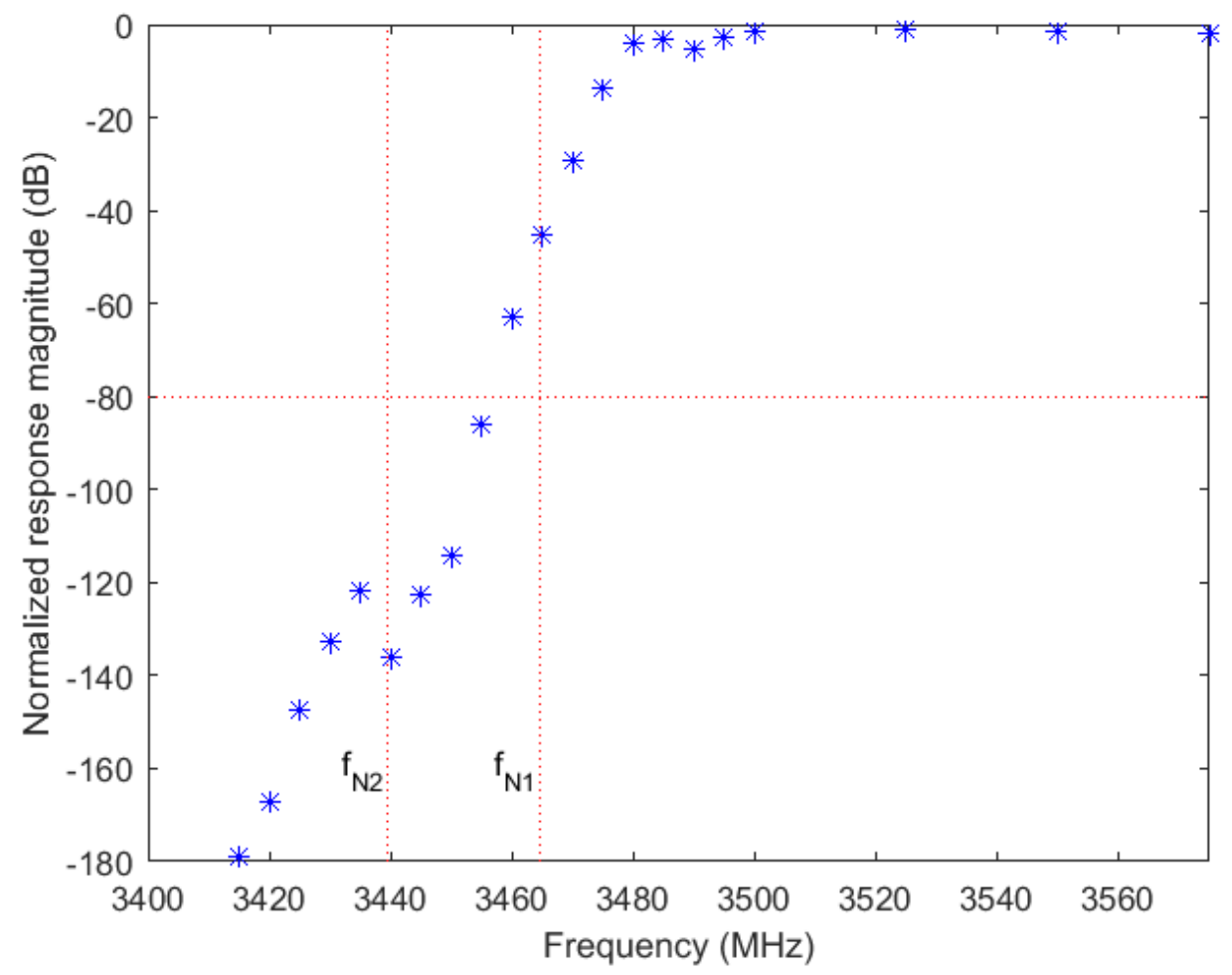

Figure 2.15: Normalized out-of-band gain of pre-selector plus VST measured at room temperature and at select input frequencies below the LO frequency $f_{L O}=3577 \mathrm{MHz}$. Rejection is calculated by summing the room temperature (interpolated) curves in Figs. 2.7 and 2.13, then normalizing to the maximum in-band transmission gain. Horizontal line at $-80 \mathrm{~dB}$ denotes noise floor of VST. 


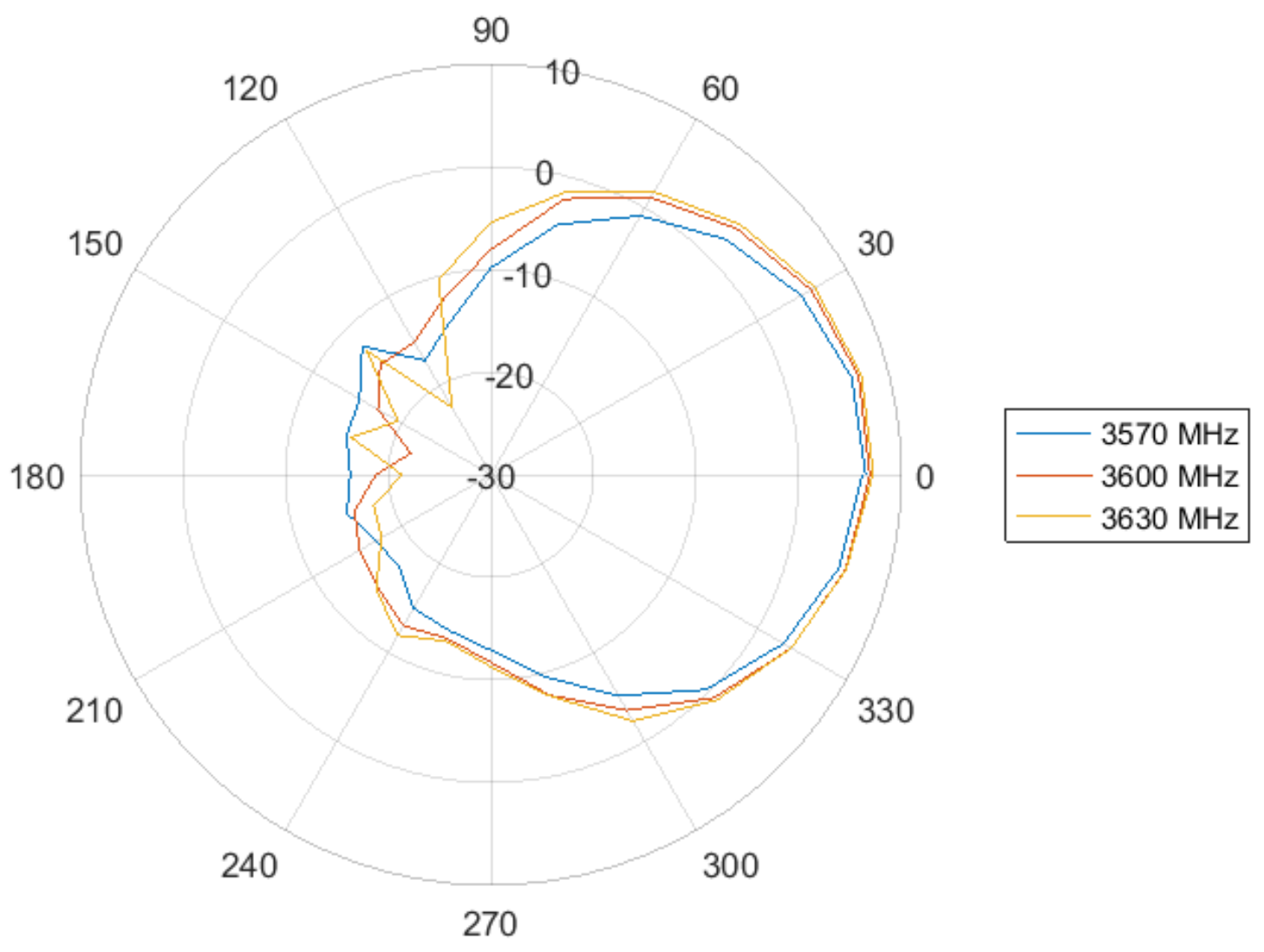

Figure 2.16: CBS antenna gain (dBi) vs. azimuth angle (degrees) at three frequencies. Boresight gain is given in Table 2.3 . The $-3 \mathrm{~dB}$ beam width is roughly $60^{\circ}$ at all frequencies and the ratio of front to back gain is at least $17 \mathrm{~dB}$. 


\title{
Chapter 3
}

\section{Qualitative Results}

\begin{abstract}
Although the library of high-quality IQ waveform data is the key outcome of this work, the spectrogram metadata used for triggering the data acquisition and for diagnostics is also useful for analyzing the qualitative behavior of the signals. Observation of specific events can serve as counter examples to conjectures on how the DoD uses the spectrum. Furthermore, observation of certain unexpected behaviors at Point Loma [1] and at Fort Story gives confidence that these behaviors are not a one-time event. In this chapter we start with an overview of typical SPN-43 waveforms and spectrograms during what could be called normal operation. We then show a few examples of SPN-43 signals that we call anomalous, disproving a few of our initial assumptions. We conclude the chapter with a discussion of observed spectrograms of signals that could interfere with CBRS or ESC operation.
\end{abstract}

\subsection{Overview of SPN-43 waveforms}

Previous to [1] and the current work, public information regarding SPN-43 was limited to the standard SPN-43 radar specifications from 1973 [5], which are summarized in [6]. More current specifications are available in [2] and are summarized in Table 1.1. A limited number of measurements were reported in [6] and [8]. In Chapter 4 we give detailed statistics of our measured antenna rotation period, pulse repetition interval, pulse duration, and frequency offset.

The magnitude of a typical IQ waveform from the Fort Story measurement campaign is shown in Fig. 3.1, where the radar dish rotation is clearly shown as a high peak-power burst of pulses roughly every $4 \mathrm{~s}$. Note that the peak amplitude varies with time and that significant structure is observed between the rotations. The structure may be due to back-lobes of the radar antenna or reflections from neighboring objects, such as the ship's superstructure, other ships, or various 


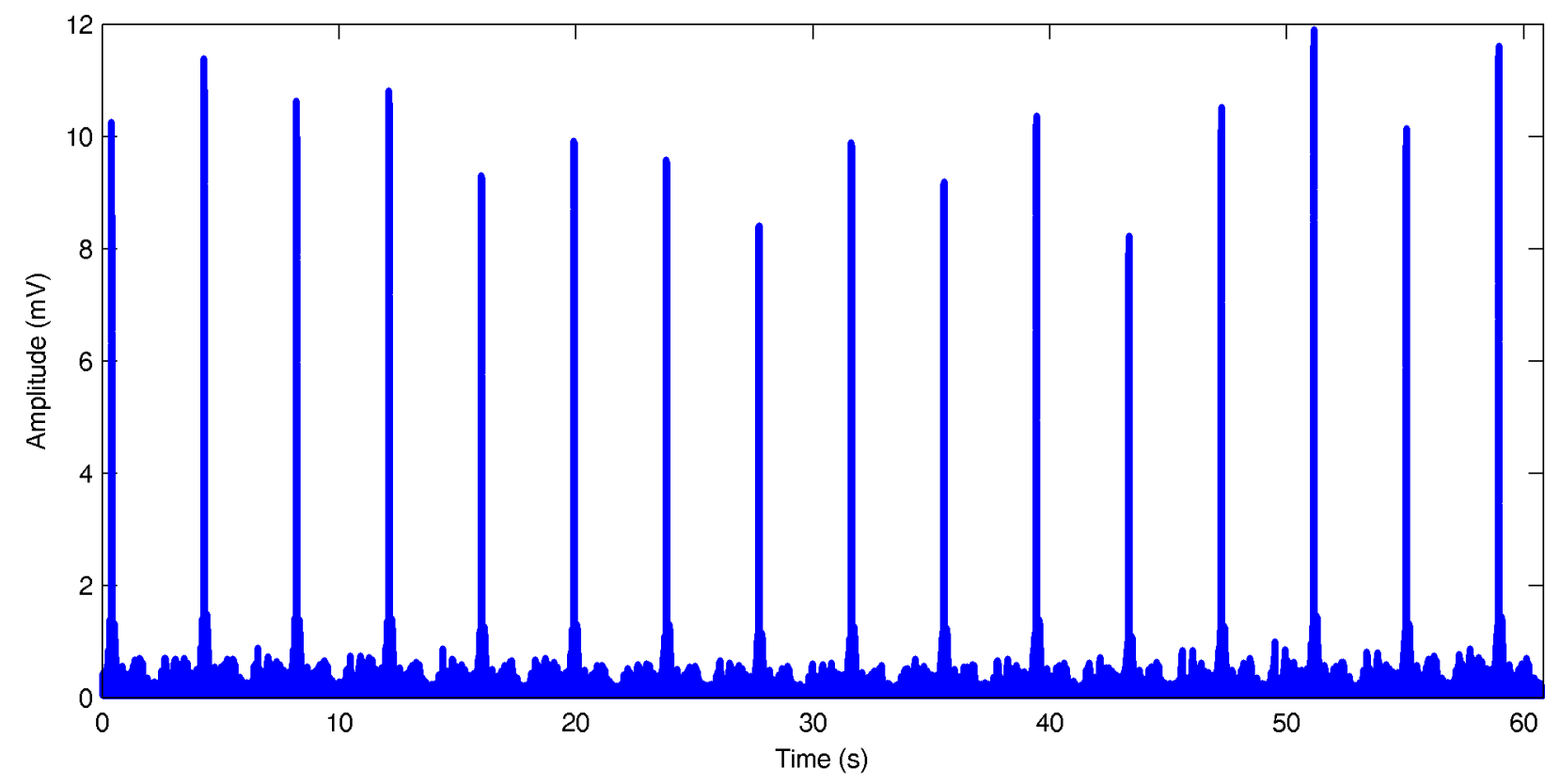

Figure 3.1: Magnitude of an IQ capture of a typical $3570 \mathrm{MHz}$ SPN-43 radar. The full capture epoch of just over $60 \mathrm{~s}$ duration is shown here. The next three figures show details of the radar antenna sweep near $35 \mathrm{~s}$ with increasingly finer temporal resolution.

geographic features. Some of the structure close to the main beam of radar is shown in Fig. 3.2. This structure is seen to change with every capture and even within the captures. Further details can be observed by looking at the waveform in finer detail. Fig. 3.3 shows the main lobe of the sweep made up of periodic pulses with period $1 \mathrm{~ms}$, and Fig. 3.4 shows the envelope of one such pulse which has a duration of approximately $1 \mu \mathrm{s}$.

\subsection{Spectrograms and their projections onto the time and fre- quency axes}

In this section, we further describe the spectrograms that will be used extensively for the remainder of this chapter and the projection of these spectrograms onto the time- and frequency-axes. Fig. 3.5 shows a spectrogram of a signal measured, by use of the omni-direction antenna, when little or no radar signals are present. A plot of time vs. power, aggregated over all frequencies is shown at left. This aggregation is found by finding the maximum frequency bin for each time slot. We refer to this plot as the frequency-aggregated spectrogram. Similarly, a plot of power vs. frequency, aggregated over all time is shown at bottom. We refer to this plot as the time-aggregated spectrogram. Dotted lines in this plot show the typical threshold $(-76.7 \mathrm{dBm})$ for retaining the raw IQ 


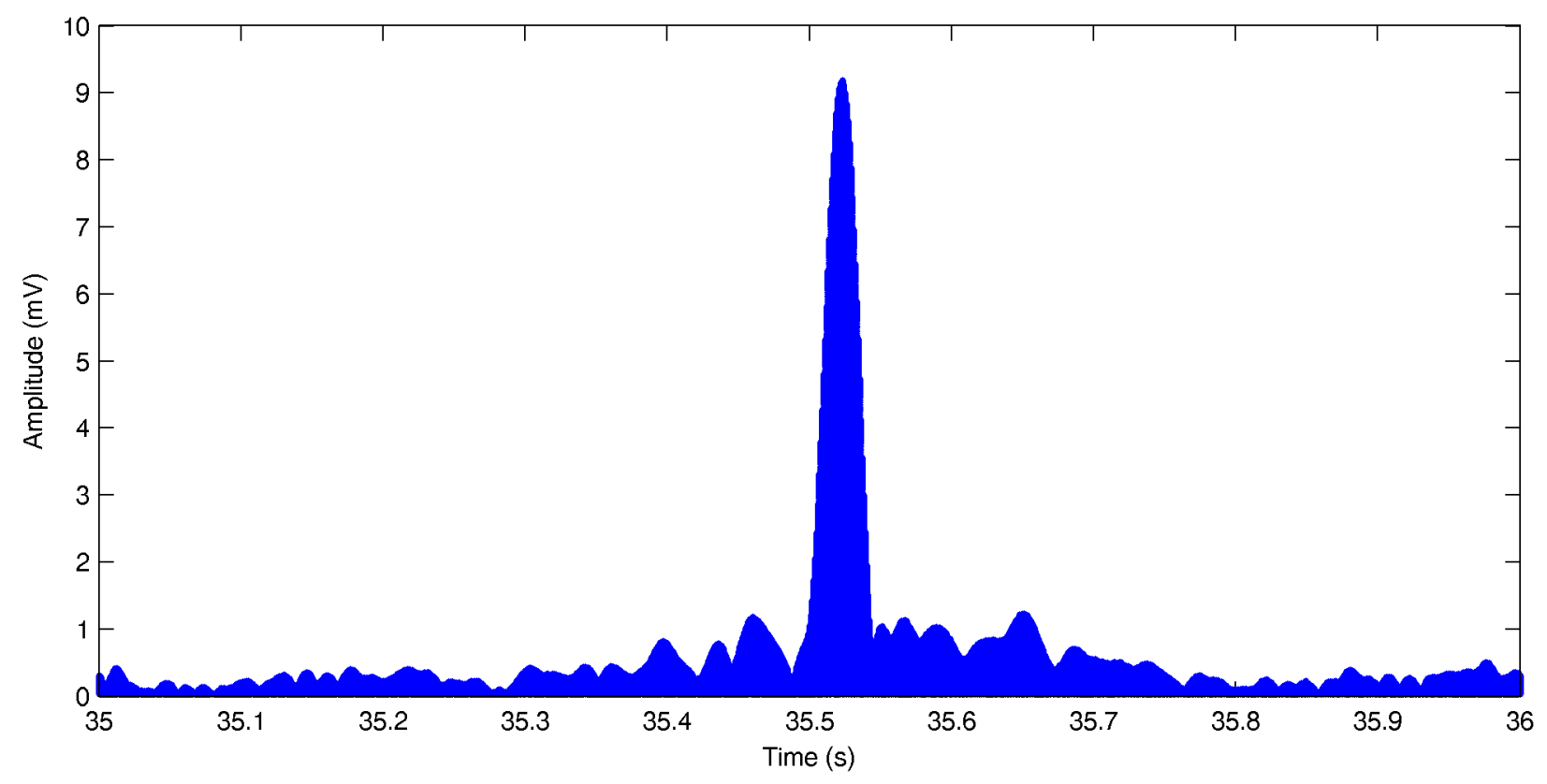

Figure 3.2: A 1 s duration sub-epoch of the SPN-43 radar capture of Fig. 3.1 showing detail in the time interval in which the main radar beam passes over the measurement system antenna.

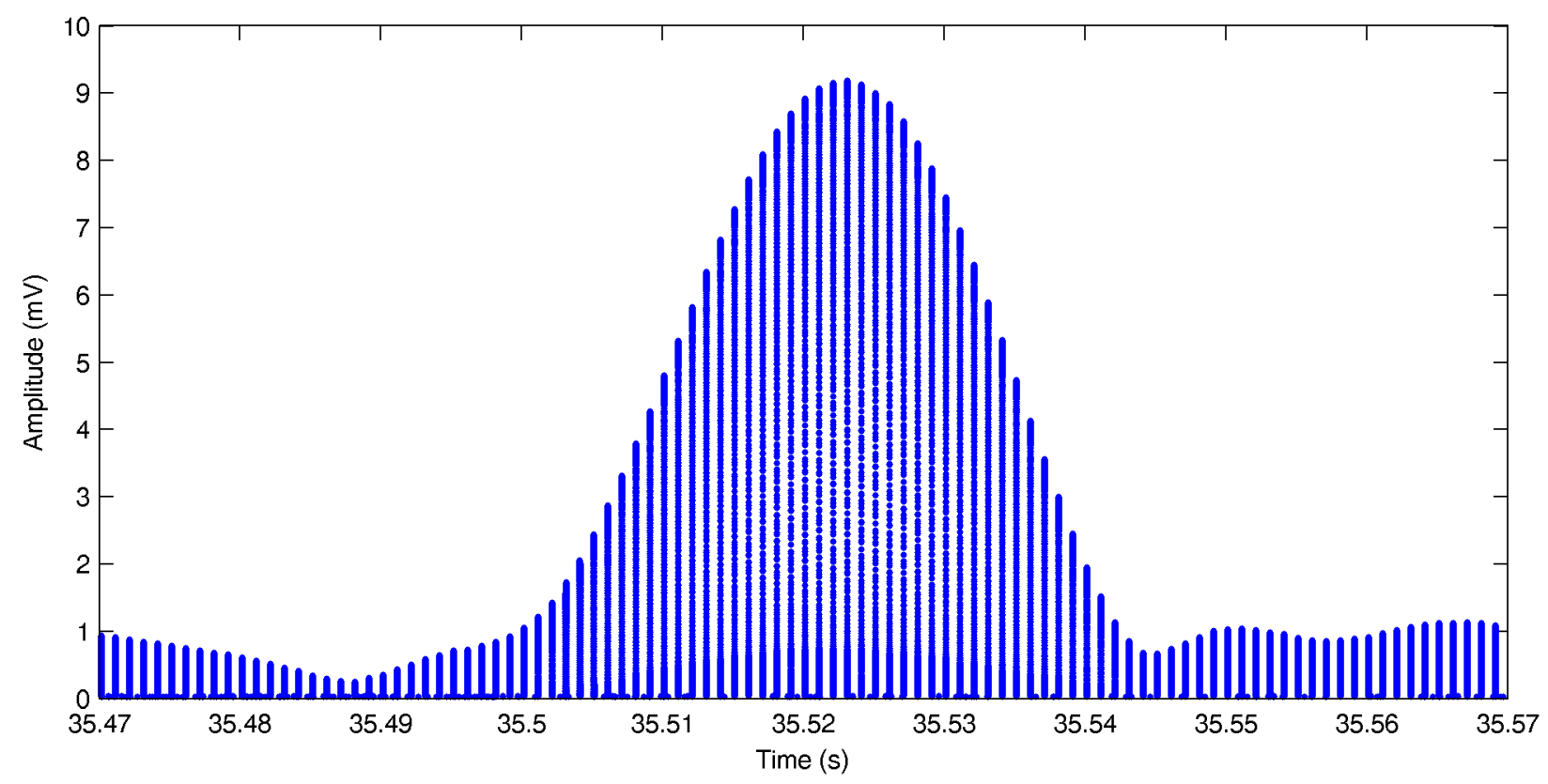

Figure 3.3: A $100 \mathrm{~ms}$ duration sub-epoch of the SPN-43 radar capture of Fig. 3.1 showing detail in the time interval in which the main radar beam passes over the measurement system antenna. Here individual pulses are resolved. 


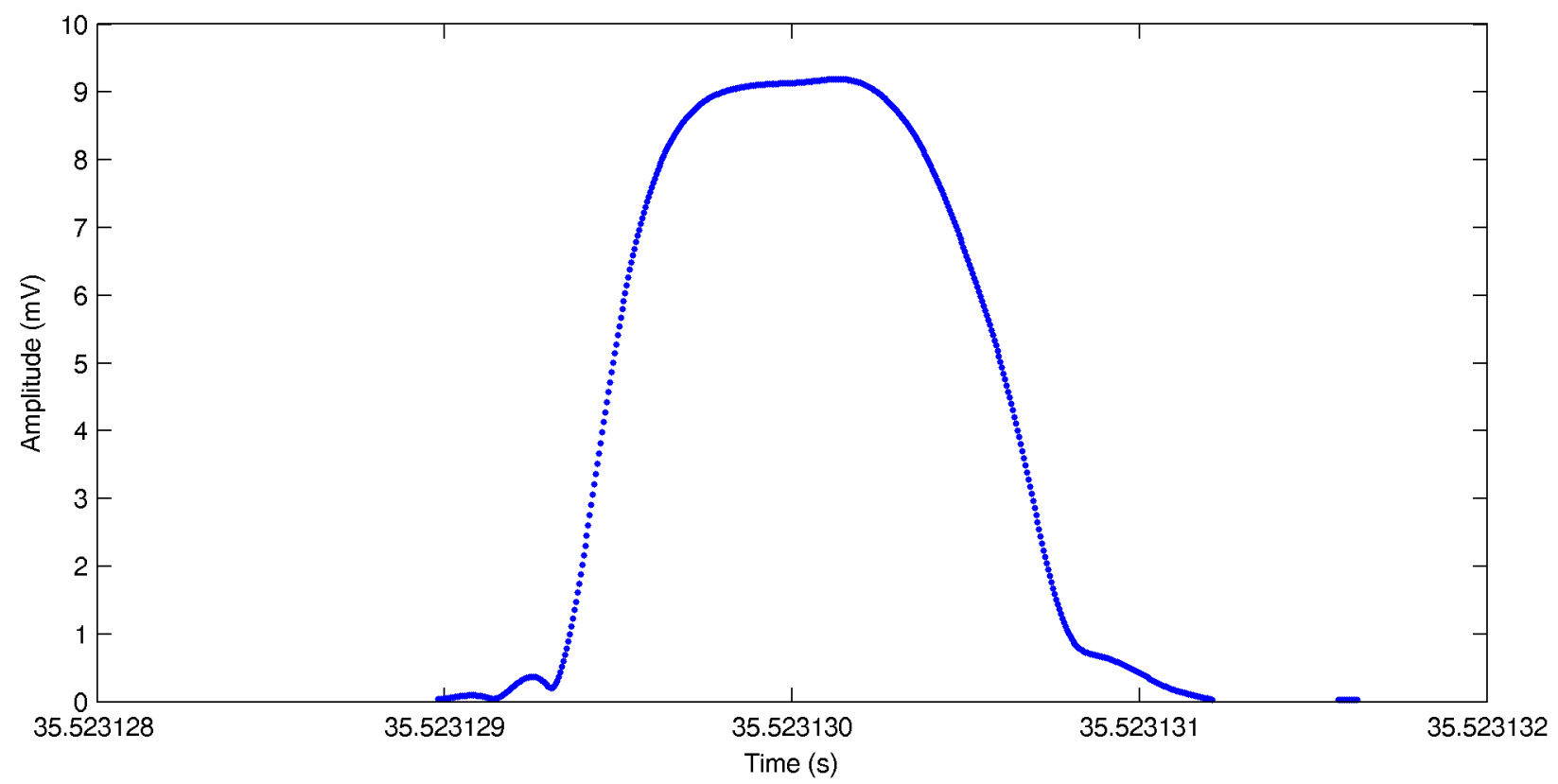

Figure 3.4: A $4 \mu$ s duration sub-epoch of the SPN- 43 radar capture of Fig. 3.1 showing detail in the time interval in which the main radar beam passes over the measurement system antenna. Here detail in an individual pulse is clearly resolved.

data and the frequency range (3522 MHz to $3673 \mathrm{MHz}$ ) over which the signal power is compared to this threshold.

Two scales are shown on the time-aggregated spectrogram: maximum power on the left and noise equivalent power (NEP) on the right. The maximum power is referenced to the output of the antenna. It is computed by converting the peak-hold frequency-domain amplitude in volts to power in decibels (assuming $50 \Omega$ impedance) referenced to one milliwatt $(\mathrm{dBm})$, subtracting the RF path gain from the output of the antenna to the input of the VST, and adding a VST calibration factor of $1.7 \mathrm{~dB}$.

The NEP is the power spectral density $(\mathrm{dBm} / \mathrm{Hz})$ one would measure if the input signal was additive white Gaussian noise. We calculate the NEP because this is the scale commonly used by analog spectrum analyzers and can be used to quantify the sensitivity of our measurement system. It is computed by converting the peak-hold frequency-domain amplitude in volts to power in decibels referenced to one milliwatt $(\mathrm{dBm})$, subtracting the RF path gain from the output of the antenna to the input of the VST, adding the $1.7 \mathrm{~dB}$ VST calibration factor, converting from peak power over the $0.455 \mathrm{~s}$ epoch to average power, ${ }^{1}$ and subtracting the effective noise bandwidth of the time-domain window used in computing the discrete Fourier transform $(53.8 \mathrm{dBHz})$.

\footnotetext{
${ }^{1}$ The ratio of the peak to average power, assuming additive white Gaussian noise and where the peak is obtained over $1.28 \times 10^{5}$ independent complex Gaussian envelope samples, is approximately $10.9 \mathrm{~dB}$.
} 
The noise at frequencies below $3477 \mathrm{MHz}$ and above $3677 \mathrm{MHz}$ falls due to the anti-aliasing filter inside the VST. The anti-aliasing filter plus the pre-selector proved to be effective in eliminating signal images due to aliased out of band signals, except when those signals were quite large, e.g., Fig. 3.15. In these cases it is visually clear when there is an image present above $\sim 3677$ $\mathrm{MHz}$ due to strong signals present below $3464.5 \mathrm{MHz}$.

The peak in Fig. 3.5 at $3577 \mathrm{MHz}$ for all times is present because of the direct downconversion architecture of our VST. It is due to LO energy leaking between the LO port and the IF port of the mixers used for IQ demodulation. For display purposes, the actual LO tone, which is typically about $10 \mathrm{~dB}$ higher than the surrounding pedestal, has been suppressed by setting its power equal to the average of the powers in the FFT bins on either side of the LO tone.

\subsection{Qualitative behavior of SPN-43 in normal operation}

The spectrogram of Fig. 3.6 shows typical behavior of a SPN-43 radar, as expected from [5], with a slightly smaller antenna rotation period of approximately $3.8 \mathrm{~s}$. The pulses display a shoulder towards lower frequency, as is commonly observed with conventional magnetron radar sources $[14,15]$. The region between rotations, which is nominally in the antenna back lobes, exhibits power levels that are at least $10 \mathrm{~dB}$ above our receiver's noise floor. Possible explanations for the structure between rotations include reflections from the ship's superstructure, reflections from large nearby objects, such as large ships, reflections from geographic features near the receiver, and back lobes of the antenna itself. The structure of the measured between-rotation signals evolves with time in all of our measured spectrograms. In this case, the peak sweep powers vary slowly and by about $4.8 \mathrm{~dB}$.

The spectrogram of Fig. 3.7 shows the received radar signal undergoing $19.3 \mathrm{~dB}$ of fading with some large structure between the main peaks. Thus, ESC detectors may need to be robust against a range of fading channels.

During normal operations, SPN-43 emitters are assigned an operation frequency so as not to interfere with other SPN-43 emitters. Figs. 3.8 and 3.9 show observed examples of simultaneous operation of two SPN-43 systems. Simultaneous operation of two SPN-43 systems was also observed at Point Loma [1]. 


\subsection{Observed SPN-43 anomalous behavior}

We expected the SPN-43 to operate at a single frequency so as to not interfere with other users of the spectrum that have a fixed spectrum allocation [16]. Fig. 3.10 shows a counter example where the frequency of a SPN-43 is apparently being tuned in frequency at a constant rate between the beginning of the emission and about $57 \mathrm{~s}$, at which time the tuning reverses. Similar tuning behavior was also observed at Point Loma [1].

In Fig. 3.11 we see the radar turning on, where the initial frequency and rotation period are both slightly higher than the quiescent values. Fig. 3.12 shows another example of this phenomenon. Frequency changes at the beginning of detected emission may be due to intentional tuning or to instability in the radar system just after being turned on. Rotation period changes may be due to the radar rotation startup and proceeding to the quiescent rotation rate. Similar instability after start of emission was also observed at Point Loma [1].

\subsection{Adjacent-band emitters}

It was concluded in [8] that adjacent-band radar, in particular the Radar 3 of [10], could generate significant emissions into the $3.5 \mathrm{GHz}$ band, as shown in Fig. 3.13. In that figure, the emissions into the $3550 \mathrm{MHz}$ to $3650 \mathrm{MHz}$ band can be roughly $50 \mathrm{~dB}$ below the power emitted into the primary $3100 \mathrm{MHz}$ to $3500 \mathrm{MHz}$ band. We confirmed this conclusion in numerous measurements, e.g., Fig. 3.14, where the power in the $3550 \mathrm{MHz}$ to $3650 \mathrm{MHz}$ band is approximately $10 \mathrm{~dB}$ above the system noise floor and less than only $40 \mathrm{~dB}$ lower in power than the signal in the $\sim 3478 \mathrm{MHz}$ to $3498 \mathrm{MHz}$ band. Although the observable band of our recording equipment was limited to $3477 \mathrm{MHz}$ to $3677 \mathrm{MHz}$, we were able to observe spectra over the entire $3 \mathrm{GHz}$ to $4 \mathrm{GHz}$ band with similar characteristics to those reported in [8] by use of a separate observation system ${ }^{2}$ and verify concurrent signals in the $3100 \mathrm{MHz}$ to $3500 \mathrm{MHz}$ band and broadband signals in the $3.5 \mathrm{GHz}$ band. Furthermore, ships carrying Radar 3 were commonly observed visually during and in the vicinity of our measurements. We therefore conclude that the broadband signals observed in the $3550 \mathrm{MHz}$ to $3650 \mathrm{MHz}$ band are indeed emanating from Radar 3.

Note that changing the preselector to have better low frequency out-of-band rejection reduced or eliminated aliasing issues (compare to Fig. 3.15) and reduced the possibility of unobserved out-of-band emissions saturating the VST, but significant out-of-band emissions from the adjacentband Radar 3 remain.

Broadband emissions from Radar 3 were often observed simultaneously with Radar 1 (SPN-

\footnotetext{
${ }^{2}$ Consisting of an antenna, LNA, and analog spectrum analyzer.
} 
43) during the measurement campaign, for example in Figs. 3.14 and 3.16, where a SPN-43 at $3570 \mathrm{MHz}$ is obscured, showing that Radar 3 emissions into the $3.5 \mathrm{GHz}$ band could confound SPN-43 detection. Further examples were observed at Point Loma [1]. 

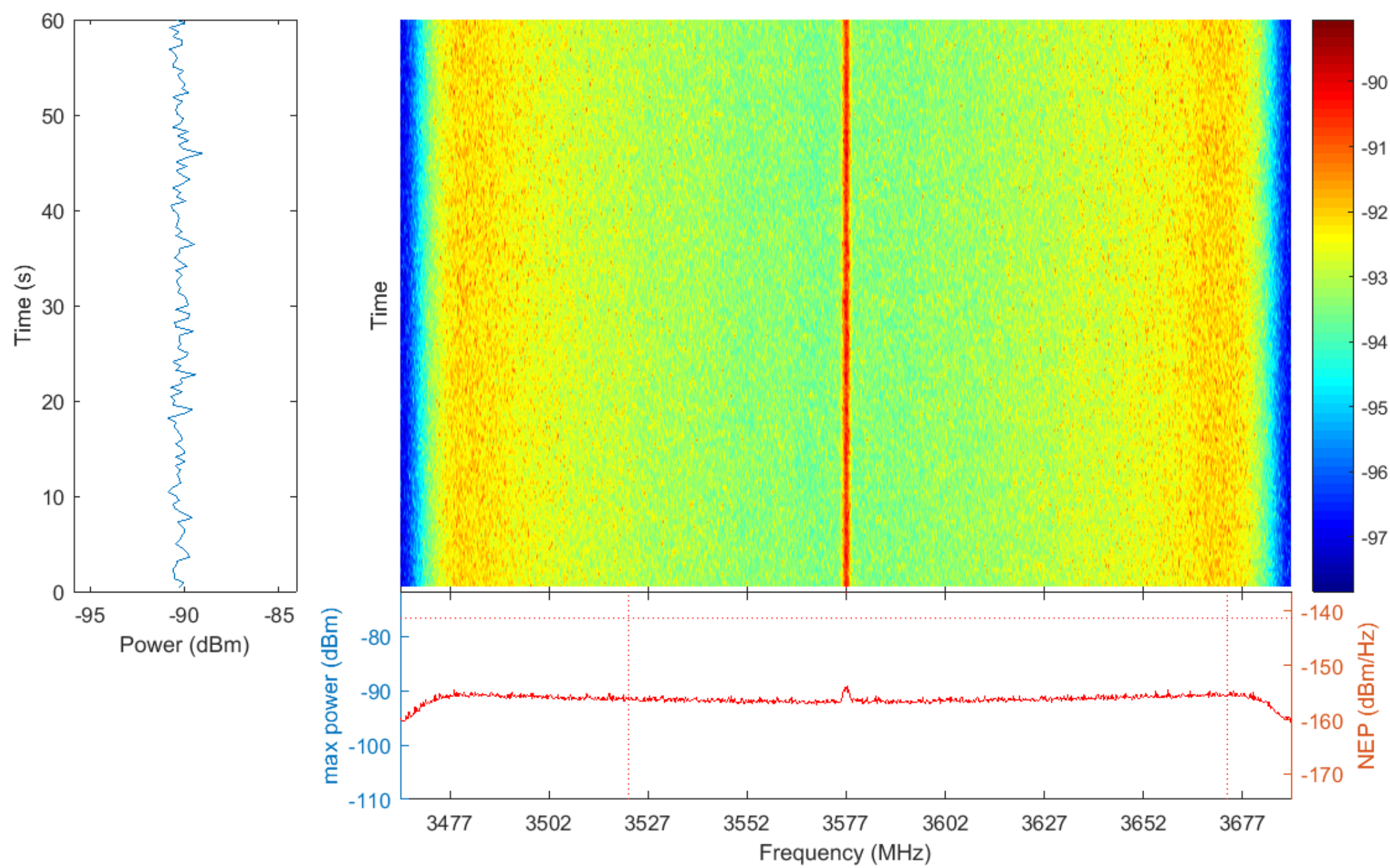

Figure 3.5: Spectrogram (colored surface plot) assembled from spectral metadata, along with the frequency-aggregated signal plotted on the left and the time-aggregated signal below. Units for the time-aggregated signal plot are described in the text. 

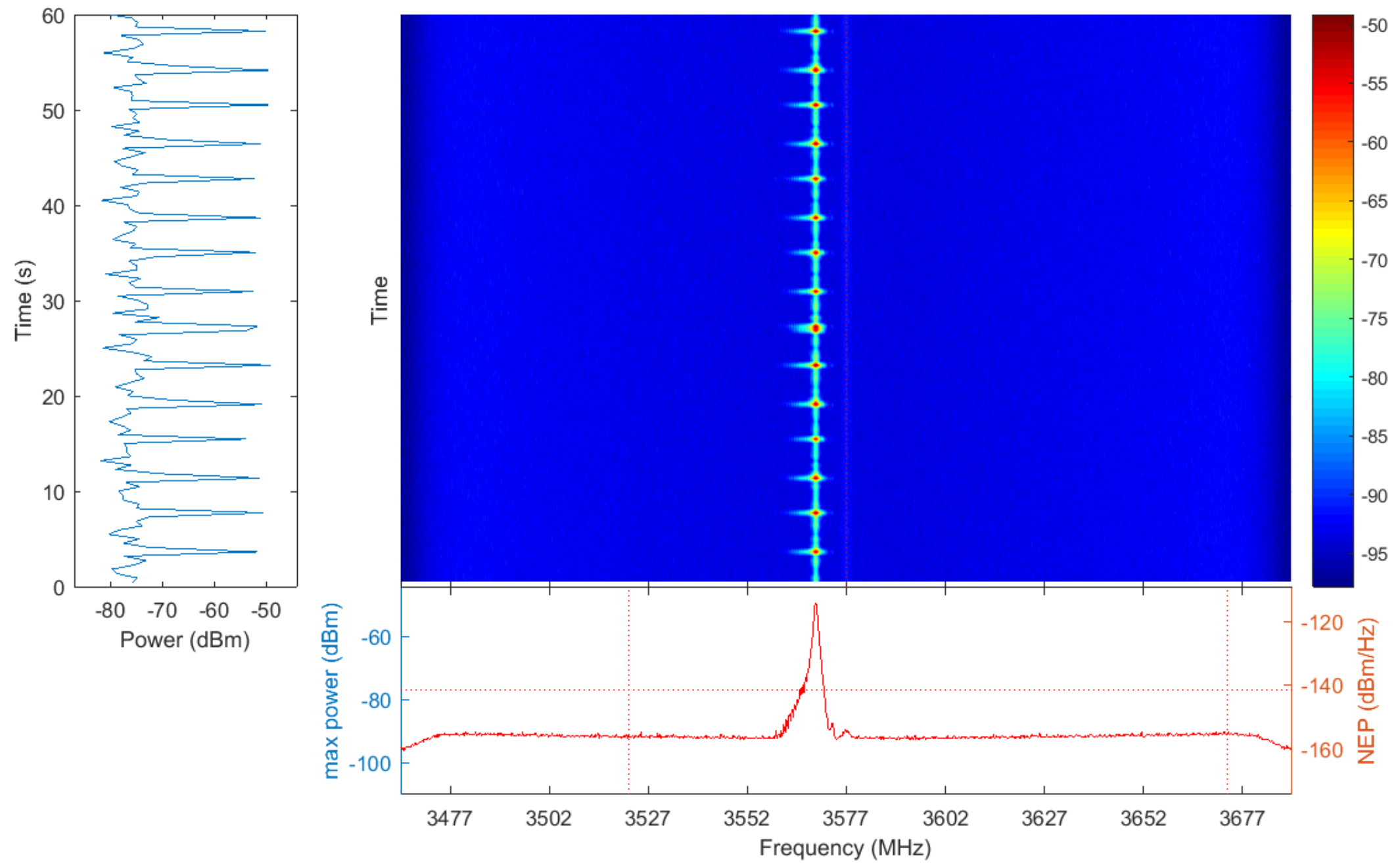

Figure 3.6: Spectrogram of a SPN-43 signal, centered near $3570 \mathrm{MHz}$, and captured with the CBS antenna. Frequency-domain pedestal on lower frequency side of peak is clearly visible. The mean peak power pooled over all rotations in this $60 \mathrm{~s}$ capture is $-51.2 \mathrm{dBm}$ in a frequency bin, referenced to the antenna output, with a spread of $4.8 \mathrm{~dB}$. 

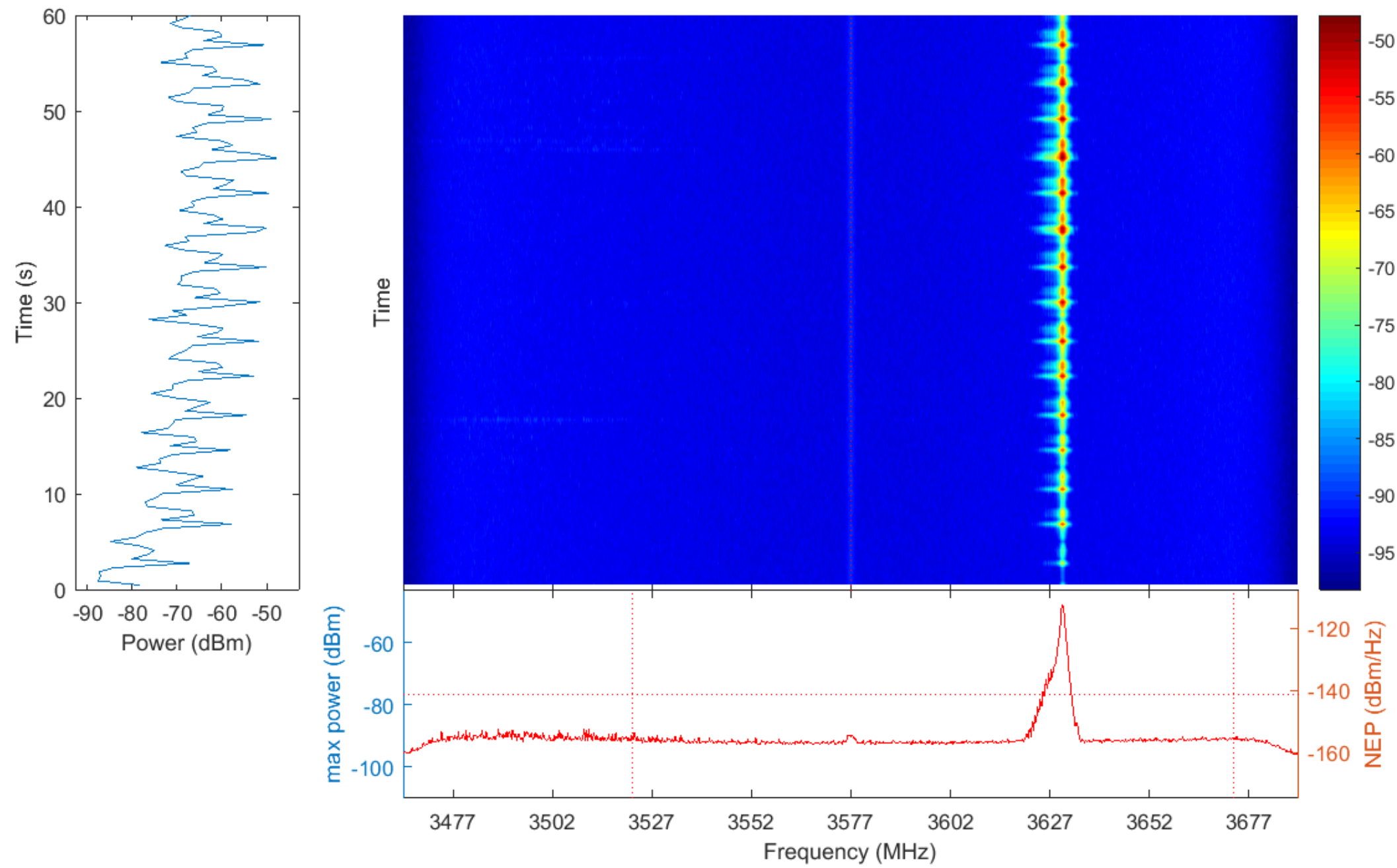

Figure 3.7: Spectrogram of a SPN-43 signal with temporal fading, captured with the CBS antenna showing spread of peak rotation power of $19.3 \mathrm{~dB}$, while the mean peak power, pooled over all rotations in this capture, is $-53.3 \mathrm{dBm}$ per frequency bin. 

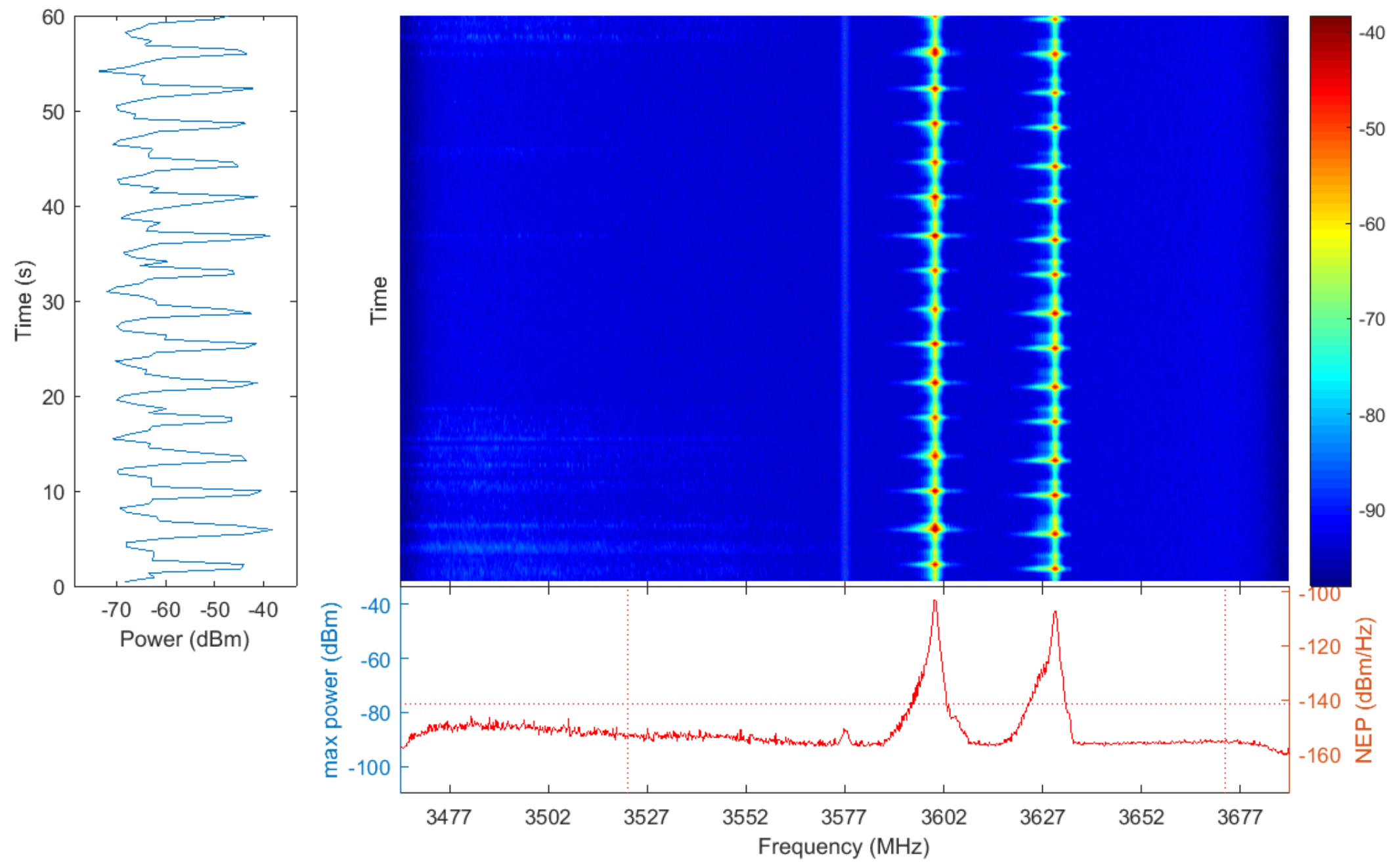

Figure 3.8: Spectrogram of SPN-43 signals at $3600 \mathrm{MHz}$ and $3630 \mathrm{MHz}$. 


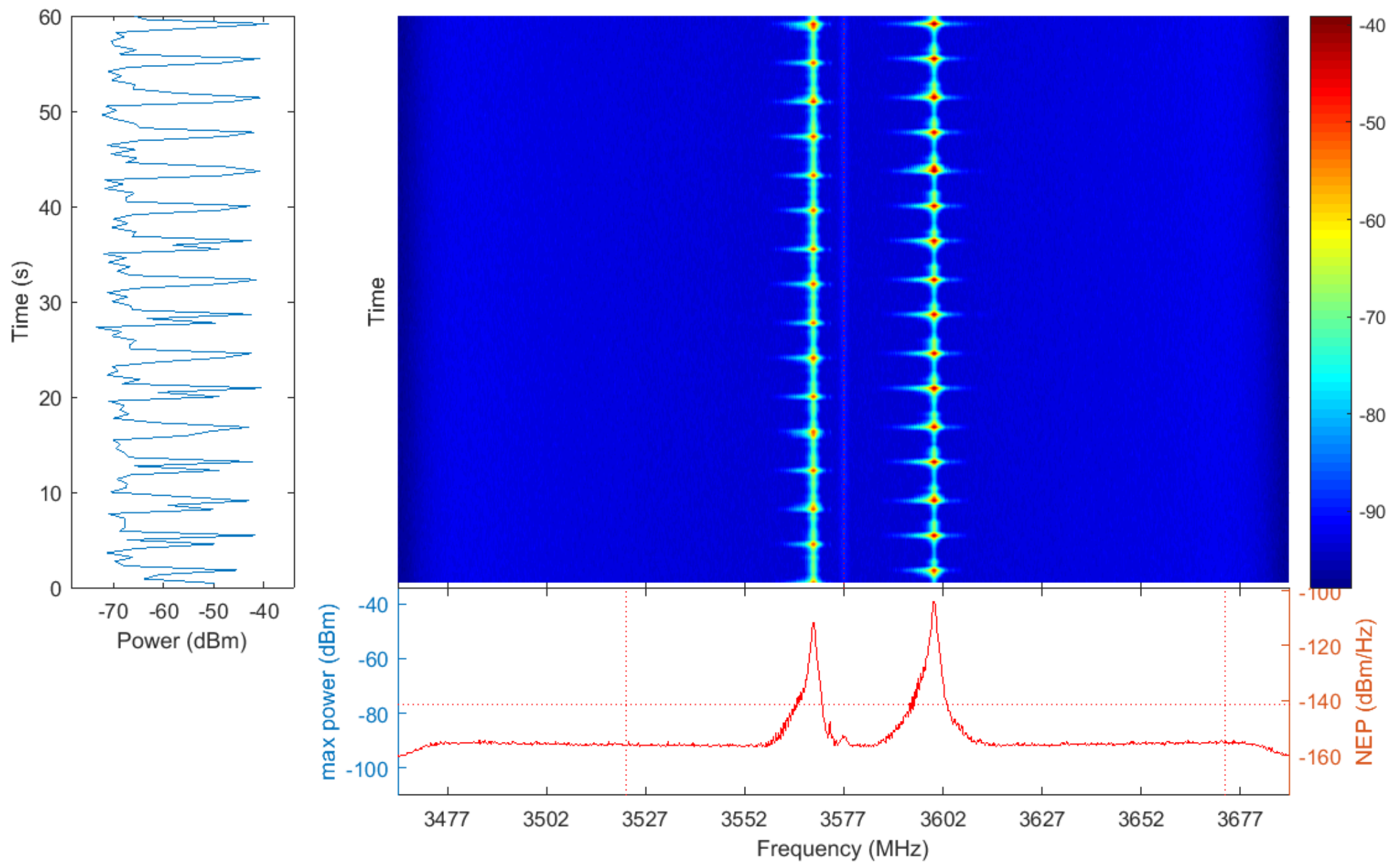

Figure 3.9: Spectrogram of SPN-43 signals at $3570 \mathrm{MHz}$ and $3600 \mathrm{MHz}$. 

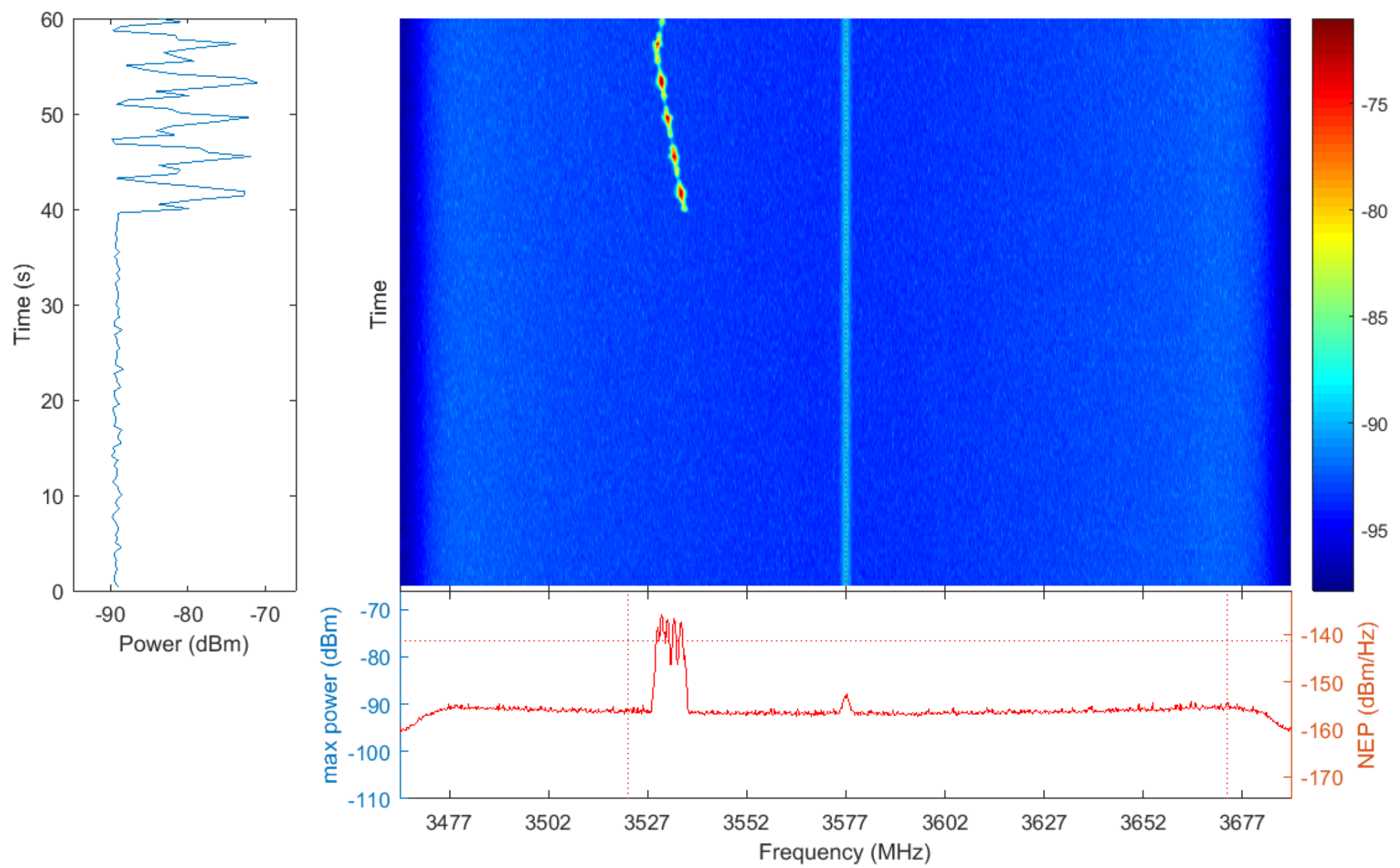

Figure 3.10: Spectrogram of a SPN-43 signal that is apparently being tuned at a rate of $0.41 \mathrm{MHz} / \mathrm{s}$. 

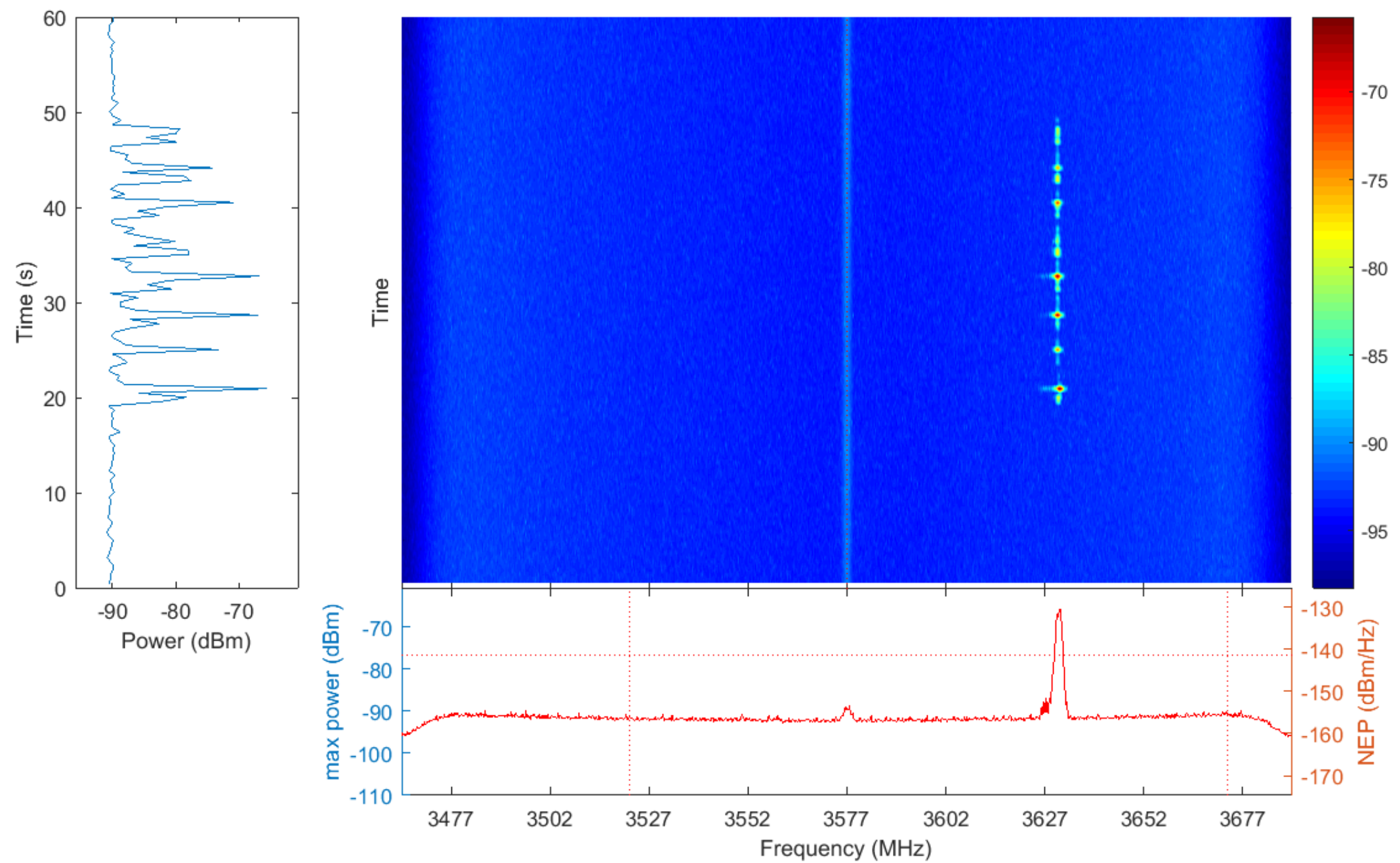

Figure 3.11: Apparent capture of SPN-43 turning on and off after about $30 \mathrm{~s}$. Note changing frequency at beginning of emissions. 

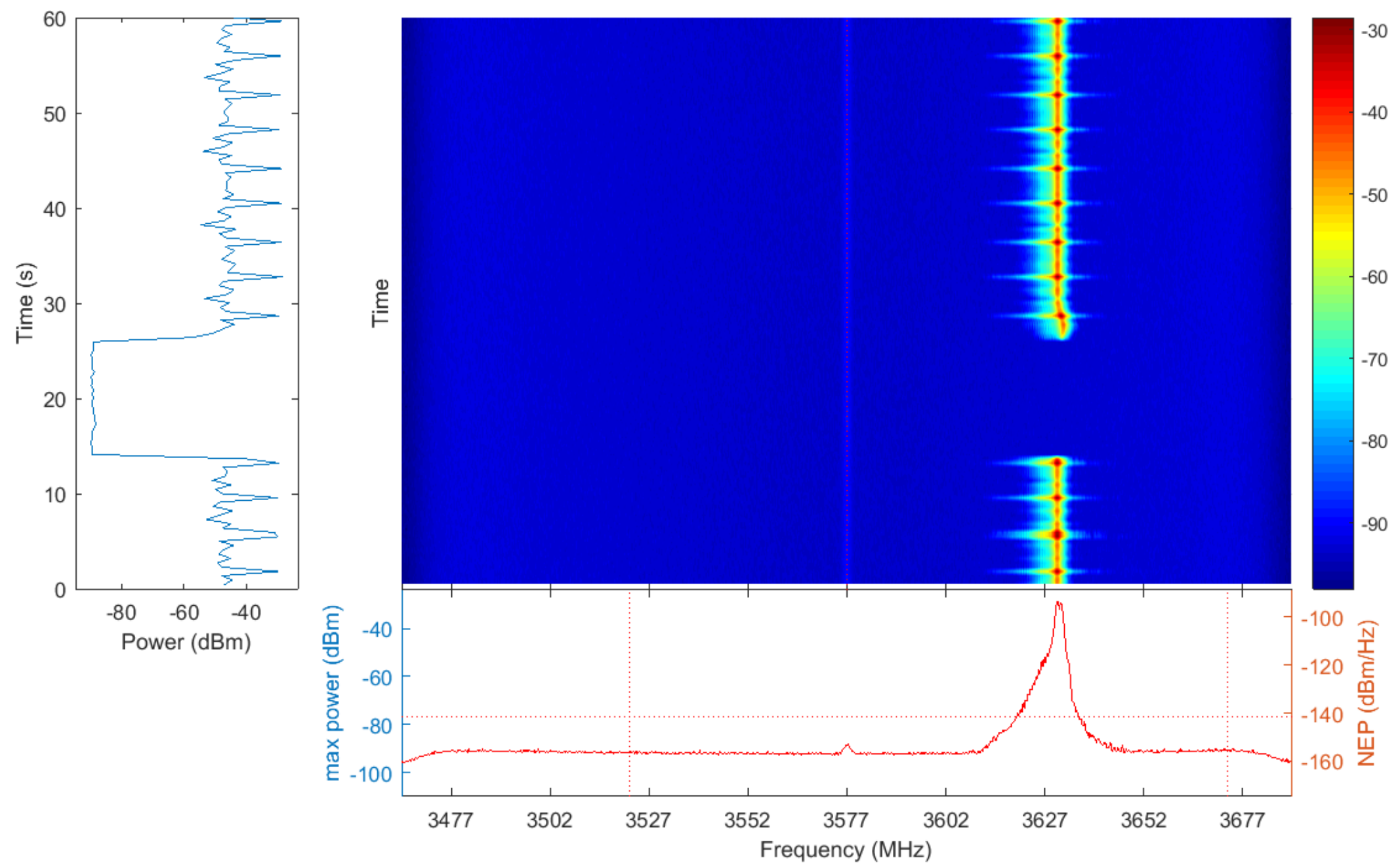

Figure 3.12: Apparent capture of SPN-43 turning off and then on after about $12.8 \mathrm{~s}$. Note changing frequency when emissions are resumed. 


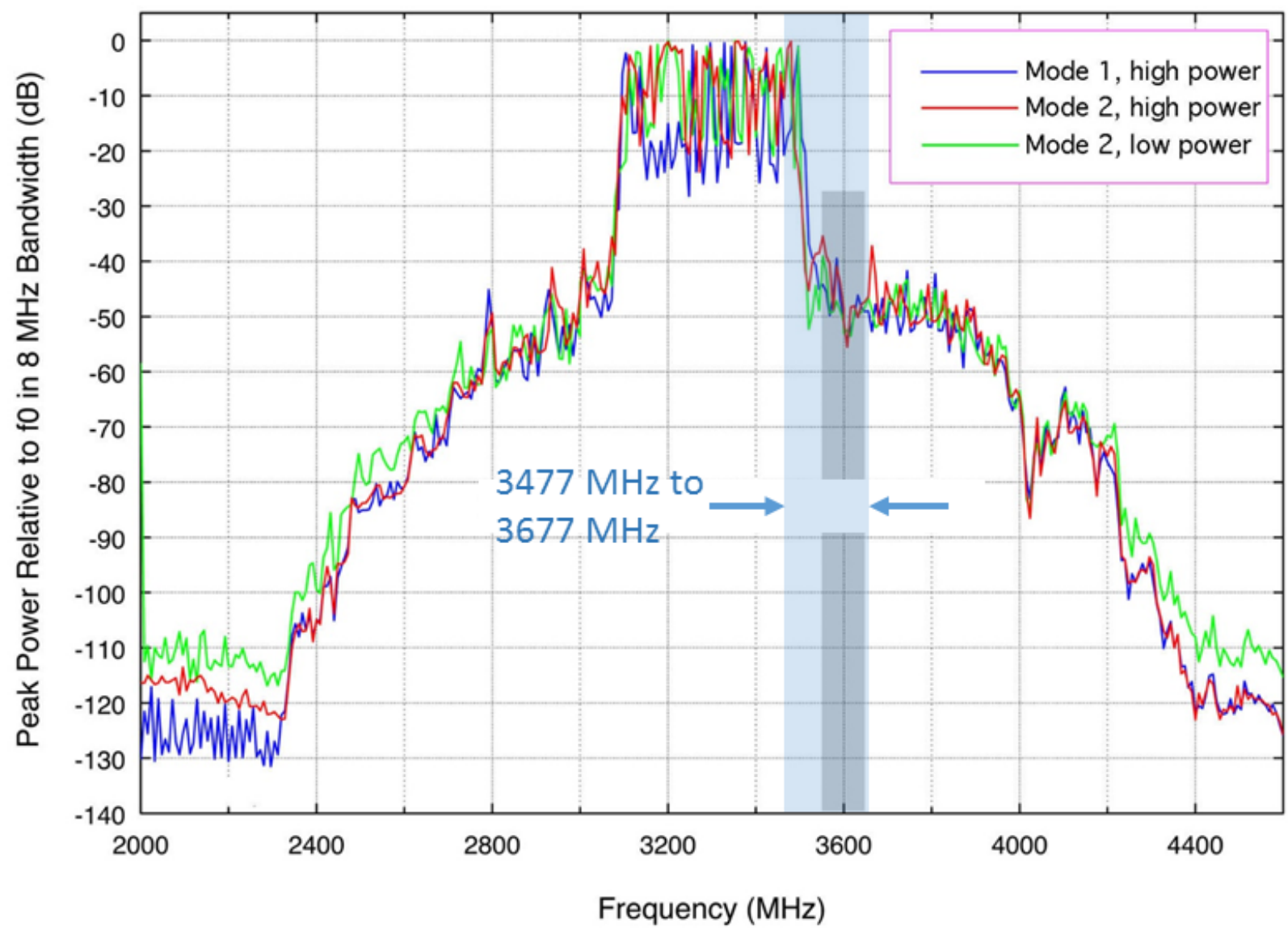

Figure 3.13: Fig. 7 of [8] showing broadband emissions of Radar 3 into the $3550 \mathrm{MHz}$ to $3650 \mathrm{MHz}$ band, shown by dark grey rectangle. Light grey rectangle is approximately the $3477 \mathrm{MHz}$ to $3677 \mathrm{MHz}$ band typically measured in this campaign, which includes some of the primary band for Radar 3. 

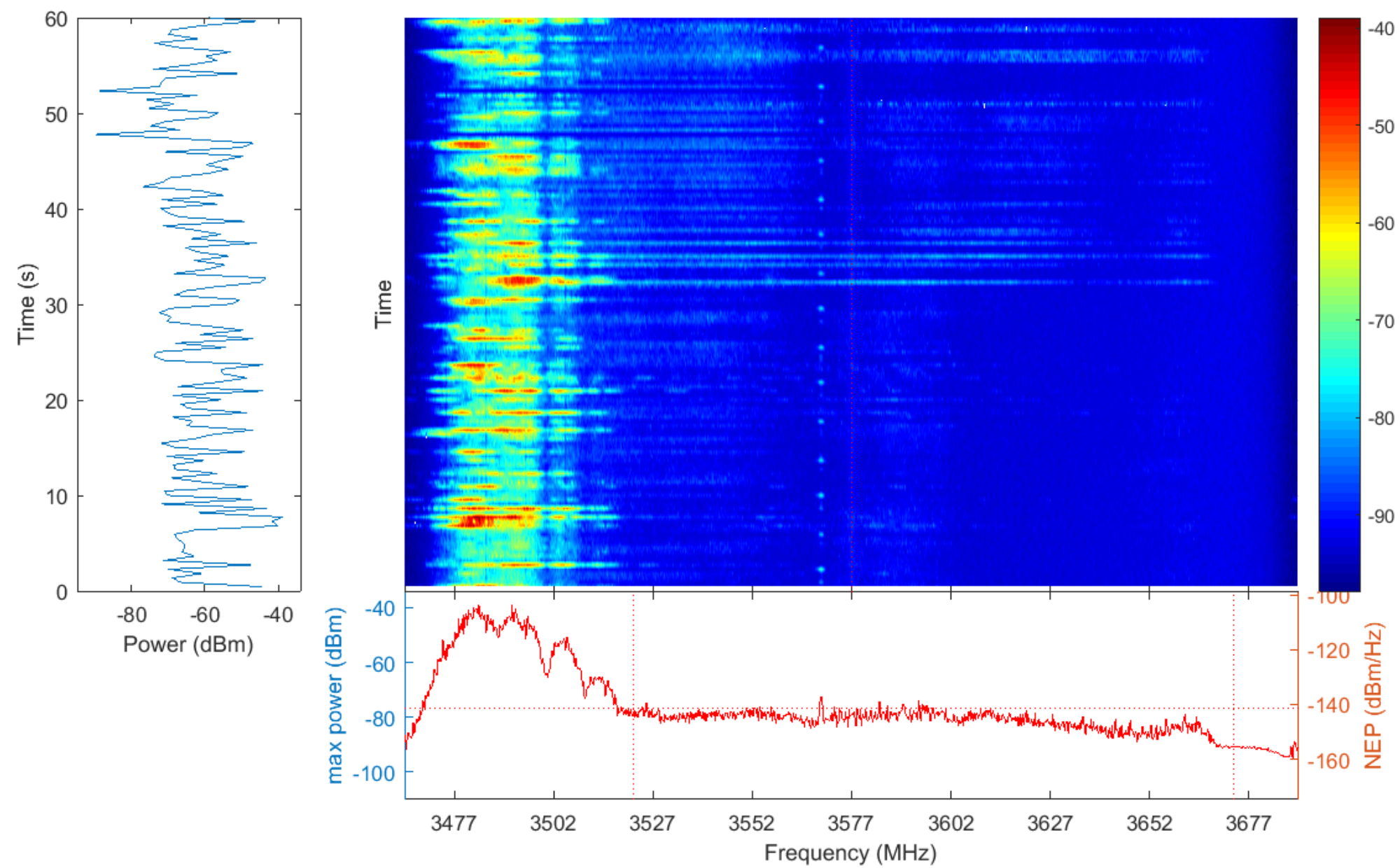

Figure 3.14: Spectrogram showing emissions from Radar 3 signal at frequencies below $3520 \mathrm{MHz}$. Note that this measurement was acquired with the omni-directional antenna and new narrow-band pre-selection filter, so that signals at frequencies lower than our measurement band do not appear as images at the right hand side of the spectrum (see Fig. 2.14). A SPN-43 emission is also apparent in this spectrogram near $3570 \mathrm{MHz}$. 

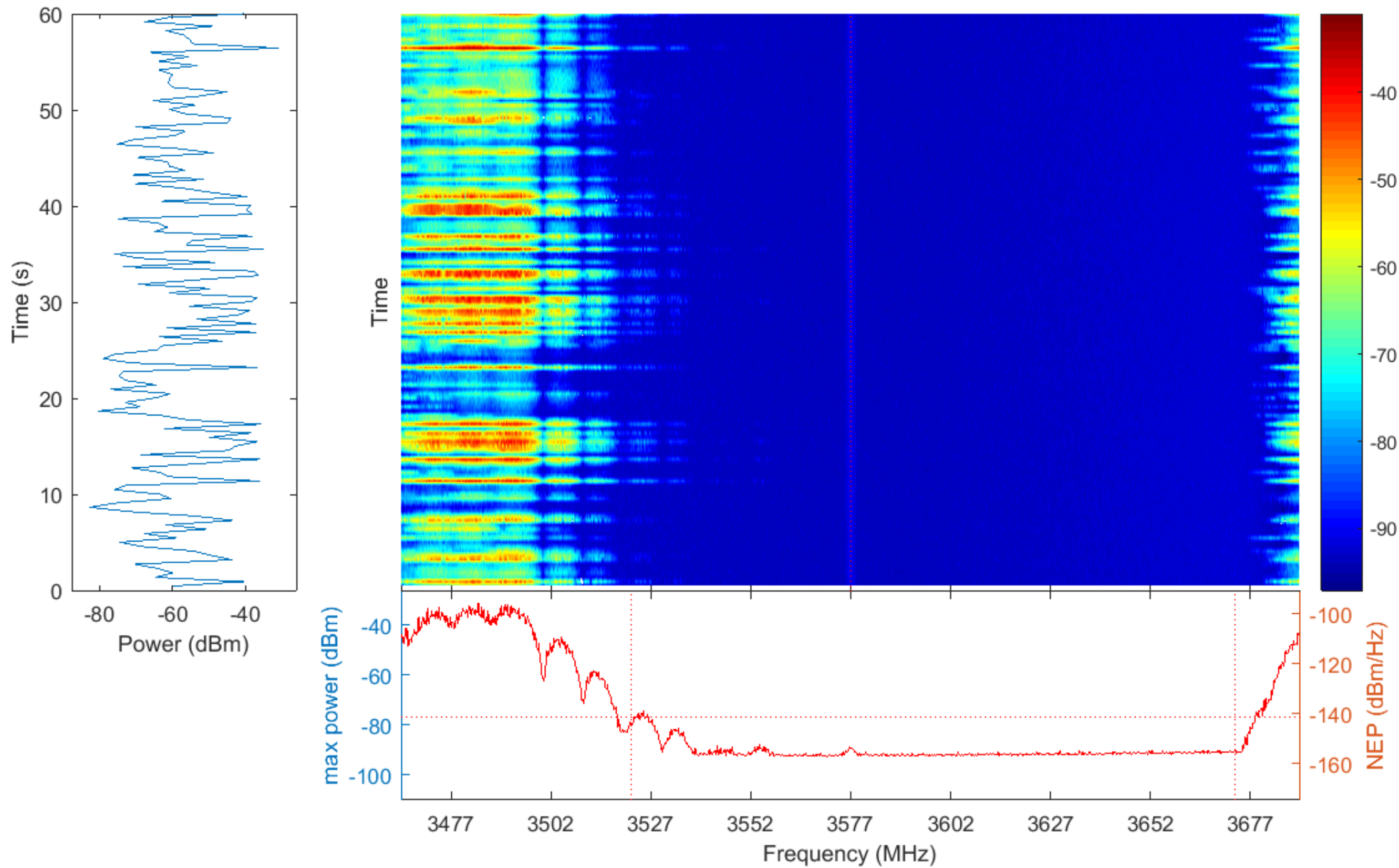

Figure 3.15: Spectrogram showing emissions from Radar 3 signal at frequencies below $3540 \mathrm{MHz}$ with aliased signal above $3670 \mathrm{MHz}$. This spectrogram was acquired by use of the CBS antenna and the old preselector filter with broader bandwidth. 

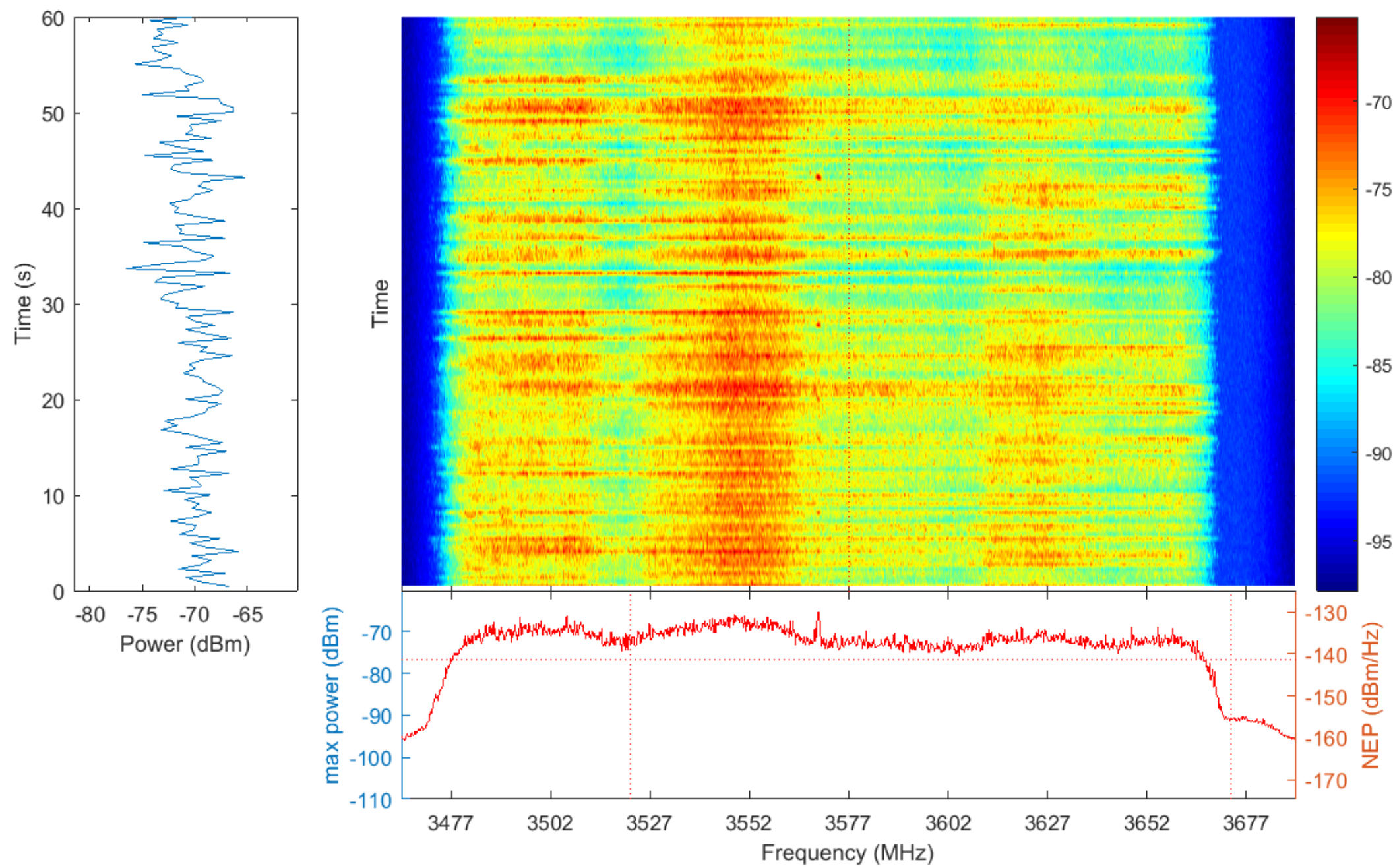

Figure 3.16: Spectrogram showing broadband noise from Radar 3 obscuring SPN-43 signal at 3570 MHz. Note that this measurement was acquired with the omni-directional antenna and narrow band pre-selection filter, so that signals at frequencies lower than our measurement band are greatly attenuated. 


\section{Chapter 4}

\section{Waveform Parameter Analysis}

This chapter presents an analysis of the radar waveforms measured in the $3550 \mathrm{MHz}$ to $3650 \mathrm{MHz}$ band. It begins with a summary of the collected data followed by a quantitative analysis of specific radar parameters.

\subsection{Summary of collected data and processing}

A total of 1473 high-fidelity, 60-s recordings were acquired over the two-month period of measurement at Fort Story. A subset of approximately 250 acquisitions was identified as having high SNR and as not containing measurable emissions from out-of-band radar systems below $3500 \mathrm{MHz}$, and this subset was used for further processing using algorithms described in [1, Appendix A].

Data processing first identifies the locations in time and frequency of the main-beam emissions of the radar in each acquisition. There are typically 15 sweeps of the main beam in each 60 -s acquisition. The maximum amplitude of each sweep and the time of its occurrence relative to the beginning of the acquisition are recorded.

Data processing then identifies the pulses within each sweep. Specifically, it selects pulses having amplitudes within $3 \mathrm{~dB}$ of the peak power of each sweep. Approximately 20 pulses are identified in each sweep. A number of parameters are then computed for each pulse, namely its maximum amplitude, center frequency, pulse duration, and the time interval between consecutive pulses.

Out of the approximately 25060 -s acquisitions that were selected for processing, a total of about 2700 antenna sweeps and 48600 pulses were identified for analysis. Table 4.1 breaks down the pulses by the antenna used for collection (omnidirectional vs. cavity-backed spiral) and the 


\begin{tabular}{|l|c|c|c|c|}
\hline & 3570 MHz & 3600 MHz & 3630 MHz & Total by Antenna \\
\hline Omni & $4.3 \%$ & $0.8 \%$ & $4.0 \%$ & $9.1 \%$ \\
\hline CBS & $24.6 \%$ & $55.2 \%$ & $11.0 \%$ & $90.9 \%$ \\
\hline Total by Frequency & $29.0 \%$ & $56.0 \%$ & $15.0 \%$ & \\
\hline
\end{tabular}

Table 4.1: Percentage of processed data by antenna and pulse center frequency

\begin{tabular}{|l|c|c|c|c|}
\hline & 3570 MHz & 3600 MHz & 3630 MHz & Total by Antenna \\
\hline Omni & 3 & 1 & 2 & 6 \\
\hline CBS & 2 & 3 & 2 & 7 \\
\hline Total by Frequency & 5 & 4 & 4 & \\
\hline
\end{tabular}

Table 4.2: Number of days over which processed data was collected, by antenna and pulse center frequency

approximate center frequency of the pulses. The vast majority of pulses (90.9\%) were captured with the CBS antenna, and of those a slight majority were observed at $3600 \mathrm{MHz}$.

The processed data was collected over 13 unique calendar days. This data is a subset of the total data collected over the course of two months. ${ }^{1}$ Table 4.2 lists the number of calendar days over which the data was collected, broken down by antenna and frequency. Although data was acquired over a similar number of days for each antenna, more of the data acquired with the CBS antenna met both the acquisition and the analysis criteria.

\subsection{Sweep parameters}

\subsubsection{Peak sweep power}

Fig. 4.1 shows a histogram of the analyzed radar sweeps by peak sweep power at the output of the measurement antenna over the entire analysis bandwidth. The peak sweep power of the analyzed data ranges from $-55 \mathrm{dBm}$ to $-15 \mathrm{dBm}$ and appears to be spread uniformly over that range. ${ }^{2}$ Considering that only pulses within $3 \mathrm{~dB}$ of the peak power are analyzed, the pulse analysis below comprises a range of over $40 \mathrm{~dB}$.

\footnotetext{
${ }^{1}$ Though the measurement system was deployed at Fort Story for a period of two months, there was downtime due to hurricanes Hermine and Matthew. Furthermore, emissions of the target radar in the absence of adjacent-band radar occurred on a fraction of the uptime.

${ }^{2}$ Note that the peak powers quoted here, measured in the time domain, are higher than their frequency-domain counterparts in Chapters 2 and 3 due to time-averaging of the approximately $1 \mu \mathrm{s}$ pulse in the 1024 -sample (4.55 $\mu \mathrm{s})$ window of the discrete Fourier transform. The difference due to pulse desensitization in the frequency domain is approximately $13 \mathrm{~dB}$, assuming a rectangular pulse shape.
} 


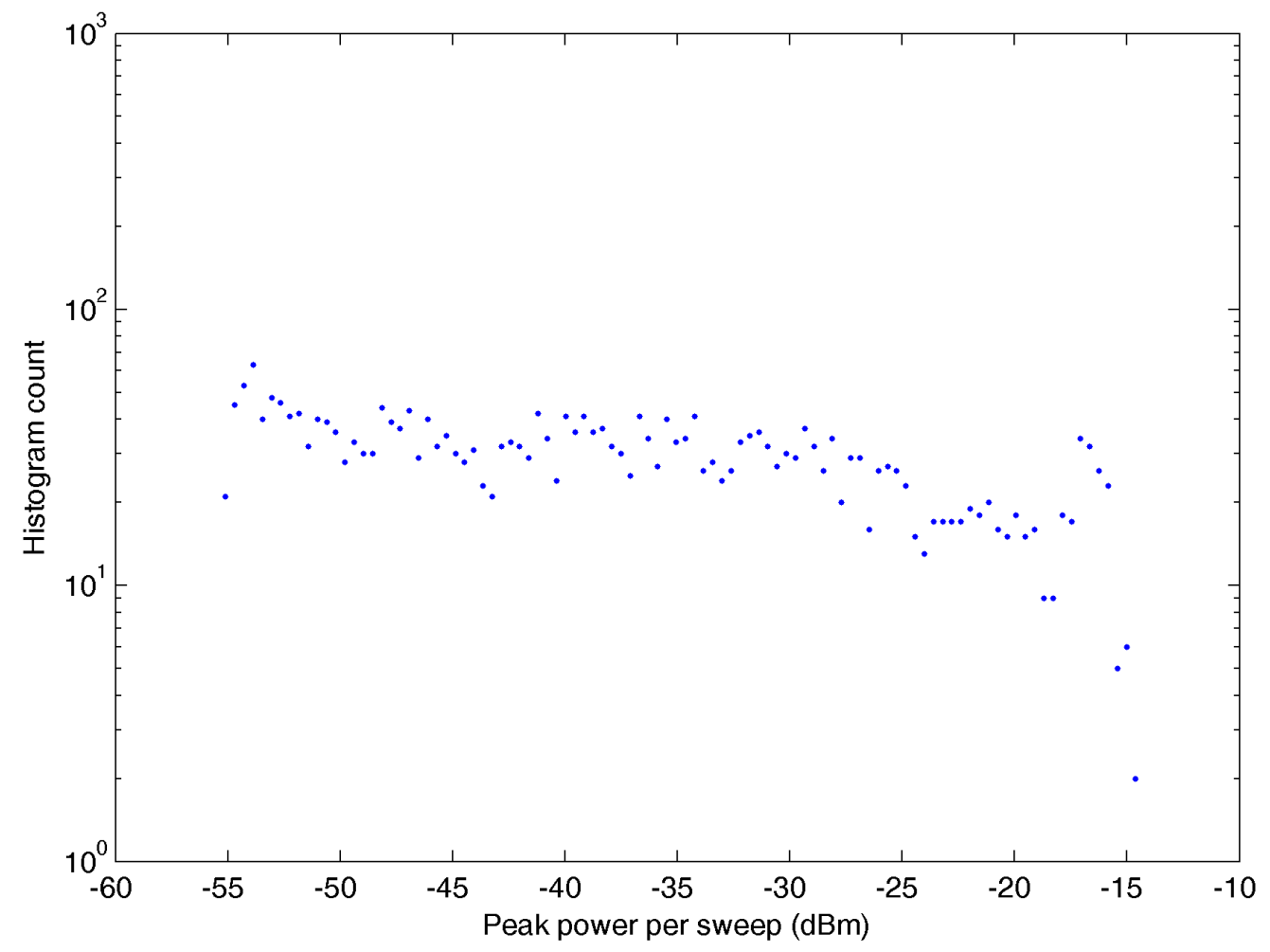

Figure 4.1: Histogram of peak power per antenna sweep

\subsubsection{Sweep interval}

The sweep interval is the time interval between successive peaks of received power due to the rotation of the radar's transmitting antenna in the azimuth plane. Fig. 4.2 plots the empirical cumulative distribution function (CDF) and complementary CDF (CCDF) of the sweep interval for all 2700 sweeps. The empirical CDF and CCDF depict the distribution of the observations from which key percentiles, including the median, can be read. Probabilities are shown on a logarithmic scale in order to better view the lower tail of the distribution via the CDF and the upper tail via the complementary CDF (one minus the CDF). For example, Fig. 4.2 shows that the median sweep interval is $3.858 \mathrm{~s}(\approx 0.14 \mathrm{~s}$ lower than given in [2]), and that $80 \%$ of the measured sweep intervals (those between the 10th and 90th percentiles) lie between $3.852 \mathrm{~s}$ and $3.907 \mathrm{~s}$. The antenna rotation rates corresponding to this $80 \%$ interval are (15.36 to 15.58$)$ rotations per minute. The dotted lines in Fig. 4.2 represent the $95 \%$ confidence bounds on the empirical data, assuming independence of the observations [17].

The upper tail of the sweep interval distribution indicates measured sweep intervals at multiples of the antenna rotation period (not shown on the scale of Fig. 4.2). For example, the 99th percentile of the measured sweep interval lies between the fourth and fifth multiples of the median sweep interval, that is, between $15.43 \mathrm{~s}$ and $19.29 \mathrm{~s}$. The heavy upper tail is indicative of signal fading 


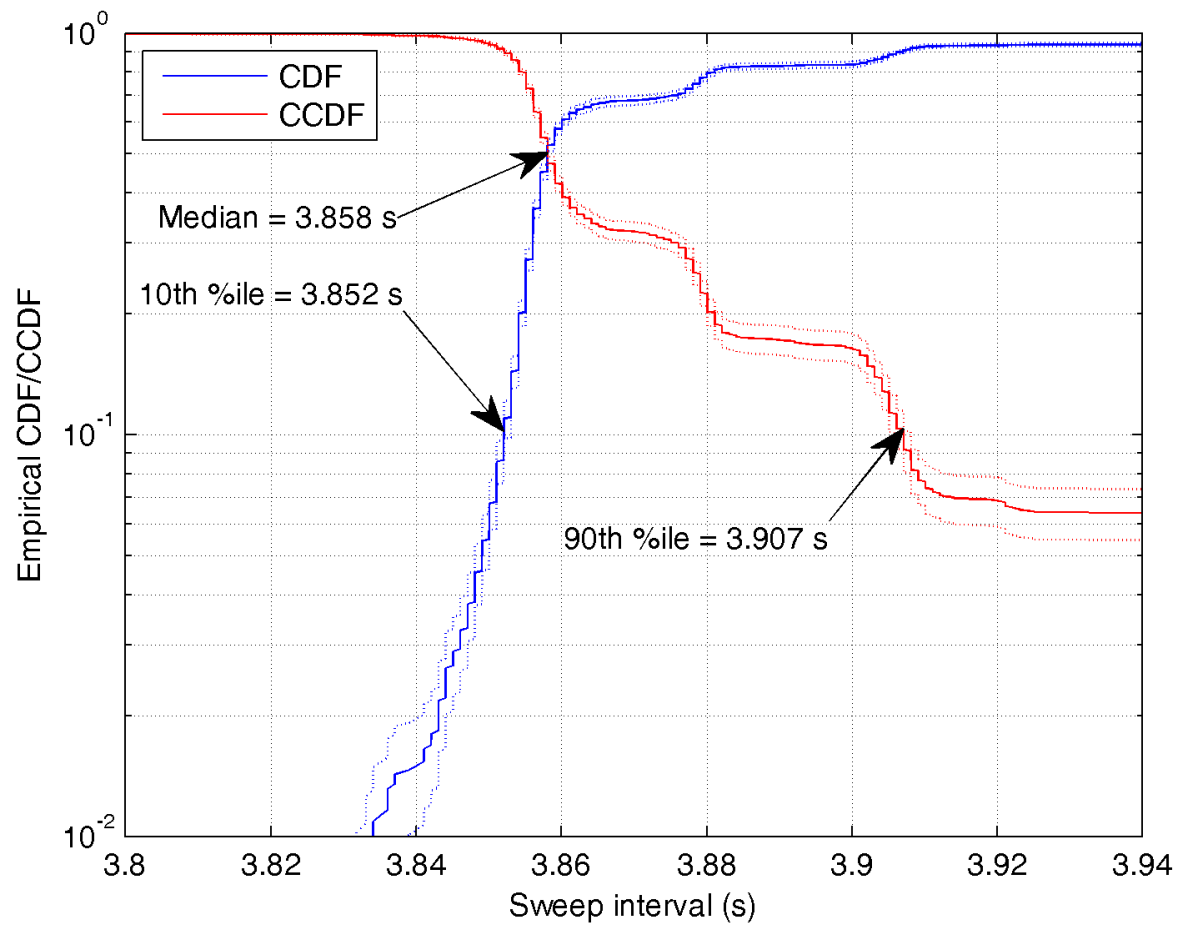

Figure 4.2: Empirical CDF and complementary CDF (CCDF) of sweep interval annotated with select percentiles (\%ile) 

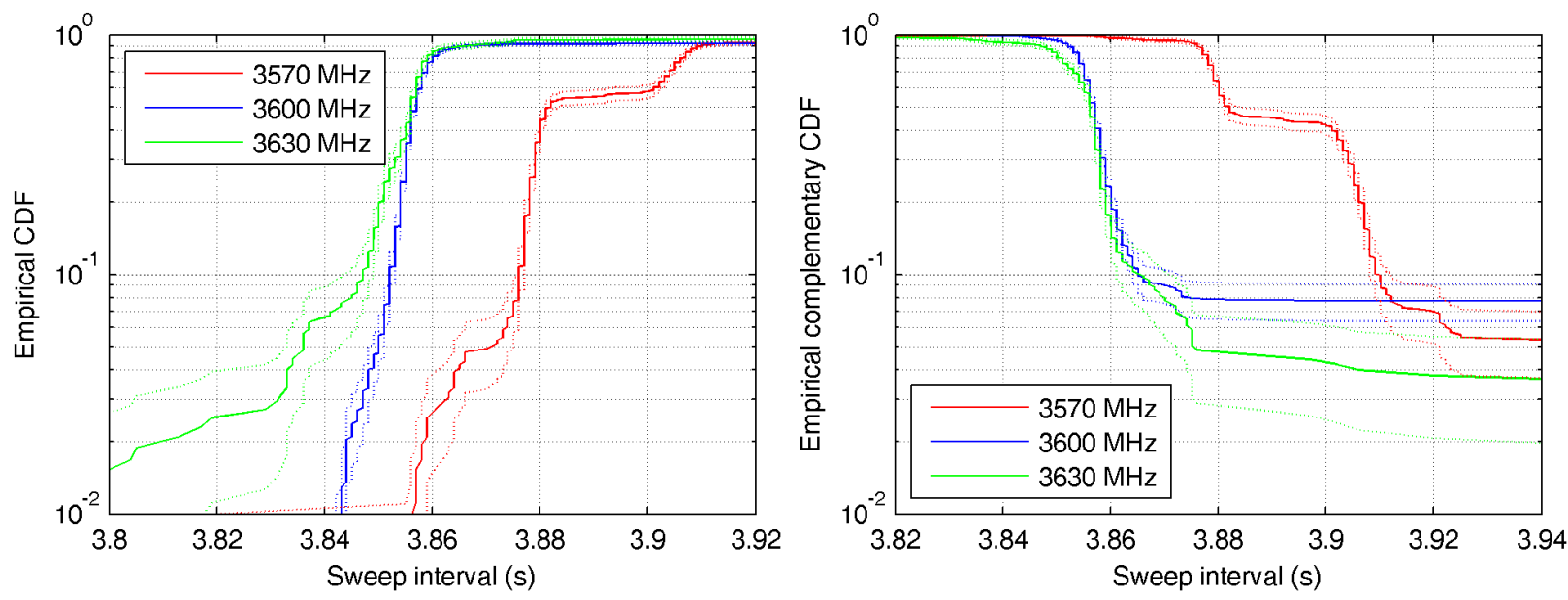

Figure 4.3: Empirical CDF and CCDF of sweep interval by center frequency

which caused some intervening sweeps to be received at power levels below the data analysis threshold for SNR.

Fig. 4.3 illustrates the empirical CDF and CCDF of the sweep interval by center frequency. The center frequency can be used as a surrogate for unique radar transmitters, assuming that each transmitter operates at a different frequency. ${ }^{3}$ Signals observed at $3570 \mathrm{MHz}$ exhibit a slightly longer antenna rotation period (about $25 \mathrm{~ms}$ longer in the median) than those observed at the other two frequencies.

\subsection{Pulse parameters}

\subsubsection{Pulse duration}

Pulse duration is defined as the time between the rising-edge and falling-edge crossings by the pulse envelope of half the maximum amplitude of the pulse (see [1, Appendix A.2] for a more detailed description). Fig. 4.4 plots the empirical CDF and CCDF of the pulse duration over all 48600 analyzed pulses. The empirical distribution indicates that the median pulse duration is $1.15 \mu \mathrm{s}(0.20 \mu \mathrm{s}$ higher than expected from [2]), and that $98 \%$ of the measured pulse durations lie between $1.05 \mu \mathrm{s}$ and $1.17 \mu \mathrm{s}$.

Fig. 4.5 illustrates the distribution of the measured pulse durations by center frequency. We observe that the median pulse duration differs by close to $0.05 \mu$ s across the three observed fre-

\footnotetext{
${ }^{3}$ As observed in [1, Section 3.5], the assumption of a one-to-one correspondence between frequency and system does not hold universally.
} 


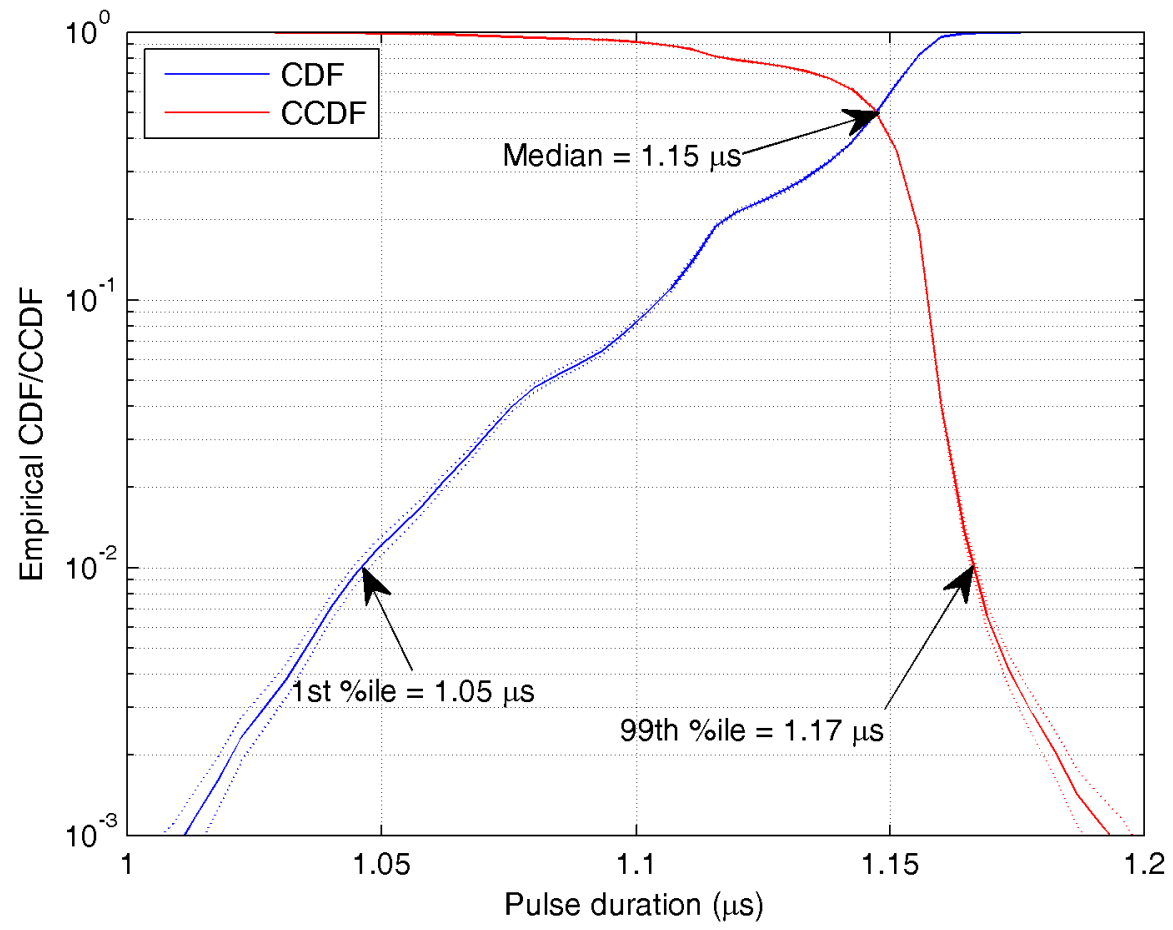

Figure 4.4: Empirical CDF and CCDF of pulse duration over all data
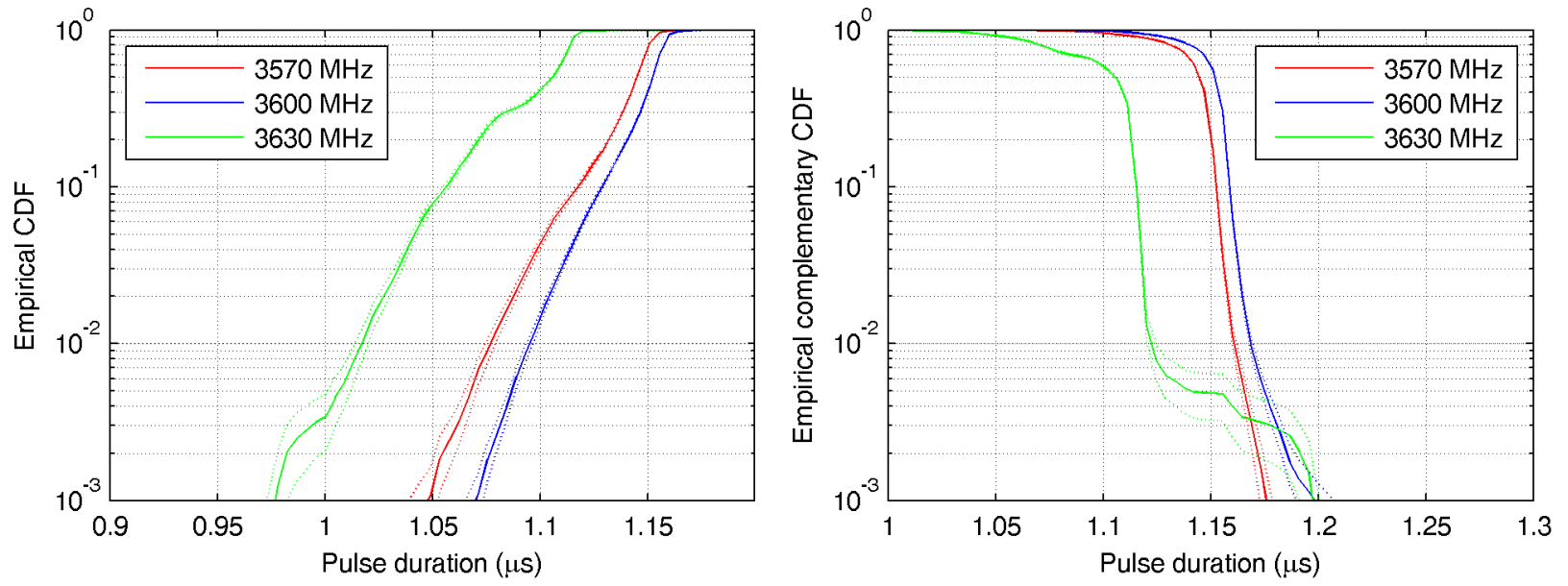

Figure 4.5: Empirical CDF and CCDF of pulse duration by center frequency 

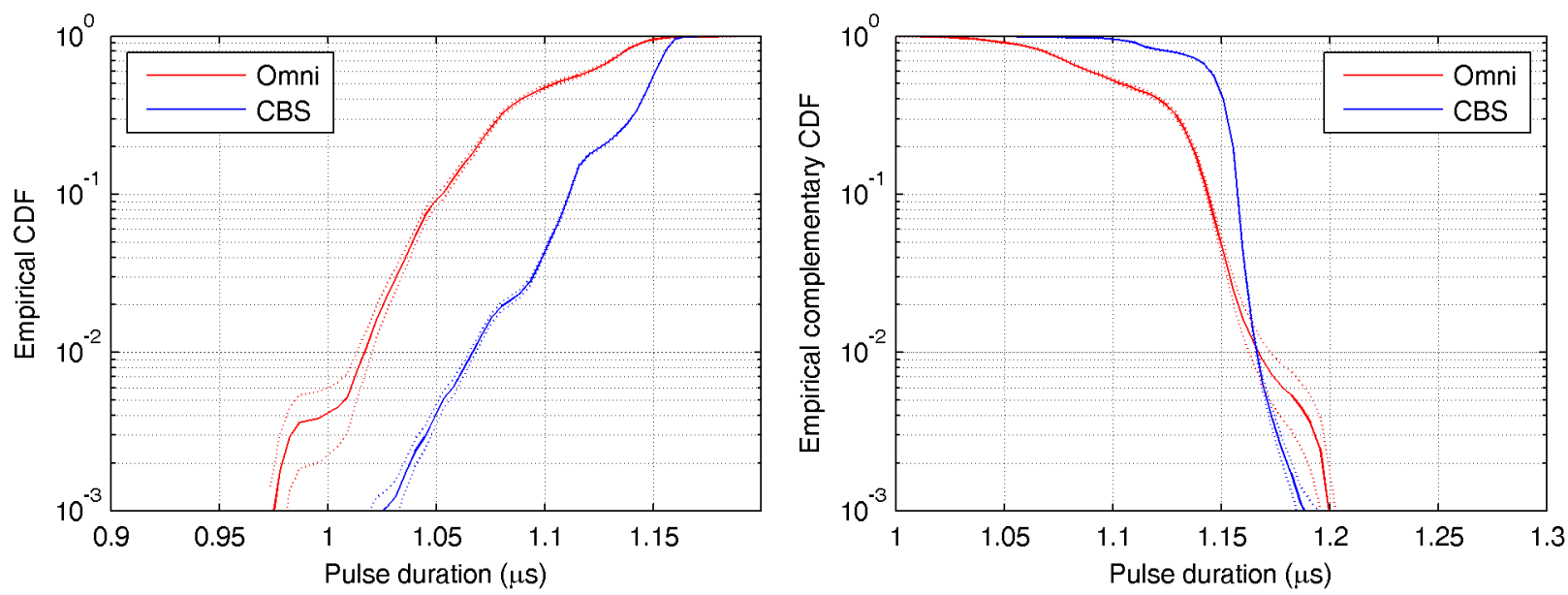

Figure 4.6: Empirical CDF and CCDF of pulse duration by antenna type
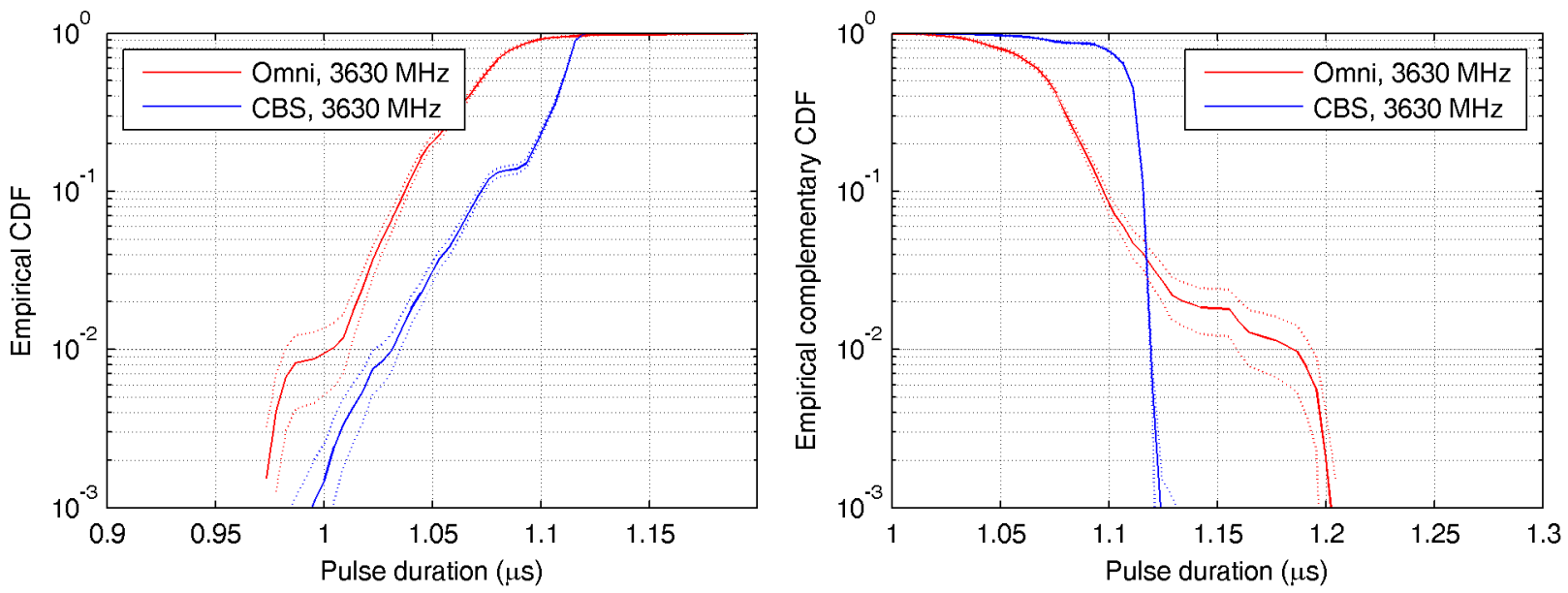

Figure 4.7: Empirical CDF and CCDF of pulse duration at $3630 \mathrm{MHz}$ by antenna type

quencies, with the vast majority of pulses observed at $3630 \mathrm{MHz}$ exhibiting noticeably shorter durations than those at the other two frequencies.

Fig. 4.6 shows the empirical CDF and CCDF of the pulse duration by the antenna used for data collection. We caution that the omnidirectional antenna data set is an order of magnitude smaller than the CBS antenna data set, which explains the larger confidence intervals around the distribution of the omnidirectional antenna data. Looking at the $3630 \mathrm{MHz}$ subset of the pulse duration data, which has a smaller disparity between the omnidirectional and CBS antenna data sizes, Fig. 4.7 shows the breakdown of that data by antenna. The $3630 \mathrm{MHz}$ pulse durations observed with an omnidirectional antenna exhibit a heavier upper tail than those observed with the CBS antenna. The greater frequency of longer pulse durations could be due to the longer paths of reflections and the different gain patterns of the antennas. An omnidirectional antenna receives 


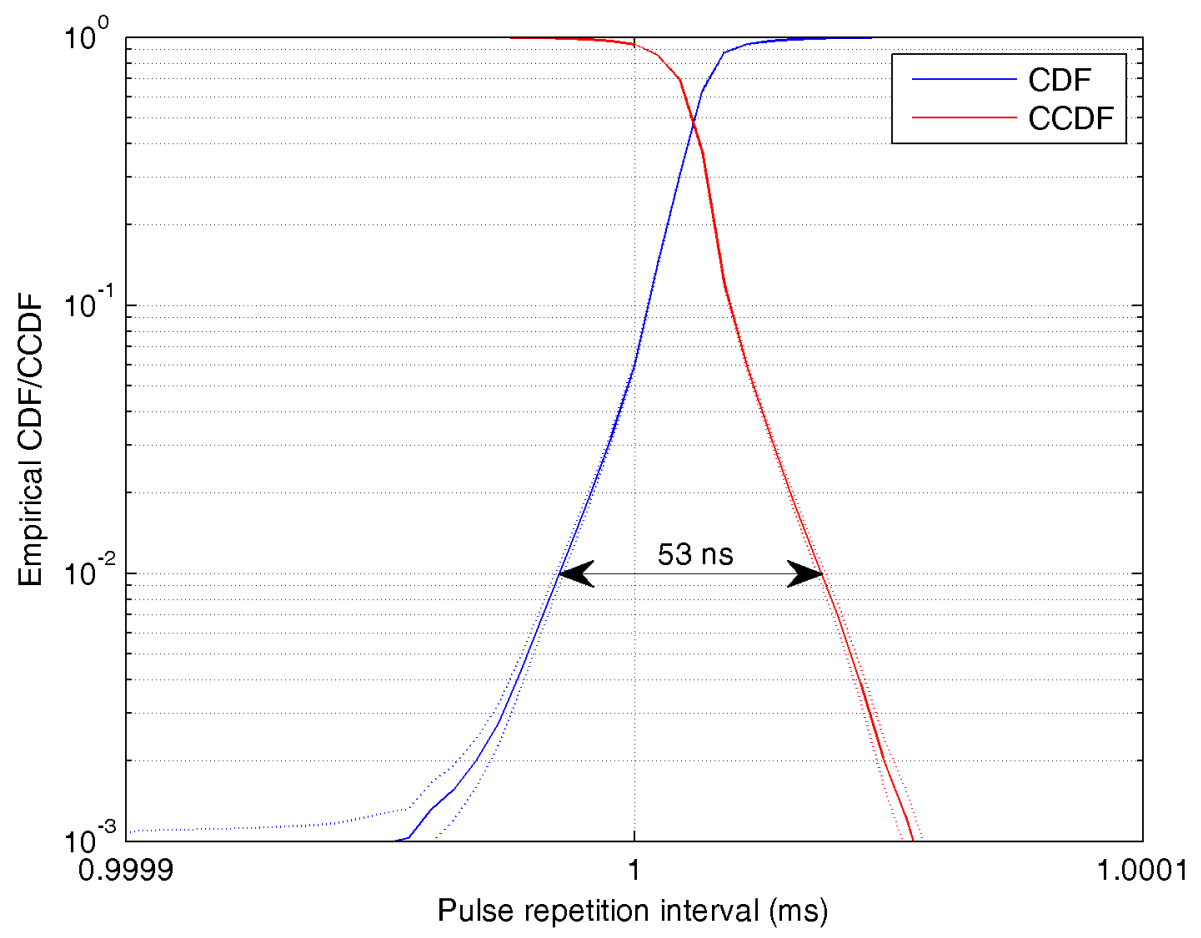

Figure 4.8: Empirical CDF and CCDF of pulse repetition interval over all data

pulse reflections off terrain and other objects from all azimuth angles with equal gain, whereas the CBS antenna has higher gain toward sea and lower gain toward potential reflectors on land. Other possible explanations include different ship positions or different atmospheric conditions between the two data sets.

\subsubsection{Pulse repetition interval}

The pulse repetition interval is defined as the time between the rising-edge crossings by the envelopes of two successive pulses of their respective half-amplitude levels. Fig. 4.8 plots the empirical CDF and CCDF of the pulse repetition interval aggregated over the total population of pulses. These results show a median pulse repetition interval that is within $13 \mathrm{~ns}$ of $1 \mathrm{~ms}$, and that $98 \%$ of the values lie within a range of $53 \mathrm{~ns}$.

Segmenting by antenna and center frequency, shown in Fig. 4.9, reveals no major differences between the data subsets except for heavier lower tails in the data obtained with the omnidirectional antenna at $3630 \mathrm{MHz}$. 

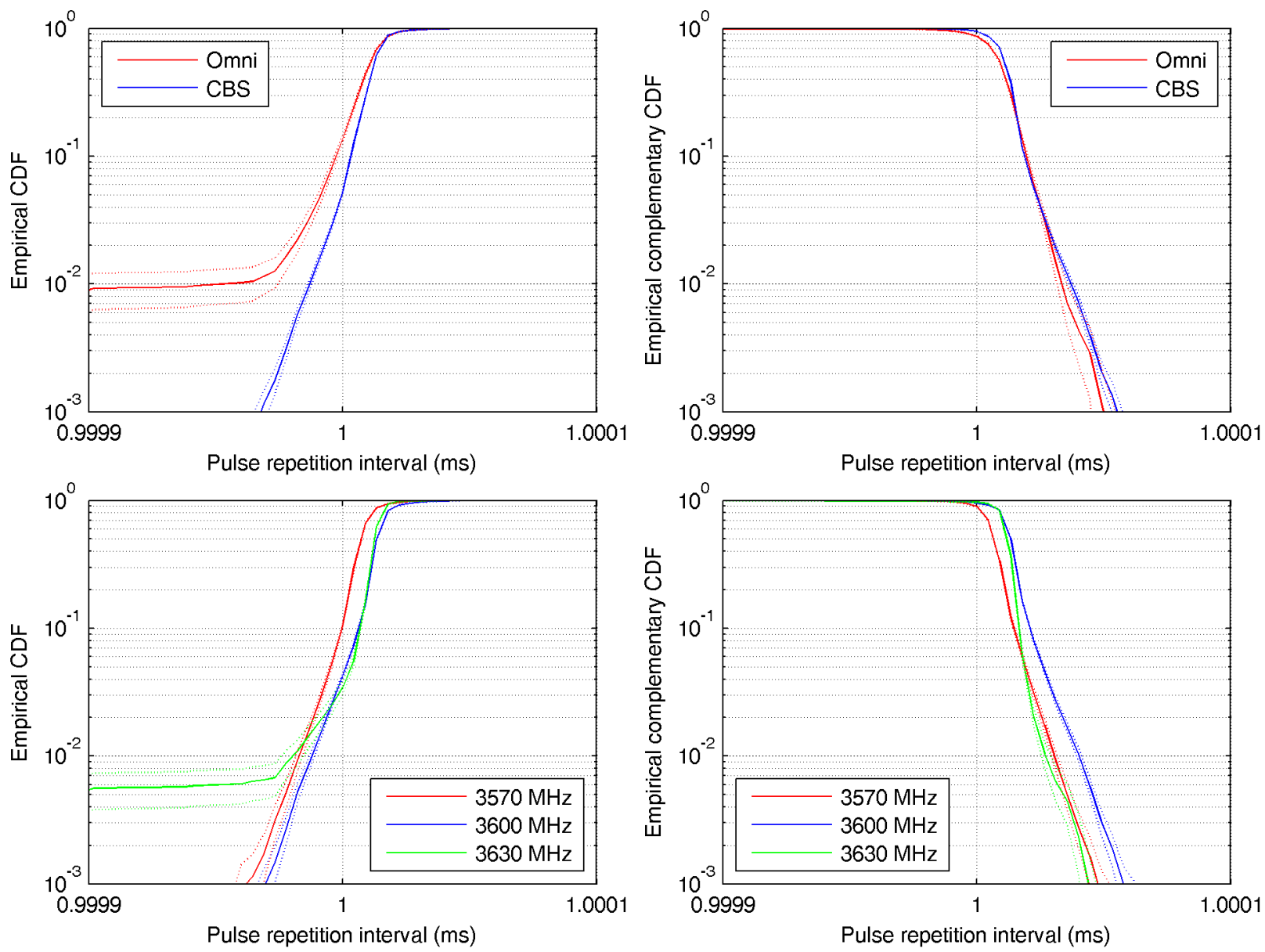

Figure 4.9: Empirical CDF and CCDF of pulse repetition interval by antenna type and by frequency 


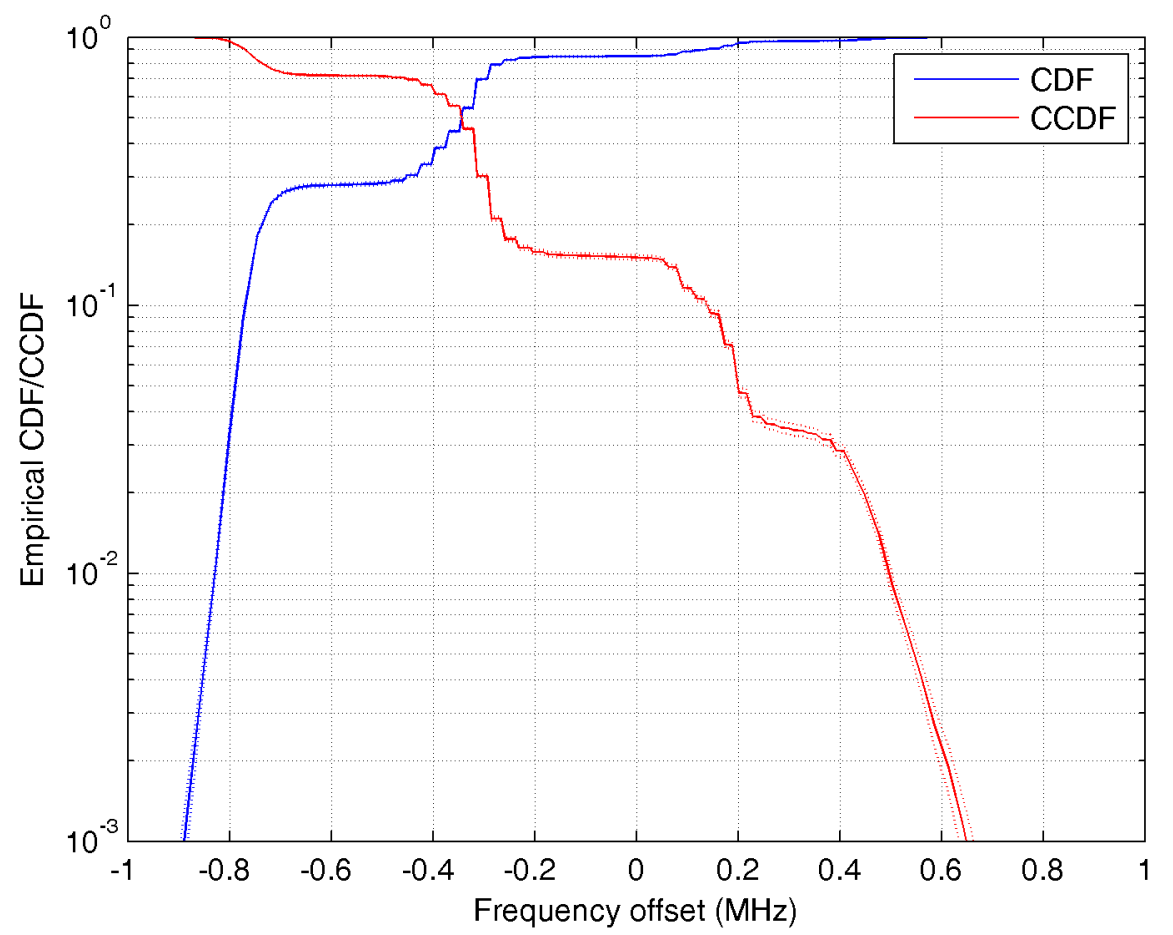

Figure 4.10: Empirical CDF and CCDF of frequency offset over all data

\subsubsection{Frequency offset}

The frequency offset is defined as the difference between the measured center frequency of a pulse and its nominal center frequency. ${ }^{4}$ Fig. 4.10 plots the empirical CDF and CCDF of the frequency offset aggregated over the total population of pulses. The empirical distribution shows a median offset of $-341 \mathrm{kHz}$ and 1st and 99th percentiles of $-828 \mathrm{kHz}$ and $503 \mathrm{kHz}$, respectively.

Fig. 4.11 plots the empirical distributions of the frequency offset by center frequency. The results show a larger spread in frequency offset for the $3570 \mathrm{MHz}$ and $3630 \mathrm{MHz}$ observations than for the $3600 \mathrm{MHz}$ data. Between the 1st and 99th percentiles, the offsets at $3570 \mathrm{MHz}$ and at $3630 \mathrm{MHz}$ vary by nearly one megahertz, but the $3600 \mathrm{MHz}$ offsets vary by $275 \mathrm{kHz}$. Fig. 4.12 shows a histogram of the same data which clearly shows the multimodal nature of the $3570 \mathrm{MHz}$ and $3630 \mathrm{MHz}$ data, in contrast to the apparent single mode of the $3600 \mathrm{MHz}$ data.

\footnotetext{
${ }^{4}$ The center frequency of a pulse is measured with an 8192-point FFT and, hence, has a resolution of $f_{S} / 8192=$ $225 \times 10^{6} / 8192 \mathrm{~Hz} \approx 27.5 \mathrm{kHz}$.
} 

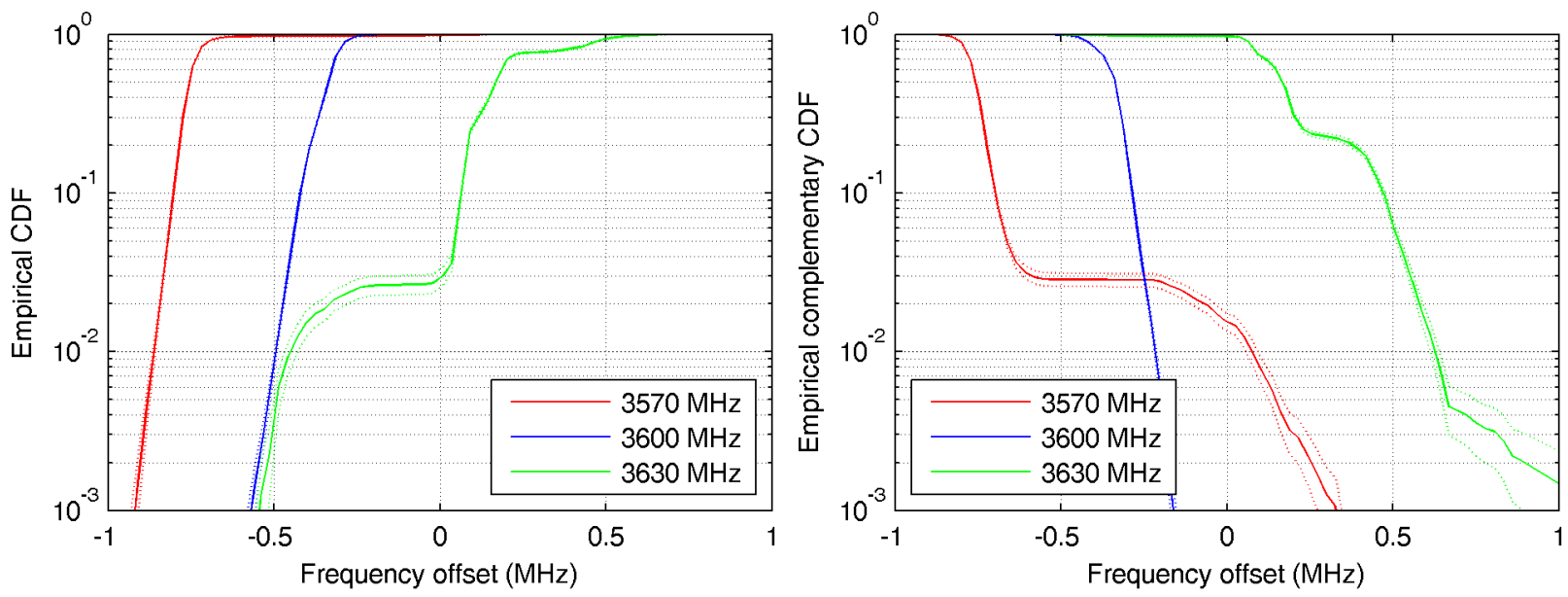

Figure 4.11: Empirical CDF and CCDF of frequency offset by center frequency

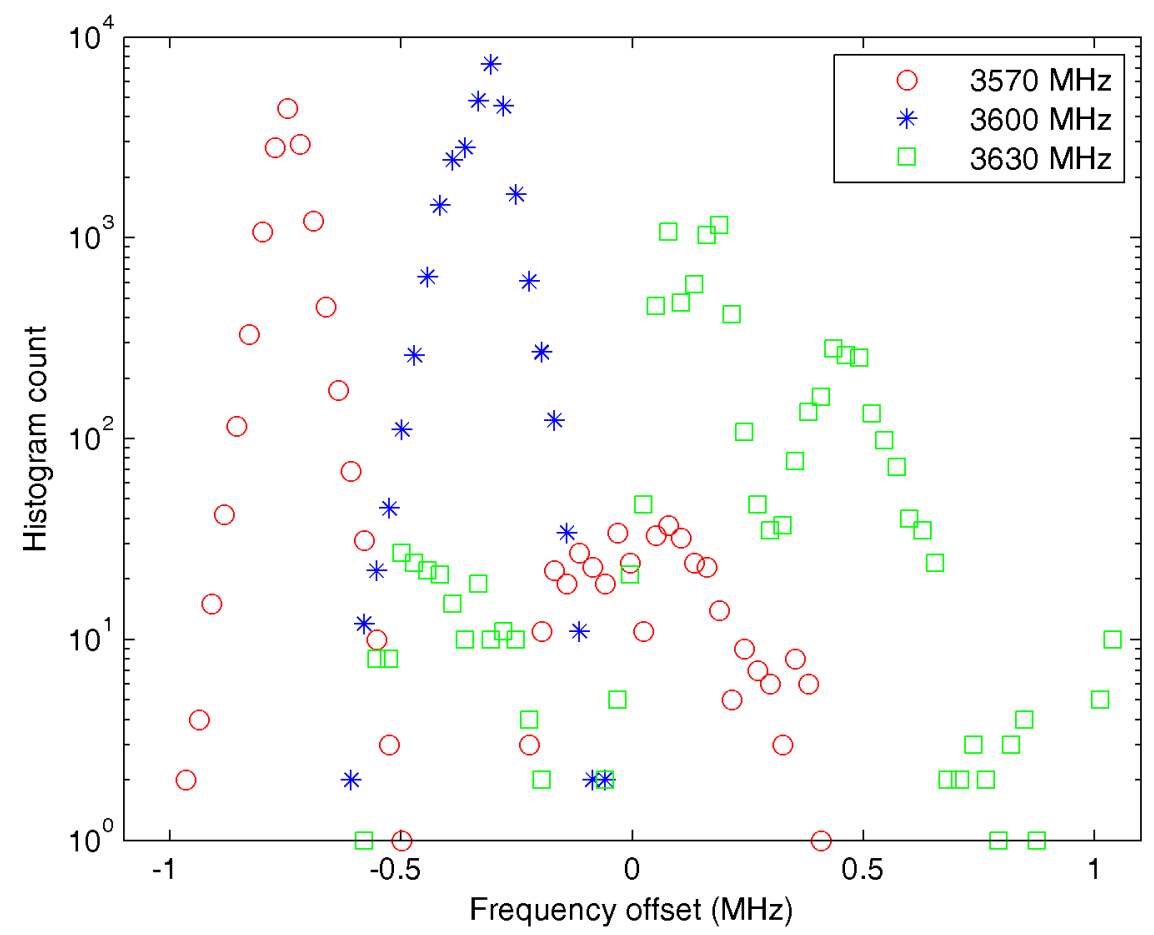

Figure 4.12: Histogram of frequency offset by center frequency 


\subsection{Summary statistics}

Table 4.3 summarizes the mean, median, and 1st and 99th percentiles of sweep and pulse parameters by antenna. Table 4.4 summarizes the statistics of pulse parameters by frequency. 


\begin{tabular}{|c|c|c|c|c|c|c|c|c|c|c|c|c|}
\hline & \multicolumn{4}{|c|}{ Omni } & \multicolumn{4}{|c|}{ CBS } & \multicolumn{4}{|c|}{ Overall } \\
\hline & Mean & Med & $1 \%$ & $99 \%$ & Mean & Med & $1 \%$ & $99 \%$ & Mean & Med & $1 \%$ & $99 \%$ \\
\hline Sweep Interval (s) & 4.347 & 3.858 & 3.725 & 13.638 & 4.409 & 3.858 & 3.837 & 19.275 & 4.402 & 3.858 & 3.834 & 18.727 \\
\hline Pulse Duration $(\mu \mathrm{s})$ & 1.104 & 1.107 & 1.018 & 1.169 & 1.143 & 1.151 & 1.067 & 1.169 & 1.140 & 1.151 & 1.049 & 1.169 \\
\hline
\end{tabular}

Table 4.3: Statistics of sweep and pulse parameters by antenna: mean, median, 1 st percentile (1\%), and 99 th percentile (99\%)

\begin{tabular}{|c|c|c|c|c|c|c|c|c|c|c|c|c|}
\hline & \multicolumn{4}{|c|}{$3570 \mathrm{MHz}$} & \multicolumn{4}{|c|}{$3600 \mathrm{MHz}$} & \multicolumn{4}{|c|}{$3630 \mathrm{MHz}$} \\
\hline & Mean & Med & $1 \%$ & $99 \%$ & Mean & Med & $1 \%$ & $99 \%$ & Mean & Med & $1 \%$ & $99 \%$ \\
\hline Sweep Interval (s) & 4.313 & 3.882 & 3.857 & 15.551 & 4.566 & 3.857 & 3.843 & 22.240 & 4.030 & 3.856 & 3.738 & 7.472 \\
\hline Pulse Duration $(\mu \mathrm{s})$ & 1.142 & 1.147 & 1.080 & 1.164 & 1.150 & 1.156 & 1.098 & 1.169 & 1.096 & 1.107 & 1.018 & 1.124 \\
\hline Pulse Repet. Intrvl. - 1 ms (ns) & 9 & 9 & -13 & 31 & 16 & 18 & -9 & 44 & 13 & 13 & -18 & 31 \\
\hline Frequency Offset (kHz) & -721 & -745 & -855 & 79 & -332 & -313 & -478 & -203 & 213 & 174 & -430 & 641 \\
\hline
\end{tabular}

Table 4.4: Statistics of sweep and pulse parameters by center frequency 


\section{Chapter 5}

\section{Conclusions}

The Fort Story measurement campaign resulted in a total of 617960 -s power spectra and 1473 highresolution 60-s waveforms. Over the course of these measurements, the target SPN-43 radar system was observed at three different operational frequencies: $3570 \mathrm{MHz}, 3600 \mathrm{MHz}$, and $3630 \mathrm{MHz}$. In addition to observing SPN-43 at these frequencies in "normal" operation, the following phenomena were observed and are documented in this report:

- Tuning of the radar across a contiguous range of frequencies

- Adjacent-band emissions into the band of interest

- Simultaneous emission from two SPN-43 systems in two different frequency channels

Furthermore, the adjacent-band emissions were frequently observed to be coincident and cochannel with SPN-43 emissions, indicating that the adjacent-band emissions could confound SPN43 detection by an ESC.

A subset of the high-resolution IQ waveforms containing no adjacent-band or other in-band emissions was selected for further statistical analysis. This analysis focused on key parameters of the target radar that could aid detection, namely, the antenna rotation period, pulse repetition interval, pulse duration, and center frequency. The subset of approximately 250 "clean" acquisitions of in-band radar consisted of 2700 sweeps of the main beam and 48600 individual pulses ranging in peak power by over $40 \mathrm{~dB}$. The statistical analysis of in-band radar parameters yielded the following observations.

- The median antenna rotation period was $3.86 \mathrm{~s}, 0.14 \mathrm{~s}$ lower than reported in the SPN43 system manual dated 2005 [2]. The difference between the 10th and 90th percentiles (containing $80 \%$ of the observations) was $0.06 \mathrm{~s}$.

- The median pulse repetition interval was very close to the nominal value of $1 \mathrm{~ms}$ (only $13 \mathrm{~ns}$ 
greater). Furthermore, the difference between the 1st and 99th percentiles (containing $98 \%$ of the values) was only $53 \mathrm{~ns}$.

- The overall median pulse duration was $1.15 \mu \mathrm{s}$ and varied by $0.05 \mu \mathrm{s}$ across the three observed frequencies. The 1st and 99th percentiles of the pulse duration were $1.05 \mu \mathrm{s}$ and $1.17 \mu \mathrm{s}$, respectively. The median pulse duration observed here is $0.20 \mu \mathrm{s}$ longer than that reported in the SPN-43 system manual [2].

- The median frequency offset from the nominal center frequency ranged from $174 \mathrm{kHz}$ to $745 \mathrm{kHz}$ across the three observed frequencies, and their $98 \%$ spreads varied from $275 \mathrm{kHz}$ to $1.07 \mathrm{MHz}$.

Knowledge of these statistics can be used to develop robust ESC detectors. They can also inform the development and selection of waveforms with which these detectors can be tested by a certification body. Furthermore, the waveforms themselves could be "played back"-that is, converted to an analog signal and upconverted to the appropriate radio frequency (RF) - by a vector signal generator to evaluate the performance of an ESC detector. A benefit of playing back the measured waveforms is that the effects of the propagation channel, which can distort the signal, are inherent in these waveforms. Hence, the measured waveforms better reflect the actual signals an ESC will receive in the field.

It should be emphasized that our measurements at Fort Story were observational, with many uncontrolled variables (see Section 2.2.2), and were limited to a single geographic site and time interval. Consequently, the findings reported here should not be used to make overly general inferences about SPN-43 emissions that may be seen in the field. Additionally, care should be taken when comparing the descriptive statistics for our measurements at Fort Story to those at Point Loma [1], since many factors differed between the two sites. 


\section{Chapter 6}

\section{Areas for Future Research}

A potential near-term use of the $3.5 \mathrm{GHz}$ waveforms collected in this study is for controlled testing of CBRS ESC systems. At a minimum, the analysis of pulse and sweep parameters provided in Chapter 4 can inform the parametrization of surrogate waveforms being developed by NTIA/ITS for laboratory testing of ESC detectors.

Furthermore, the waveforms themselves could be "played back" - that is, converted to an analog signal and upconverted to the appropriate RF frequency - by a vector signal generator to evaluate the performance of an ESC detector. A benefit of playing back the measured waveforms is that the effects of the propagation channel, which can distort the signal, are inherent in these waveforms. Hence, the measured waveforms better reflect the actual signals an ESC will receive in the field. Future work could determine an appropriate subset of the collected waveforms that represents the range of parameters, channel conditions, and signal-to-noise ratios that were observed in the field.

Waveform recordings and pulse parameters at lower power and SNR might be used to better inform the use of surrogate waveforms or waveform play back. Future work could use equipment with a lower noise figure and focus on measuring signals at a lower trigger threshold. Alternatively, the existing measurements might be better explored with more robust pulse detection and parameter estimation algorithms or algorithms that are more informative about the pulse shape, such as second and higher order moments of the pulse delay spread.

The primary federal incumbent radar system in $3550 \mathrm{MHz}$ to $3650 \mathrm{MHz}$ is the SPN-43 air traffic control radar [10], and the emissions of that system were the focus of this study. However, future Navy maritime radars are under development that will also occupy this band, and their emissions will need to be detected by an ESC as well. Since the waveforms of these future radars are classified, an important area of future research is the development of surrogate waveforms for 
these radars that can be used for the design, development, and testing of ESC detectors.

The research and development of surrogate waveforms for future radars should encompass the validation of the surrogate waveforms against real emissions of these radars. Such a validation could include testing detection algorithms with both the surrogates and the real signals to ensure they elicit comparable detection performance across a range of operating conditions.

Finally, research into channel models and their validation for the $3.5 \mathrm{GHz}$ band would be valuable on two fronts. First, measurements of propagation loss across a wide range of transmitterreceiver locations, antenna heights, and clutter environments would help to validate existing and future models. These models are being used to develop static CBRS exclusion and protection zones and will be used by a CBRS Spectrum Access System for dynamic aggregate interference computations.

Second, wideband channel models that include the effects of the channel on pulse shape could be used in conjunction with the surrogate waveforms to render them more realistic. Convolving idealized radar signals with a time-varying channel impulse response derived from field measurements would emulate the dispersive and fading channels that will be encountered by ESC detectors in the field. Hence, the wideband channel models derived from this research would potentially improve the fidelity of tests employing surrogate waveforms. 


\section{Bibliography}

[1] P. Hale, J. Jargon, P. Jeavons, M. Lofquist, M. Souryal, and A. Wunderlich, "3.5 GHz radar waveform capture at Point Loma," NIST TN 1954, Report 2, National Advanced Spectrum and Communications Test Network, Apr. 2017.

[2] "Operation and maintenance instructions, organizational level, radar set AN/SPN-43C." Naval Air Systems Command, Technical Manual, EE216-EB-OMI-010, vol. 1, Sept. 2005.

[3] "Amendment of the commission's rules with regard to commercial operations in the 3550$3650 \mathrm{MHz}$ band." FCC Report and Order and Second Further Notice of Proposed Rulemaking, GN Docket No. 12-354, Apr. 2015.

[4] "Citizens broadband radio service." 2 C.F.R. $\S$ 96, 2016.

[5] Military Standardization Handbook. Department of Defense, MIL-HDBK-162B, Dec. 1973.

[6] M. G. Cotton and R. A. Dalke, "Spectrum occupancy measurements of the 3550-3650 megahertz maritime radar band near San Diego, California," Technical Report TR 14-500, National Telecommunications and Information Administration, Jan. 2014.

[7] "Government briefing on AN/SPN-43C radar set capabilities/interfaces." Wireless Innovation Forum Document WINNF-16-I-0105, Jan. 2016.

[8] F. H. Sanders, J. E. Carroll, G. A. Sanders, and L. S. Cohen, "Measurements of selected naval radar emissions for electromagnetic compatibility analyses," Technical Report TR 15510, National Telecommunications and Information Administration, Oct. 2014.

[9] "Requirements for commercial operation in the U.S. 3550-3700 MHz citizens broadband radio service band." Wireless Innovation Forum Document WINNF-15-S-0112, Version V2.0.0, Feb. 2017.

[10] "An assessment of the near-term viability of accommodating wireless broadband systems in the 1675-1710 MHz, 1755-1780 MHz, 3500-3650 MHz, 4200-4220 MHz and 4380-4400 MHz bands," tech. rep., U.S. Department of Commerce, National Telecommunications and 
Information Administration, Oct. 2010.

[11] J. A. Jargon, D. F. Williams, and P. D. Hale, "Developing models for type-N coaxial VNA calibration kits within the NIST microwave uncertainty framework," in Proc. 87th ARFTG Microwave Measurement Conference, (San Francisco, CA), May 2016.

[12] I. BIPM, IEC, IFCC, ILAC, ISO, IUPAC and OIML, Evaluation of measurement data: Guide to the expression of uncertainty in measurement. Joint Committee for Guides in Metrology JCGM 100:2008, Sept. 2008.

[13] T. E. Parker, S. R. Jefferts, and T. P. Heavner, "Medium-term frequency stability of hydrogen masers as measured by a cesium fountain," in 2010 IEEE International Frequency Control Symposium, pp. 318-323, June 2010.

[14] R. J. Matheson, J. D. Smilley, G. D. Falcon, and V. S. Lawrence, “Output tube emission characteristics of operational radars," Technical Report TR 82-92, National Telecommunications and Information Administration, Jan. 1982.

[15] T. E. Ruden, "Design and performance of a one megawatt, 3.1-3.5 GHz coaxial magnetron," in 1979 9th European Microwave Conference, pp. 731-735, Sept. 1979.

[16] "Electromagnetic compatibility criteria for navy systems." NAVSEA S9407-AA GYD-010/S op-3840 Rev 5, classified SECRET, Jan. 2016.

[17] W. N. Venables and B. D. Ripley, Modern Applied Statistics with S. New York: Springer, 4th ed., 2013. 